Supporting Information

\title{
Activation of Ene-Diamido Samarium Methoxide with Hydrosilane for Selectively Catalytic Hydrosilylation of Alkenes and Polymerization of Styrene: an Experimental and Theoretical Mechanistic Study
}

Jianfeng Li, ${ }^{+}$Chaoyue Zhao,${ }^{\dagger}$ Jinxi Liu, ${ }^{+}$Hanmin Huang, ${ }^{+}$Fengxin Wang, ${ }^{+}$Xiufang $\mathrm{Xu}^{*,+}$ and Chunming $\mathrm{Cui}^{*,+, *}$

${ }^{+}$State Key Laboratory of Elemento-Organic Chemistry, Nankai University, Tianjin 300071 ; ${ }^{\ddagger}$ Collaborative Innovation Center of Chemical Science and Engineering (Tianjin), Tianjin 300071, P. R. China

E-mail: cmcui@nankai.edu.cn xxfang@nankai.edu.cn

Homepage: http://cui.nankai.edu.cn 


\section{Table of Contents}

$\begin{array}{lr}\text { General Experimental } & \mathbf{S 3}\end{array}$

Kinetic Studies $\quad$ S6

$\begin{array}{lr}\text { X-ray Diffraction Parameters and Data } & \text { S8 }\end{array}$

$\begin{array}{ll}\text { Computational Details } & \text { S9 }\end{array}$

$\begin{array}{lr}\text { Reference } & \text { S70 }\end{array}$

$\begin{array}{ll}\text { NMR Spectra of } \mathrm{LK}_{2} \text { and } 1 & \text { S71 }\end{array}$

$\begin{array}{ll}\text { NMR Spectra of Hydrosilylation Product } & \text { S73 }\end{array}$

$\begin{array}{ll}\text { Spectra of } s P S & \mathbf{S 7 5}\end{array}$

$\begin{array}{lr}\text { NMR Spectra of Crude Hydrosilylation Reaction Mixtures } & \text { S78 }\end{array}$

GC-MS Spectra of Crude Hydrosilylation Reaction Mixtures $\quad$ S83 


\section{General Experimental}

All manipulations involving air- and moisture-sensitive compounds were carried out under an atmosphere of dry argon by using modified Schlenk line and glovebox techniques. Elemental analyses were carried out on an Elementar Vario EL analyzer. EI-MS were recorded on VG ZAB-HS. The ${ }^{1} \mathrm{H},{ }^{13} \mathrm{C}$, and ${ }^{29} \mathrm{Si}$ NMR spectroscopic data were recorded on a Bruker AV400 spectrometer. GC-MS data were obtained with a Thermo Scientific TRACE 1300 \& ISQ QD system, equipped with a TG-5MS column. Infrared spectra were recorded on a Bio-Rad FTS 6000 spectrophotometer. Gel permeation chromatography (GPC) analyses of the molecular weight and molecular weight distribution were performed on a Waters 1525 instrument using standard polystyrene as calibration and with THF as the eluent at $25^{\circ} \mathrm{C}$.

The solvents (DME, THF, toluene, and $n$-hexane) were freshly distilled from sodium and degassed prior to use. $\alpha$-diimine, $\mathrm{LH}_{2}$ and $\mathrm{Sm}\left[\mathrm{N}\left(\mathrm{SiMe}_{3}\right)_{2}\right]_{2}(\mathrm{THF})_{2}$ were synthesized according to the published procedures. ${ }^{\mathrm{S} 1, \mathrm{~S} 2}$ Alkenes and dienes were vacuum-distilled over $\mathrm{CaH}_{2}$ and then were degassed prior to use. Other chemicals were of analytical grade and were used as received.

Synthesis of $\mathbf{L K}_{2}$. A mixture of potassium $(160 \mathrm{mg}, 4 \mathrm{mmol})$ and $\alpha$-diimine (808 $\left.\mathrm{mg}, 2 \mathrm{mmol}\right)$ in DME $(30 \mathrm{~mL})$ was stirred at room temperature for 3 days. The volatiles were removed under vaccum. The remaining residue was washed by $n$-hexane and subsequently dried under vaccum to yield red powder of $\mathrm{LK}_{2}(996 \mathrm{mg}, 87 \%) . \mathrm{Mp}: 231{ }^{\circ} \mathrm{C}$ dec. ${ }^{1} \mathrm{H}$ NMR (THF- $\left.d 8,400 \mathrm{MHz}, 23{ }^{\circ} \mathrm{C}\right): \delta 1.03\left(\mathrm{~d},{ }^{3} J_{\mathrm{HH}}=6.9 \mathrm{~Hz}, 12 \mathrm{H}\right.$, $\mathrm{CHMe} 2), 1.21\left(\mathrm{~d},{ }^{3} \mathrm{~J}_{\mathrm{HH}}=6.8 \mathrm{~Hz}, 12 \mathrm{H}, \mathrm{CHMe}\right), 1.66(\mathrm{~s}, 6 \mathrm{H}, \mathrm{CMe}), 3.67\left(\mathrm{sept},{ }^{3} \mathrm{~J}_{\mathrm{HH}}=6.8 \mathrm{~Hz}, 4 \mathrm{H}, \mathrm{CHMe} 2\right)$, $5.97\left(\mathrm{t},{ }^{3} J_{\mathrm{HH}}=7.3 \mathrm{~Hz}, 2 \mathrm{H}, \mathrm{Ar}-H\right), 6.63\left(\mathrm{~d},{ }^{3} \mathrm{~J}_{\mathrm{HH}}=7.3 \mathrm{~Hz}, 4 \mathrm{H}, \mathrm{Ar}-H\right) .{ }^{13} \mathrm{C}$ NMR $\left(\mathrm{THF}-d_{8}, 101 \mathrm{MHz}, 23{ }^{\circ} \mathrm{C}\right)$ : $\delta 18.95$ (CHMe2), 24.45 (CMe), 27.68 (CHMe 2$), 59.06,72.82$ (DME-C), 109.31 (CMe), 121.47, 123.09, $137.37,155.80(\operatorname{Ar}-C)$.

Synthesis of 1. Amine Elimination Method: A mixture of $\mathrm{Sm}\left[\mathrm{N}\left(\mathrm{SiMe}_{3}\right)_{2}\right]_{2}(\mathrm{THF})_{2}(1.230 \mathrm{~g}, 2 \mathrm{mmol})$ and $\mathrm{LH}_{2}(812 \mathrm{mg}, 2 \mathrm{mmol})$ was added $30 \mathrm{~mL}$ DME. The solution was stirred at room temperature for $20 \mathrm{~h}$. The solution was concentrated to $5 \mathrm{~mL}$. Storage at $-35{ }^{\circ} \mathrm{C}$ overnight afforded brown crystals of 1 (613 $\mathrm{mg}$, 46\%). Salt Metathesis Method: A solution of $\mathrm{SmI}_{2}(\mathrm{THF})_{2}(1.096 \mathrm{~g}, 2 \mathrm{mmol})$ in DME was added freshly 
prepared $\mathrm{LK}_{2}(2 \mathrm{mmol})$ in DME. The solution was stirred at room temperature for $20 \mathrm{~h}$. After removing the solvent under reduced pressure, the residue was extracted by toluene. The filtrate was concentrated to $5 \mathrm{~mL}$. Storage at $-35{ }^{\circ} \mathrm{C}$ overnight afforded brown crystals of 1 (1.137 g, 84\%). Mp: 232-234 ${ }^{\circ} \mathrm{C}$. Anal. Calcd for $\mathrm{C}_{66} \mathrm{H}_{106} \mathrm{~N}_{4} \mathrm{O}_{6} \mathrm{Sm}_{2}$ (1352.29): C, 58.62; H, 7.90; N, 4.14. Found: C, 58.34; H, 7.60; N, 4.53. 1 is paramagnetic. ${ }^{1} \mathrm{H}$ NMR $\left(\mathrm{C}_{6} \mathrm{D}_{6}, 400 \mathrm{MHz}, 23{ }^{\circ} \mathrm{C}\right): \delta-3.56(\mathrm{~s}, 8 \mathrm{H}, \mathrm{DME}-H),-1.70$ (s, 12H, DME-H), -1.59 (s, 12H, DME-H), $-0.49(\mathrm{~s}, 8 \mathrm{H}, \mathrm{DME}-H), 1.34\left(\mathrm{~d},{ }^{3} J_{\mathrm{HH}}=6.5 \mathrm{~Hz}, 12 \mathrm{H}, \mathrm{CHMe}\right), 2.29(\mathrm{~s}, 12 \mathrm{H}, \mathrm{CMe}) 3.03\left(\mathrm{~d},{ }^{3} J_{\mathrm{HH}}=\right.$ $6.4 \mathrm{~Hz}, 12 \mathrm{H}, \mathrm{CHMe}), 3.29\left(\mathrm{~d},{ }^{3} J_{\mathrm{HH}}=6.2 \mathrm{~Hz}, 12 \mathrm{H}, \mathrm{CHMe} 2\right), 3.95\left(\mathrm{~d},{ }^{3} J_{\mathrm{HH}}=6.3 \mathrm{~Hz}, 12 \mathrm{H}, \mathrm{CHMe}\right), 4.44(\mathrm{~s}$, $6 \mathrm{H}, \mathrm{OMe}), 5.17\left(\mathrm{~d},{ }^{3} J_{\mathrm{HH}}=7.5 \mathrm{~Hz}, 2 \mathrm{H}, \mathrm{Ar}-H\right), 6.12\left(\mathrm{t},{ }^{3} J_{\mathrm{HH}}=7.4 \mathrm{~Hz}, 4 \mathrm{H}, \mathrm{Ar}-H\right), 6.35\left(\mathrm{~d},{ }^{3} J_{\mathrm{HH}}=7.5 \mathrm{~Hz}, 2 \mathrm{H}\right.$, $\operatorname{Ar}-H), 7.34\left(\mathrm{t},{ }^{3} J_{\mathrm{HH}}=7.5 \mathrm{~Hz}, 2 \mathrm{H}, \mathrm{Ar}-H\right), 7.97\left(\mathrm{~d},{ }^{3} J_{\mathrm{HH}}=7.6 \mathrm{~Hz}, 2 \mathrm{H}, \mathrm{Ar}-H\right), 9.25\left(\mathrm{sept},{ }^{3} J_{\mathrm{HH}}=6.7 \mathrm{~Hz}, 4 \mathrm{H}\right.$, $\mathrm{CHMe} 2), 11.57\left(\mathrm{sept},{ }^{3} \mathrm{H}_{\mathrm{HH}}=6.6 \mathrm{~Hz}, 4 \mathrm{H}, \mathrm{CHMe} 2\right) .{ }^{13} \mathrm{C} \mathrm{NMR}\left(\mathrm{THF}-d_{8}, 101 \mathrm{MHz}, 23{ }^{\circ} \mathrm{C}\right): \delta 14.60,18.96$, $20.94,21.86,22.31,24.43,25.47,26.67,27.23,27.55,29.06,29.20,29.54,30.08,31.03,32.57,59.06,67.57$, $72.84,117.07,118.37,119.93,121.56,121.86,124.25,129.82,130.90,131.42,131.74,131.88,137.44$, $150.99,152.81$.

Typical Procedure for Catalytic Hydrosilylation. In an Ar glove box, a Schlenk tube was charged with catalyst $(2.5 \mathrm{~mol} \%)$ and solvent $(1 \mathrm{~mL})$. And then $\mathrm{PhSiH}_{3}(1 \mathrm{mmol})$ and the appropriate alkene or diene (1 mmol) were added by microsyringe. The Schlenk tube was quickly removed from the glovebox. The reaction mixture was stirred at $60{ }^{\circ} \mathrm{C}$ using oil bath. The reaction was monitored by ${ }^{1} \mathrm{H}$ NMR spectroscopy. After the reaction was complete, the product was purified by column chromatography on silica gel ( $n$-hexane for elution) to yield colorless oil. The spectra of hydrosilylated products $\mathbf{3 a},{ }^{\mathrm{S} 3} \mathbf{3 c},{ }^{\mathrm{S} 3} \mathbf{3 d},{ }^{\mathrm{S} 4} \mathbf{3 e},{ }^{\mathrm{S} 5}$ $\mathbf{3 f},{ }^{\mathrm{S} 3} \mathbf{3 g},{ }^{\mathrm{S} 3} \mathbf{3 h},{ }^{\mathrm{S} 3}$ and $\mathbf{3 i}{ }^{\mathrm{S} 6}$ were consistent with literature. $\mathbf{3 b}\left(210 \mathrm{mg}, 96 \%\right.$ of yield): ${ }^{1} \mathrm{H}$ NMR $\left(\mathrm{C}_{6} \mathrm{D}_{6}, 400\right.$ $\left.\mathrm{MHz}, 23{ }^{\circ} \mathrm{C}\right): \delta 1.36\left(\mathrm{~d},{ }^{3} J_{\mathrm{HH}}=7.5 \mathrm{~Hz}, 3 \mathrm{H}, \mathrm{CHMe}\right), 2.12(\mathrm{~s}, 3 \mathrm{H}, \mathrm{Ar}-M e), 2.43\left(\mathrm{~m}, 1 \mathrm{H}, \mathrm{SiH}_{2} \mathrm{CH}\right), 4.51(\mathrm{~m}$, $\left.\mathrm{Si} H_{2}\right), 6.97-7.38(\mathrm{~m}, 9 \mathrm{H}, \mathrm{Ar}-H) .{ }^{13} \mathrm{C} \mathrm{NMR}\left(\mathrm{C}_{6} \mathrm{D}_{6}, 101 \mathrm{MHz}, 23{ }^{\circ} \mathrm{C}\right): \delta 16.73(\mathrm{CHMe}), 21.00(\mathrm{Si}-\mathrm{C}), 25.19$ (Ar-Me) $127.44,128.18,129.49,130.01,131.83,134.55,136.04,141.64(\mathrm{Ar}-C) .{ }^{29} \mathrm{Si} \mathrm{NMR}\left(\mathrm{C}_{6} \mathrm{D}_{6}, 79 \mathrm{MHz}\right.$, $\left.23{ }^{\circ} \mathrm{C}\right): \delta-21.4 . \mathrm{EI}-\mathrm{MS}, \mathrm{m} / \mathrm{z} 226.23$. 
Regioselectivity were analysed by GC-MS of the crude reaction mixture, after quenching and diluting the crude reaction mixture with $n$-hexane.

Typical Procedure for Catalytic Polymerization. In an Ar glove box, a Schlenk tube was charged with catalyst $(0.0125 \mathrm{mmol})$ and toluene $(0.5 \mathrm{~mL})$. And then $\mathrm{PhSiH}_{3}(0.5 \mathrm{mmol})$ and styrene $(5 \mathrm{mmol})$ were added by microsyringe sequentially. The Schlenk tube was quickly removed from the glovebox. The reaction mixture was stirred at $60{ }^{\circ} \mathrm{C}$ using oil bath. The reaction was quenched by addition of methanol after $6 \mathrm{~h}$. The polymer was collected by filtration and then washed by methanol three times, followed by drying uder high vacuum. White solid (369 mg, 71\% of yield). The syndiotacticity is $>99 \%$ by ${ }^{1} \mathrm{H}$ and ${ }^{13} \mathrm{C}$ NMR. $M_{\mathrm{n}}=5100$, PDI $=1.33$ by GPC. ${ }^{1} \mathrm{H}$ NMR (relative intensity, $\left.\mathrm{CDCl}_{3}, 400 \mathrm{MHz}, 23{ }^{\circ} \mathrm{C}\right): \delta 0.95(2.3$, $\mathrm{CHMe}), 1.37\left(66, \mathrm{CH}_{2}\right), 1.82(33, \mathrm{CH}), 4.08$ (1.5, $\left.\mathrm{Si} H_{2}\right), 6.60$ (66, Ar- $\left.H\right), 7.11$ (100, Ar-H). ${ }^{13} \mathrm{C}$ NMR $\left(\mathrm{CDCl}_{3}, 101 \mathrm{MHz}, 23{ }^{\circ} \mathrm{C}\right): \delta 23.8,40.7,44.0,125.8,127.8,128.0,145.4 .{ }^{29} \mathrm{Si} \mathrm{NMR}\left(\mathrm{CDCl}_{3}, 79 \mathrm{MHz}\right.$, $\left.23{ }^{\circ} \mathrm{C}\right): \delta-21.8 . \mathrm{IR}\left(\mathrm{cm}^{-1}\right): \tilde{v} 2130(\mathrm{Si}-\mathrm{H})$. 
Kinetic Studies

Table S1. Measurement of the 1-hexene concentration in different catalyst concentrations during the hydrosilylation process. ${ }^{[\mathrm{a}]}$

\begin{tabular}{|c|c|c|c|c|}
\hline entry & [catalyst] (M) & time $(\min )$ & $\ln \left([\right.$ hexene $\left.] /[\text { hexene }]_{0}\right)$ & $10^{5} \times k_{\mathrm{obs}}\left(\mathrm{s}^{-1}\right)$ \\
\hline 1 & \multirow{5}{*}{0.0148} & 37 & -0.16605 & \multirow{5}{*}{8.833} \\
\hline 2 & & 96 & -0.46681 & \\
\hline 3 & & 170 & -0.90011 & \\
\hline 4 & & 247 & -1.26585 & \\
\hline 5 & & 354 & -1.92415 & \\
\hline 6 & \multirow{5}{*}{0.0178} & 34 & -0.19845 & \multirow{5}{*}{10.417} \\
\hline 7 & & 92 & -0.52256 & \\
\hline 8 & & 172 & -1.07275 & \\
\hline 9 & & 252 & -1.53712 & \\
\hline 10 & & 358 & -2.28278 & \\
\hline 11 & \multirow{5}{*}{0.0251} & 31 & -0.25231 & \multirow{5}{*}{15.300} \\
\hline 12 & & 83 & -0.76411 & \\
\hline 13 & & 139 & -1.26942 & \\
\hline 14 & & 191 & -1.75751 & \\
\hline 15 & & 244 & -2.24432 & \\
\hline 16 & \multirow{5}{*}{0.0296} & 28 & -0.26788 & \multirow{5}{*}{17.667} \\
\hline 17 & & 85 & -0.88916 & \\
\hline 18 & & 131 & -1.34947 & \\
\hline 19 & & 177 & -1.89699 & \\
\hline 20 & & 247 & -2.63109 & \\
\hline
\end{tabular}

[a] In an Ar glove box, four NMR tubes were charged with 1 (10-20 mg, 7-15 $\mu \mathrm{mol}), \mathrm{PhSiH}_{3}(308 \mu \mathrm{L}, 2.5 \mathrm{mmol})$ and 1-hexene ( $31 \mu \mathrm{L}, 0.25 \mathrm{mmol})$. Then $\mathrm{C}_{6} \mathrm{D}_{6}$ was added to bring the total volume of the solution to $0.5 \mathrm{~mL}$. The tubes were closed and shaken vigorously, then quickly removed from the glovebox. The reaction mixtures were maintained at $23{ }^{\circ} \mathrm{C}$ and were recorded by ${ }^{1} \mathrm{H}$ NMR spectroscopy at regular intervals. The concentration of 1-hexene during the reaction was determined by the integrals of 1-hexene $(\delta 5.74 \mathrm{ppm})$ standardized to the total integrals of 1-hexene and hydrosilylated product $3 \mathbf{f}(\delta 4.51 \mathrm{ppm})$. 
Table S2. Measurement of the 1-hexene concentration in different $\mathrm{PhSiH}_{3}$ concentration during the hydrosilylation process. ${ }^{[a]}$

\begin{tabular}{|c|c|c|c|c|}
\hline entry & {$\left[\mathrm{PhSiH}_{3}\right](\mathrm{M})$} & time (min) & $\ln \left([\right.$ hexene $\left.] /[\text { hexene }]_{0}\right)$ & $10^{5} \times k_{\mathrm{obs}}\left(\mathrm{s}^{-1}\right)$ \\
\hline 1 & \multirow{5}{*}{3.2520} & 58 & -0.34108 & \multirow{5}{*}{6.600} \\
\hline 2 & & 183 & -0.79186 & \\
\hline 3 & & 285 & -1.22758 & \\
\hline 4 & & 381 & -1.54646 & \\
\hline 5 & & 537 & -2.24432 & \\
\hline 6 & \multirow{5}{*}{1.6260} & 61 & -0.28768 & \multirow{5}{*}{6.050} \\
\hline 7 & & 183 & -0.72981 & \\
\hline 8 & & 288 & -1.10866 & \\
\hline 9 & & 383 & -1.44817 & \\
\hline 10 & & 543 & -2.04022 & \\
\hline 11 & \multirow{5}{*}{0.8130} & 64 & -0.31745 & \multirow{5}{*}{6.633} \\
\hline 12 & & 188 & -0.79186 & \\
\hline 13 & & 291 & -1.20731 & \\
\hline 14 & & 385 & -1.58964 & \\
\hline 15 & & 540 & -2.20727 & \\
\hline
\end{tabular}

[a] In an Ar glove box, three NMR tubes were charged with 1 (8 mg, $6 \mu \mathrm{mol}), \mathrm{PhSiH}_{3}(50-200 \mu \mathrm{L}, 0.4-1.6 \mathrm{mmol})$ and 1-hexene ( $31 \mu \mathrm{L}, 0.25 \mathrm{mmol})$. Then $\mathrm{C}_{6} \mathrm{D}_{6}$ was added to bring the total volume of the solution to $0.5 \mathrm{~mL}$. The tubes were closed and shaken vigorously, then quickly removed from the glovebox. The reaction mixtures were maintained at $23{ }^{\circ} \mathrm{C}$ and were recorded by ${ }^{1} \mathrm{H}$ NMR spectroscopy at regular intervals. The concentration of 1-hexene during the reaction was determined by the integrals of 1-hexene $(\delta 5.74 \mathrm{ppm})$ standardized to the total integrals of 1-hexene and hydrosilylated product $3 \mathbf{f}(\delta 4.51 \mathrm{ppm})$. 


\section{X-ray Diffraction Parameters and Data}

All intensity data were collected with a Bruker SMART CCD diffractometer, using graphite-monochromated Mo K $\alpha$ radiation $(\lambda=0.71073 \AA$ ). The structures were resolved by direct methods and refined by full matrix least squares on $F^{2} .{ }^{S 7}$ Hydrogen atoms were considered in calculated positions. All non-hydrogen atoms were refined anisotropically. The ORTEP-3 program was utilized to draw the molecules. ${ }^{\mathrm{S} 8}$ Crystal data and data collection details are collected in Tables S3. Crystals of $\mathbf{1}$ suitable for X-ray analysis was obtained from DME at $-35{ }^{\circ} \mathrm{C}$. CCDC 902001 (1) contains the supplementary crystallographic data for this paper. These data can be obtained free of charge from The Cambridge Crystallographic Data Centre via www.ccdc.cam.ac.uk/data_request/cif.

Table S3. Crystallographic detail for 1.

\begin{tabular}{ll}
\hline & $\mathbf{1}$ \\
\hline formula & $\mathrm{C}_{66} \mathrm{H}_{106} \mathrm{~N}_{4} \mathrm{O}_{6} \mathrm{Sm}_{2} \bullet\left(\mathrm{C}_{4} \mathrm{H}_{10} \mathrm{O}_{2}\right)$ \\
$\mathrm{FW}$ & 1442.37 \\
$T(\mathrm{~K})$ & $113(2)$ \\
space group & $P 2{ }_{1} / n$ \\
$a(\AA)$ & $14.121(3)$ \\
$b(\AA)$ & $16.241(3)$ \\
$c(\AA)$ & $16.420(4)$ \\
$\alpha\left(^{\circ}\right)$ & 90 \\
$\beta\left(^{\circ}\right)$ & $107.644(3)$ \\
$\gamma\left(^{\circ}\right)$ & 90 \\
$V\left({ }^{\circ}{ }^{3}\right)$ & $3588.6(13)$ \\
$Z$ & 2 \\
$d_{\text {calcd }}\left(\mathrm{g} / \mathrm{cm}{ }^{3}\right)$ & 1.335 \\
$F(000)$ & 1504 \\
$\mathrm{GOF}$ & 1.026 \\
$R_{1}, w R_{2}(I>2 \sigma(I))$ & $0.0227,0.0521$ \\
$R_{1}, w R_{2}($ all data $)$ & $0.0267,0.0533$ \\
$\mathrm{CCDC}$ & 902001 \\
\hline
\end{tabular}




\section{Computational Methods}

Geometry optimizations were performed with the $\mathrm{B}^{2} \mathrm{LYP}^{\mathrm{S} 9}$ functional. The large core ECP51MWB ${ }^{\mathrm{S} 10}$ optimized by the Stuttgart/Cologne group was used for samarium centers, and the 6-31G(d) basis set was employed for other atoms. Frequency calculations were performed at $298.15 \mathrm{~K}$ to verify that the stationary points were the local minima or first-order saddle points, i.e., transition states, and to obtain thermal corrections. Intrinsic Reaction Coordinate (IRC) ${ }^{\mathrm{S} 11}$ calculations were performed to determine the connectivity of the minima and transition states. Single point energy calculations were performed with the M06 functional, ${ }^{\mathrm{S} 12}$ a mixed basis set of ECP51MWB ${ }^{\mathrm{S} 10}$ for samarium and 6-311+G(d,p) for other atoms, and the $\mathrm{SMD}^{\mathrm{S} 13}$ solvation model with toluene as the solvent $(\varepsilon=2.3741)$. All the reported Gibbs free energy values are the sum of the electronic energy from the single point calculations and the thermal corrections obtained by the frequency calculations . All of the calculations were performed with Gaussian 09. ${ }^{\text {S14 }}$ 


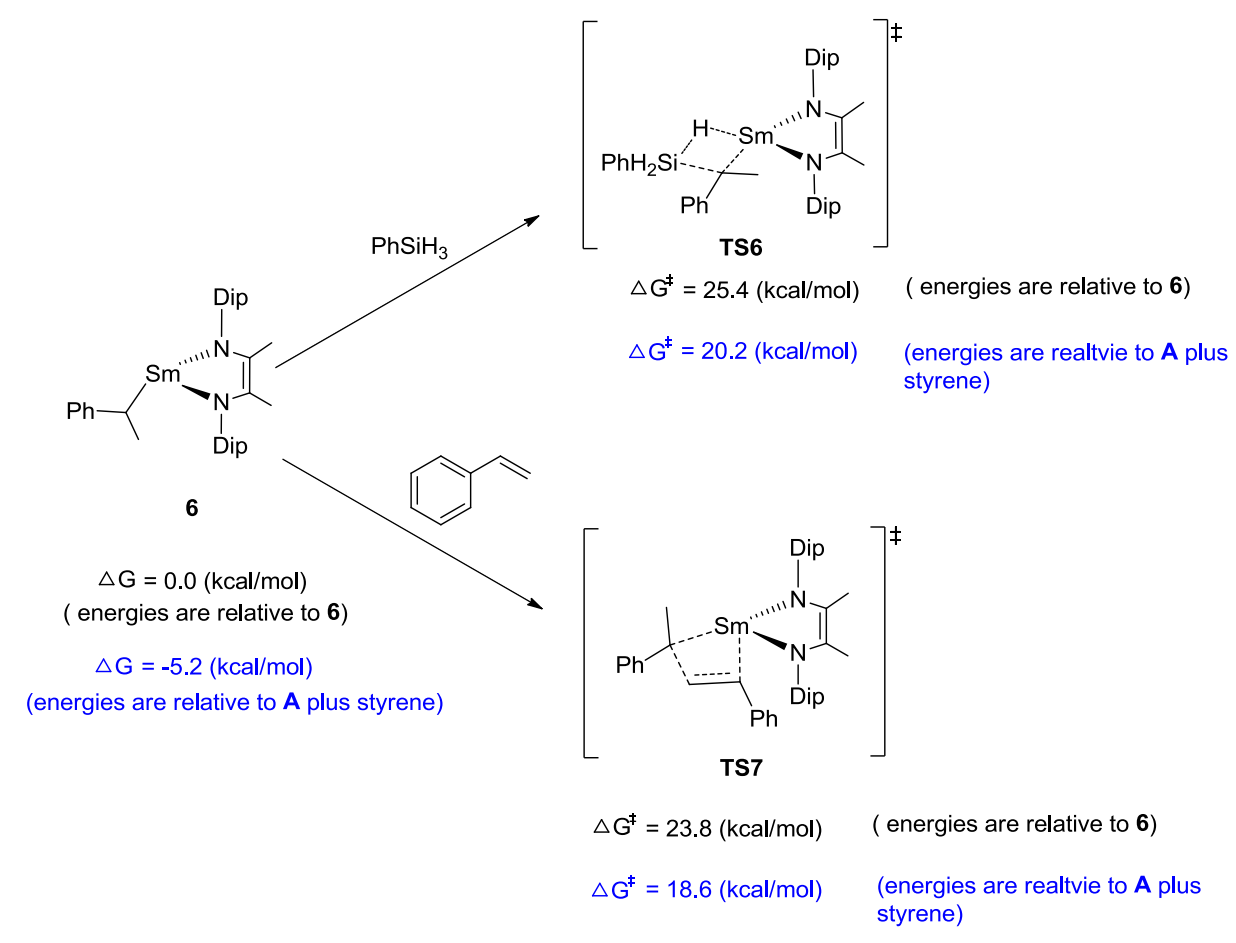

Figure S1. Gibbs free energies of the transition states for the hydrosilylation and polymerization of styrene.

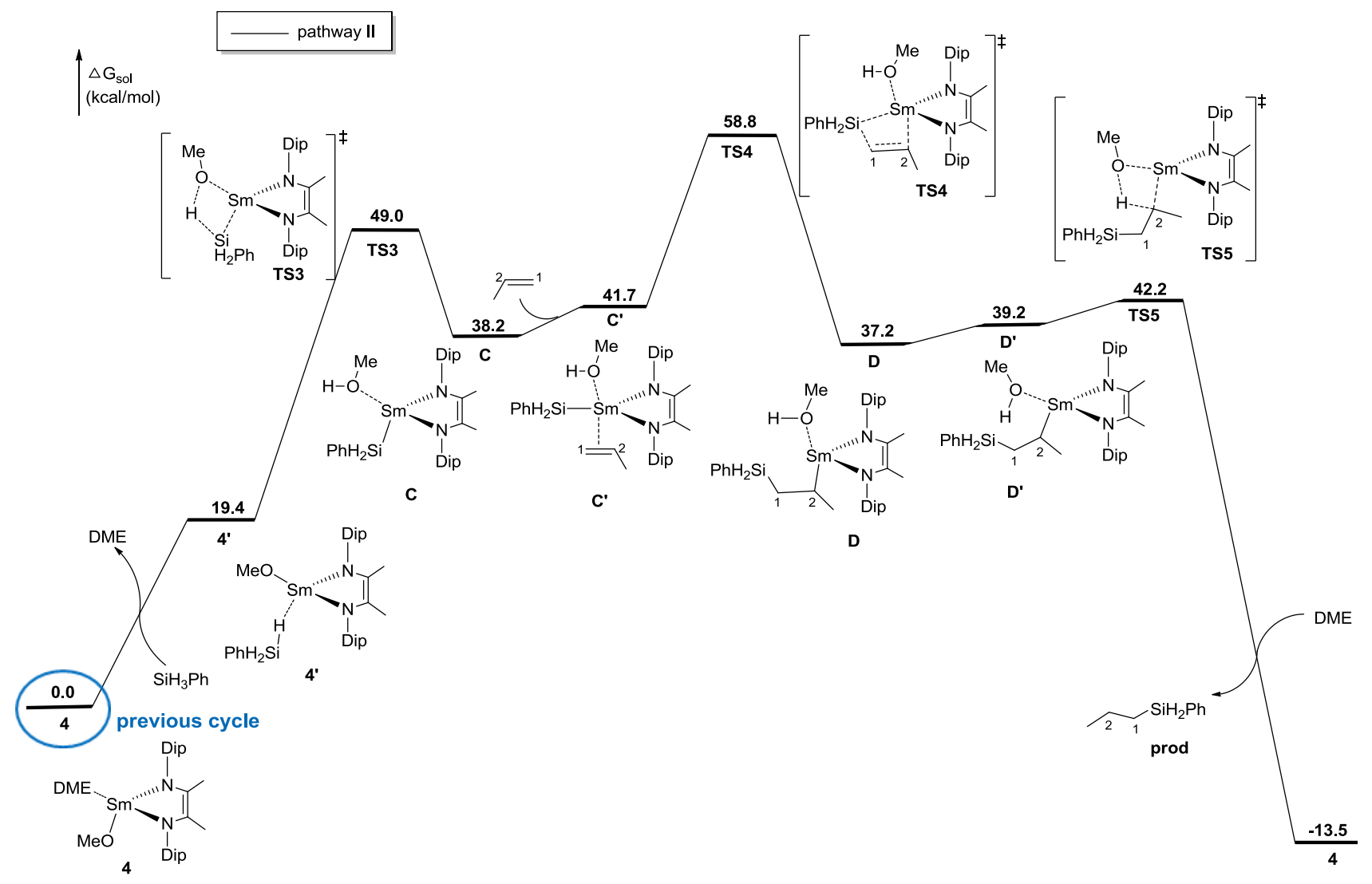

Figure S2. The Gibbs free energy profiles of the 1-catalyzed hydrosilylation of propene along other possible pathways involving samarium silyl intermediate. Energies are calculated using M06/ECP51MWB $-6-311+\mathrm{G}(\mathrm{d}, \mathrm{p}) / \mathrm{SMD}$ (toluene)//B3LYP/ECP51MWB-6-31G(d) method. 
For transition state structures, one imaginary frequency was observed and given below. For all minimum structures, no imaginary frequency was observed.

4

$\begin{array}{llll}\text { Atom } & \mathrm{X} & \mathrm{Y} & \mathrm{Z}\end{array}$

$\begin{array}{llll}\mathrm{O} & 0.530203 & -3.525459 & 1.253153\end{array}$

$\begin{array}{llll}\mathrm{O} & 0.210946 & -2.820561 & -1.345277\end{array}$

$\begin{array}{llll}\mathrm{N} & -1.486868 & 0.411324 & -0.458474\end{array}$

$\mathrm{N} \quad 1.392542 \quad 0.599988-0.389925$

$\begin{array}{llll}\text { C } & -2.856540 & 0.728890 & -0.313550\end{array}$

$\begin{array}{llll}\text { C } & 2.676801 & 1.156482 & -0.153921\end{array}$

$\begin{array}{llll}\text { C } & 0.642283 & 0.963534 & -1.544190\end{array}$

$\begin{array}{llll}\text { C } & 3.849427 & 0.377002 & -0.369228\end{array}$

C $\quad 1.421732 \quad 1.308038-2.799220$

$\begin{array}{llll}\mathrm{H} & 1.796687 & 0.412198 & -3.322862\end{array}$

$\begin{array}{llll}\mathrm{H} & 2.302528 & 1.908635 & -2.550123\end{array}$

$\mathrm{H} \quad 0.826875 \quad 1.876612 \quad-3.517850$

$\begin{array}{llll}\text { C } & 0.319471 & -3.948539 & 2.608687\end{array}$

$\begin{array}{llll}\mathrm{H} & -0.686745 & -4.371457 & 2.724864\end{array}$

$\begin{array}{llll}\mathrm{H} & 0.414563 & -3.050887 & 3.217904\end{array}$

$\begin{array}{llll}\mathrm{H} & 1.069808 & -4.699547 & 2.885883\end{array}$

$\begin{array}{llll}\text { C } & -3.823257 & -0.321761 & -0.299435\end{array}$

$\begin{array}{llll}\text { C } & -0.739284 & 0.857586 & -1.583218\end{array}$

$\begin{array}{llll}\text { C } & -3.307789 & 2.066977 & -0.124418\end{array}$

$\begin{array}{llll}\text { C } & 0.298894 & -4.556082 & 0.299235\end{array}$

$\begin{array}{llll}\mathrm{H} & -0.775833 & -4.785663 & 0.255459\end{array}$

$\begin{array}{llll}\mathrm{H} & 0.838719 & -5.470425 & 0.584674\end{array}$

$\begin{array}{llll}\text { C } & -3.423812 & -1.776723 & -0.525712\end{array}$

$\mathrm{H} \quad-2.413128 \quad-1.764856 \quad-0.951473$

$\begin{array}{llll}\text { C } & 2.818657 & 2.474779 & 0.362476\end{array}$

$\begin{array}{llll}\text { C } & -1.489947 & 1.071964 & -2.895146\end{array}$

$\mathrm{H} \quad-1.383972 \quad 2.095579 \quad-3.275865$

$\mathrm{H} \quad-2.558414 \quad 0.892763 \quad-2.752892$

$\begin{array}{llll}\mathrm{H} & -1.152195 & 0.403683 & -3.700508\end{array}$

$\begin{array}{llll}\text { C } & -3.376836 & -2.563056 & 0.801565\end{array}$

$\mathrm{H} \quad-2.711848 \quad-2.090212 \quad 1.537356$

$\begin{array}{llll}\mathrm{H} & -3.042075 & -3.597991 & 0.641778\end{array}$

$\begin{array}{llll}\mathrm{H} & -4.370615 & -2.604477 & 1.263251\end{array}$

$\begin{array}{llll}\text { C } & 5.106461 & 0.918775 & -0.072851\end{array}$ 


\begin{tabular}{|c|c|c|c|}
\hline $\mathrm{H}$ & 5.997655 & 0.316118 & -0.234643 \\
\hline $\mathrm{C}$ & 0.404412 & -2.423353 & -2.711428 \\
\hline $\mathrm{H}$ & 1.471533 & -2.428570 & -2.963896 \\
\hline $\mathrm{H}$ & 0.014276 & -1.409609 & -2.800013 \\
\hline $\mathrm{H}$ & -0.138861 & -3.104288 & -3.377876 \\
\hline $\mathrm{C}$ & 3.784469 & -1.044914 & -0.917942 \\
\hline $\mathrm{H}$ & 2.735824 & -1.238376 & -1.168826 \\
\hline $\mathrm{C}$ & 0.802497 & -4.085832 & -1.049512 \\
\hline $\mathrm{H}$ & 1.896763 & -3.985876 & -1.045004 \\
\hline $\mathrm{H}$ & 0.520664 & -4.820818 & -1.816469 \\
\hline $\mathrm{C}$ & 4.097627 & 2.978295 & 0.626126 \\
\hline $\mathrm{H}$ & 4.198362 & 3.987636 & 1.018234 \\
\hline $\mathrm{C}$ & -2.340391 & 3.244630 & -0.065185 \\
\hline $\mathrm{H}$ & -1.340316 & 2.866881 & -0.285254 \\
\hline $\mathrm{C}$ & 1.606795 & 3.346078 & 0.670455 \\
\hline $\mathrm{H}$ & 0.717341 & 2.747505 & 0.462413 \\
\hline C & -5.172245 & -0.024546 & -0.071476 \\
\hline $\mathrm{H}$ & -5.900205 & -0.832080 & -0.050138 \\
\hline $\mathrm{C}$ & 5.241497 & 2.214382 & 0.414217 \\
\hline $\mathrm{H}$ & 6.225513 & 2.621979 & 0.631725 \\
\hline $\mathrm{C}$ & -4.670146 & 2.313582 & 0.077009 \\
\hline $\mathrm{H}$ & -5.003333 & 3.337601 & 0.229005 \\
\hline C & 1.557115 & 4.596046 & -0.229235 \\
\hline $\mathrm{H}$ & 2.427764 & 5.243379 & -0.065431 \\
\hline $\mathrm{H}$ & 0.658389 & 5.189161 & -0.018806 \\
\hline $\mathrm{H}$ & 1.539918 & 4.320767 & -1.289485 \\
\hline C & 1.560414 & 3.739271 & 2.160566 \\
\hline $\mathrm{H}$ & 1.564012 & 2.850751 & 2.802255 \\
\hline $\mathrm{H}$ & 0.651133 & 4.311102 & 2.379649 \\
\hline $\mathrm{H}$ & 2.419105 & 4.359798 & 2.444962 \\
\hline $\mathrm{C}$ & -4.320001 & -2.504046 & -1.545926 \\
\hline $\mathrm{H}$ & -5.344013 & -2.634046 & -1.177367 \\
\hline $\mathrm{H}$ & -3.923183 & -3.505034 & -1.759818 \\
\hline $\mathrm{H}$ & -4.373293 & -1.949352 & -2.488886 \\
\hline $\mathrm{C}$ & -2.666373 & 4.327586 & -1.112287 \\
\hline $\mathrm{H}$ & -2.684160 & 3.911392 & -2.125503 \\
\hline $\mathrm{H}$ & -1.912761 & 5.124213 & -1.086249 \\
\hline $\mathrm{H}$ & -3.642947 & 4.791724 & -0.927267 \\
\hline $\mathrm{C}$ & -5.605967 & 1.283908 & 0.112179 \\
\hline $\mathrm{H}$ & -6.657930 & 1.498411 & 0.282073 \\
\hline
\end{tabular}




$\begin{array}{llll}\mathrm{C} & -2.304604 & 3.852409 & 1.351566 \\ \mathrm{H} & -3.284887 & 4.249102 & 1.643107 \\ \mathrm{H} & -1.584075 & 4.678230 & 1.400981 \\ \mathrm{H} & -2.013478 & 3.101587 & 2.094430 \\ \mathrm{C} & 4.603959 & -1.214159 & -2.211854 \\ \mathrm{H} & 4.284647 & -0.498103 & -2.976873 \\ \mathrm{H} & 4.484861 & -2.227186 & -2.619447 \\ \mathrm{H} & 5.674845 & -1.057552 & -2.037786 \\ \mathrm{C} & 4.213670 & -2.081858 & 0.139165 \\ \mathrm{H} & 5.251933 & -1.923112 & 0.453318 \\ \mathrm{H} & 4.142876 & -3.104437 & -0.257279 \\ \mathrm{H} & 3.588528 & -2.018890 & 1.038657 \\ \mathrm{O} & -0.047842 & -0.780555 & 2.721878 \\ \mathrm{C} & -0.146430 & -0.292620 & 4.024797 \\ \mathrm{H} & 0.640495 & 0.447218 & 4.243354 \\ \mathrm{H} & -1.117544 & 0.195827 & 4.204524 \\ \mathrm{H} & -0.046553 & -1.102013 & 4.770482 \\ \mathrm{Sm} & 0.036213 & -0.958515 & 0.580975\end{array}$

TS

Imaginary frequency: $-\mathbf{- 2 5 6 . 1 1 1} \mathrm{cm}^{-1}$

$\begin{array}{lrcc}\text { Atom } & \mathrm{X} & \mathrm{Y} & \mathrm{Z} \\ \mathrm{N} & 1.791057 & -0.951875 & 0.138418 \\ \mathrm{~N} & 0.340496 & 1.559979 & 0.060041 \\ \mathrm{C} & 2.846897 & -1.867527 & -0.028561 \\ \mathrm{C} & 0.105750 & 2.939989 & -0.180006 \\ \mathrm{C} & 1.011944 & 1.116802 & 1.227175 \\ \mathrm{C} & -1.206527 & 3.480367 & -0.075518 \\ \mathrm{C} & 0.830554 & 1.934125 & 2.495976 \\ \mathrm{H} & -0.103721 & 1.692788 & 3.026622 \\ \mathrm{H} & 0.780277 & 2.998031 & 2.247643 \\ \mathrm{H} & 1.652237 & 1.794448 & 3.202377 \\ \mathrm{C} & 2.532932 & -3.188564 & -0.485233 \\ \mathrm{C} & 1.692010 & -0.104066 & 1.273398 \\ \mathrm{C} & 4.218262 & -1.533084 & 0.181893 \\ \mathrm{C} & 1.104539 & -3.547465 & -0.868799 \\ \mathrm{H} & 0.442385 & -3.100616 & -0.106388 \\ \mathrm{C} & 1.172417 & 3.787332 & -0.588690 \\ \mathrm{C} & 2.183984 & -0.641547 & 2.615360\end{array}$




\begin{tabular}{|c|c|c|c|}
\hline $\mathrm{H}$ & 2.584581 & -1.650143 & 2.492306 \\
\hline $\mathrm{H}$ & 1.384691 & -0.689720 & 3.365965 \\
\hline $\mathrm{H}$ & 2.981708 & -0.024848 & 3.046041 \\
\hline $\mathrm{C}$ & 0.752296 & -2.942687 & -2.248977 \\
\hline $\mathrm{H}$ & 1.057975 & -1.886454 & -2.327020 \\
\hline $\mathrm{H}$ & -0.315950 & -3.028501 & -2.480209 \\
\hline $\mathrm{H}$ & 1.316013 & -3.458478 & -3.034965 \\
\hline $\mathrm{C}$ & -1.921432 & -1.275895 & 2.633146 \\
\hline $\mathrm{H}$ & -1.052143 & -0.645866 & 2.837855 \\
\hline $\mathrm{H}$ & -1.816297 & -2.224316 & 3.167463 \\
\hline $\mathrm{H}$ & -2.826302 & -0.761865 & 2.971870 \\
\hline $\mathrm{C}$ & -1.417450 & 4.831570 & -0.379461 \\
\hline $\mathrm{H}$ & -2.422141 & 5.240601 & -0.305279 \\
\hline C & -2.409299 & 2.645469 & 0.351768 \\
\hline $\mathrm{H}$ & -2.035375 & 1.655686 & 0.644298 \\
\hline & 0.911494 & 5.133915 & -0.864412 \\
\hline & 1.729822 & 5.778533 & -1.175739 \\
\hline $\mathrm{C}$ & 4.697950 & -0.103547 & 0.433352 \\
\hline $\mathrm{H}$ & 3.831569 & 0.521673 & 0.658425 \\
\hline $\mathrm{C}$ & 2.592761 & 3.261751 & -0.767412 \\
\hline $\mathrm{H}$ & 2.579065 & 2.191151 & -0.549704 \\
\hline $\mathrm{C}$ & 3.548640 & -4.136108 & -0.628318 \\
\hline $\mathrm{H}$ & 3.300830 & -5.139693 & -0.958824 \\
\hline C & -0.372053 & 5.662775 & -0.765900 \\
\hline $\mathrm{H}$ & -0.555976 & 6.709684 & -0.992879 \\
\hline C & 5.193736 & -2.527117 & 0.032444 \\
\hline $\mathrm{H}$ & 6.235873 & -2.267436 & 0.199690 \\
\hline $\mathrm{C}$ & 3.575822 & 3.932100 & 0.212581 \\
\hline $\mathrm{H}$ & 3.648262 & 5.011826 & 0.033124 \\
\hline $\mathrm{H}$ & 4.582100 & 3.510458 & 0.101379 \\
\hline $\mathrm{H}$ & 3.261788 & 3.789289 & 1.252652 \\
\hline $\mathrm{C}$ & 3.073018 & 3.425546 & -2.223454 \\
\hline $\mathrm{H}$ & 2.389002 & 2.929630 & -2.921826 \\
\hline $\mathrm{H}$ & 4.068614 & 2.985039 & -2.352739 \\
\hline $\mathrm{H}$ & 3.135939 & 4.481188 & -2.514016 \\
\hline $\mathrm{C}$ & 0.775726 & -5.048869 & -0.867589 \\
\hline $\mathrm{H}$ & 1.301874 & -5.583547 & -1.666585 \\
\hline $\mathrm{H}$ & -0.298103 & -5.194878 & -1.031543 \\
\hline $\mathrm{H}$ & 1.042667 & -5.515508 & 0.086551 \\
\hline $\mathrm{C}$ & 5.674641 & 0.016929 & 1.619604 \\
\hline
\end{tabular}




$\begin{array}{llll}\mathrm{H} & 5.255158 & -0.414383 & 2.534738 \\ \mathrm{H} & 5.906278 & 1.071006 & 1.815071 \\ \mathrm{H} & 6.625218 & -0.491799 & 1.420191 \\ \mathrm{C} & 4.877122 & -3.824326 & -0.352130 \\ \mathrm{H} & 5.654518 & -4.575829 & -0.459761 \\ \mathrm{C} & 5.342764 & 0.461606 & -0.849979 \\ \mathrm{H} & 6.225366 & -0.121820 & -1.138600 \\ \mathrm{H} & 5.661480 & 1.500699 & -0.700688 \\ \mathrm{H} & 4.637890 & 0.437600 & -1.688019 \\ \mathrm{C} & -3.121222 & 3.226770 & 1.588415 \\ \mathrm{H} & -2.426172 & 3.344423 & 2.427398 \\ \mathrm{H} & -3.938027 & 2.567155 & 1.907085 \\ \mathrm{H} & -3.556841 & 4.210447 & 1.378986 \\ \mathrm{C} & -3.402814 & 2.442652 & -0.809007 \\ \mathrm{H} & -3.822118 & 3.401128 & -1.137749 \\ \mathrm{H} & -4.233685 & 1.794008 & -0.509149 \\ \mathrm{H} & -2.914934 & 1.983965 & -1.677578 \\ \mathrm{Sm} & -0.392943 & -0.532458 & -0.391917 \\ \mathrm{C} & -4.813800 & -1.690351 & -0.005941 \\ \mathrm{C} & -5.898786 & -1.692859 & 0.894645 \\ \mathrm{C} & -5.016589 & -1.132110 & -1.282887 \\ \mathrm{C} & -7.135081 & -1.149423 & 0.540303 \\ \mathrm{H} & -5.781568 & -2.131798 & 1.882901 \\ \mathrm{C} & -6.254756 & -0.599172 & -1.642007 \\ \mathrm{H} & -4.186836 & -1.112035 & -1.982797 \\ \mathrm{C} & -7.314427 & -0.602267 & -0.730779 \\ \mathrm{H} & -7.955700 & -1.158118 & 1.252977 \\ \mathrm{H} & -6.392522 & -0.177323 & -2.634240 \\ \mathrm{H} & -8.276324 & -0.180780 & -1.011640 \\ \mathrm{O} & -1.974411 & -1.501535 & 1.208346 \\ \mathrm{H} & -2.125114 & -1.360995 & -1.469664 \\ \mathrm{H} & -3.235793 & -2.522943 & 0.561720 \\ & -3.640825 & -3.210822 & 1.828848 \\ & -2.644662 & -3.560060 & -0.306040\end{array}$

5

$\begin{array}{lrccc}\text { Atom } & \mathrm{X} & \mathrm{Y} & \mathrm{Z} \\ \mathrm{N} & -0.497422 & 1.572769 & 0.229835 \\ \mathrm{~N} & -1.704914 & -1.034479 & 0.115024 \\ \mathrm{C} & -0.348700 & 2.978241 & 0.134367\end{array}$




\begin{tabular}{|c|c|c|c|}
\hline$C$ & -2.672728 & -2.055414 & -0.095409 \\
\hline $\mathrm{C}$ & -1.672832 & -0.284847 & 1.321512 \\
\hline $\mathrm{C}$ & -2.325417 & -3.424967 & 0.076400 \\
\hline $\mathrm{C}$ & -2.182775 & -0.956752 & 2.582757 \\
\hline & -1.458303 & -1.671812 & 3.003857 \\
\hline $\mathrm{H}$ & -3.090846 & -1.527959 & 2.365575 \\
\hline $\mathrm{H}$ & -2.425855 & -0.235507 & 3.366110 \\
\hline $\mathrm{C}$ & 0.957702 & 3.541921 & 0.030140 \\
\hline $\mathrm{C}$ & -1.072785 & 0.966694 & 1.382434 \\
\hline $\mathrm{C}$ & -1.474578 & 3.848519 & 0.089875 \\
\hline $\mathrm{C}$ & 2.217875 & 2.684623 & 0.095924 \\
\hline $\mathrm{H}$ & 1.916861 & 1.707515 & 0.498981 \\
\hline $\mathrm{C}$ & -3.981343 & -1.725887 & -0.540844 \\
\hline $\mathrm{C}$ & -0.883619 & 1.669427 & 2.723357 \\
\hline $\mathrm{H}$ & -1.830517 & 2.040754 & 3.136627 \\
\hline $\mathrm{H}$ & -0.227001 & 2.535293 & 2.606350 \\
\hline $\mathrm{H}$ & -0.440803 & 1.018220 & 3.487645 \\
\hline $\mathrm{C}$ & 2.827382 & 2.457699 & -1.302988 \\
\hline $\mathrm{H}$ & 2.114064 & 1.991575 & -1.995972 \\
\hline $\mathrm{H}$ & 3.719429 & 1.820188 & -1.248097 \\
\hline $\mathrm{H}$ & 3.125605 & 3.412735 & -1.751753 \\
\hline $\mathrm{C}$ & 2.258629 & -1.005327 & 1.994605 \\
\hline $\mathrm{H}$ & 1.341848 & -0.484172 & 2.280405 \\
\hline $\mathrm{H}$ & 3.120799 & -0.353820 & 2.167113 \\
\hline $\mathrm{H}$ & 2.352874 & -1.919292 & 2.590642 \\
\hline $\mathrm{C}$ & -3.278017 & -4.415222 & -0.194324 \\
\hline $\mathrm{H}$ & -3.008995 & -5.461470 & -0.069198 \\
\hline $\mathrm{C}$ & -0.938092 & -3.861032 & 0.537871 \\
\hline $\mathrm{H}$ & -0.390893 & -2.953998 & 0.819276 \\
\hline $\mathrm{C}$ & -4.902830 & -2.751385 & -0.782609 \\
\hline $\mathrm{H}$ & -5.904053 & -2.495207 & -1.120527 \\
\hline $\mathrm{C}$ & -2.907875 & 3.326867 & 0.131158 \\
\hline $\mathrm{H}$ & -2.869936 & 2.249733 & 0.304615 \\
\hline $\mathrm{C}$ & -4.402037 & -0.282049 & -0.785206 \\
\hline $\mathrm{H}$ & -3.520976 & 0.343100 & -0.620906 \\
\hline $\mathrm{C}$ & 1.102937 & 4.924065 & -0.141097 \\
\hline $\mathrm{H}$ & 2.099730 & 5.347775 & -0.234810 \\
\hline $\mathrm{C}$ & -4.563438 & -4.090152 & -0.614161 \\
\hline $\mathrm{H}$ & -5.291183 & -4.872436 & -0.814047 \\
\hline $\mathrm{C}$ & -1.273258 & 5.224774 & -0.060808 \\
\hline
\end{tabular}




\begin{tabular}{|c|c|c|c|}
\hline $\mathrm{H}$ & -2.137837 & 5.882822 & -0.103030 \\
\hline $\mathrm{C}$ & -5.492281 & 0.166722 & 0.207280 \\
\hline $\mathrm{H}$ & -6.407619 & -0.427296 & 0.093360 \\
\hline $\mathrm{H}$ & -5.757114 & 1.218550 & 0.043414 \\
\hline $\mathrm{H}$ & -5.152906 & 0.061017 & 1.243740 \\
\hline $\mathrm{C}$ & -4.857260 & -0.062887 & -2.241489 \\
\hline $\mathrm{H}$ & -4.073944 & -0.359049 & -2.948485 \\
\hline $\mathrm{H}$ & -5.089421 & 0.994101 & -2.416422 \\
\hline $\mathrm{H}$ & -5.756936 & -0.643453 & -2.479429 \\
\hline $\mathrm{C}$ & 3.280382 & 3.240770 & 1.063179 \\
\hline $\mathrm{H}$ & 3.704540 & 4.186127 & 0.706614 \\
\hline $\mathrm{H}$ & 4.112138 & 2.532151 & 1.165091 \\
\hline $\mathrm{H}$ & 2.856608 & 3.420390 & 2.057405 \\
\hline $\mathrm{C}$ & -3.727811 & 3.950215 & 1.277940 \\
\hline $\mathrm{H}$ & -3.244481 & 3.791200 & 2.248248 \\
\hline $\mathrm{H}$ & -4.728218 & 3.502611 & 1.320233 \\
\hline $\mathrm{H}$ & -3.856292 & 5.031360 & 1.145479 \\
\hline $\mathrm{C}$ & 0.000824 & 5.770508 & -0.183312 \\
\hline $\mathrm{H}$ & 0.132794 & 6.841501 & -0.313121 \\
\hline $\mathrm{C}$ & -3.608557 & 3.555298 & -1.223464 \\
\hline $\mathrm{H}$ & -3.679663 & 4.623565 & -1.461472 \\
\hline $\mathrm{H}$ & -4.627495 & 3.149833 & -1.205980 \\
\hline $\mathrm{H}$ & -3.061669 & 3.067729 & -2.037980 \\
\hline C & -0.983126 & -4.763018 & 1.786238 \\
\hline $\mathrm{H}$ & -1.518884 & -4.275510 & 2.607922 \\
\hline $\mathrm{H}$ & 0.032871 & -4.996263 & 2.130064 \\
\hline $\mathrm{H}$ & -1.484804 & -5.715588 & 1.581275 \\
\hline $\mathrm{C}$ & -0.155649 & -4.547180 & -0.600358 \\
\hline $\mathrm{H}$ & -0.659228 & -5.464254 & -0.927765 \\
\hline $\mathrm{H}$ & 0.855677 & -4.824021 & -0.273452 \\
\hline $\mathrm{H}$ & -0.068241 & -3.897264 & -1.480321 \\
\hline $\mathrm{Sm}$ & 0.253536 & -0.323468 & -0.748209 \\
\hline $\mathrm{C}$ & 5.009934 & -1.539795 & -0.249192 \\
\hline $\mathrm{C}$ & 5.338733 & -0.793355 & -1.398614 \\
\hline $\mathrm{C}$ & 5.968747 & -1.642560 & 0.777465 \\
\hline $\mathrm{C}$ & 6.578117 & -0.162318 & -1.511067 \\
\hline $\mathrm{H}$ & 4.626008 & -0.710743 & -2.216412 \\
\hline $\mathrm{C}$ & 7.207566 & -1.010601 & 0.667155 \\
\hline $\mathrm{H}$ & 5.752618 & -2.228527 & 1.668649 \\
\hline $\mathrm{C}$ & 7.511178 & -0.268825 & -0.476877 \\
\hline
\end{tabular}




$\begin{array}{llll}\mathrm{H} & 6.816923 & 0.406587 & -2.405189 \\ \mathrm{H} & 7.936414 & -1.100482 & 1.467941 \\ \mathrm{H} & 8.477210 & 0.220875 & -0.565139 \\ \mathrm{O} & 2.159613 & -1.339112 & 0.589787 \\ \mathrm{Si} & 3.350114 & -2.364863 & -0.107840 \\ \mathrm{H} & 2.765770 & -2.652045 & -1.434712 \\ \mathrm{H} & 3.428294 & -3.557976 & 0.773020 \\ \mathrm{H} & 0.663664 & -0.437791 & -2.856046\end{array}$

\section{A (catalyst)}

Atom $\mathrm{X}$

O $\quad-0.523966$

$\mathrm{Y}$

Z

$\begin{array}{llll}\text { O } & -0.236493 & -2.889081 & 1.102016\end{array}$

$\begin{array}{llll}\mathrm{N} & 1.482889 & 0.401873 & 0.281930\end{array}$

$\begin{array}{llll}\mathrm{N} & -1.374233 & 0.594488 & 0.254297\end{array}$

$\begin{array}{llll}\text { C } & 2.862818 & 0.698000 & 0.151285\end{array}$

$\begin{array}{llll}\text { C } & -2.659485 & 1.153174 & 0.017183\end{array}$

$\begin{array}{llll}\text { C } & -0.625615 & 0.932869 & 1.418848\end{array}$

$\begin{array}{llll}\text { C } & -3.833574 & 0.371752 & 0.214760\end{array}$

$\begin{array}{llll}\text { C } & -1.431184 & 1.272231 & 2.661265\end{array}$

$\begin{array}{llll}\mathrm{H} & -1.818200 & 0.376681 & 3.174219\end{array}$

$\begin{array}{llll}\mathrm{H} & -2.306150 & 1.868396 & 2.384807\end{array}$

$\begin{array}{llll}\mathrm{H} & -0.865949 & 1.852311 & 3.393137\end{array}$

$\begin{array}{llll}\text { C } & -0.342113 & -3.883183 & -2.900318\end{array}$

$\begin{array}{llll}\mathrm{H} & 0.663979 & -4.295410 & -3.049158\end{array}$

$\begin{array}{llll}\mathrm{H} & -0.456279 & -2.973667 & -3.489166\end{array}$

H $\quad-1.094618 \quad-4.633453 \quad-3.174062$

$\begin{array}{llll}\text { C } & 3.814390 & -0.363689 & 0.120097\end{array}$

$\begin{array}{llll}\text { C } & 0.756736 & 0.811514 & 1.438941\end{array}$

$\begin{array}{llll}\text { C } & 3.328420 & 2.034075 & -0.008772\end{array}$

$\begin{array}{llll}\text { C } & -0.288637 & -4.559336 & -0.612296\end{array}$

H $\quad 0.787675 \quad-4.781163 \quad-0.569592$

$\begin{array}{llll}\mathrm{H} & -0.817053 & -5.466719 & -0.937990\end{array}$

$\begin{array}{llll}\text { C } & 3.395787 & -1.821684 & 0.283133\end{array}$

$\mathrm{H} \quad 2.383011 \quad-1.817264 \quad 0.706191$

$\begin{array}{llll}\text { C } & -2.794407 & 2.478605 & -0.481142\end{array}$

$\begin{array}{llll}\text { C } & 1.566987 & 0.931333 & 2.717187\end{array}$

$\begin{array}{llll}\mathrm{H} & 2.369019 & 1.670171 & 2.611339\end{array}$

$\begin{array}{llll}\mathrm{H} & 2.066539 & -0.020713 & 2.953593\end{array}$

$\begin{array}{llll}\mathrm{H} & 0.969286 & 1.212283 & 3.584678\end{array}$ 


\begin{tabular}{|c|c|c|c|}
\hline & 3.346599 & -2.546917 & -1.0 \\
\hline & 2.687374 & -2.038263 & -1.7957 \\
\hline & 3.005549 & -3.586064 & \\
\hline & 4.341483 & -2.572698 & -1.5 \\
\hline & -5.088194 & 0.923442 & -0.0734 \\
\hline & -5.981405 & 0.320756 & 0.07577 \\
\hline & -0.440127 & -2.546456 & .4823 \\
\hline & -1.507409 & -2.582347 & 2.7309 \\
\hline & -0.069858 & -1.528550 & 2.6105 \\
\hline & 0.115881 & -3.240671 & 3.12350 \\
\hline & -3.779057 & -1.061485 & 0.7349 \\
\hline & -2.730899 & -1.274215 & 0.9726 \\
\hline & -0.807839 & -4.152264 & 0.7507 \\
\hline & -1.902938 & -4.065957 & \\
\hline & -0.519504 & -4.911437 & 1.4907 \\
\hline & -4.071600 & 2.991013 & -0.7362 \\
\hline & -4.168202 & 4.006623 & -1.1122 \\
\hline & 2.370433 & 3.219764 & -0.07397 \\
\hline & 1.370266 & 2.856308 & 0.169116 \\
\hline & -1.579784 & 3.350204 & -0.7775 \\
\hline & -0.691389 & 2.744454 & -0.5860 \\
\hline & 5.170008 & -0.076296 & -0.0796 \\
\hline & 5.889135 & -0.891101 & -0.1125 \\
\hline & -5.218087 & 2.227999 & -0.5368 \\
\hline & -6.200480 & 2.642767 & -0.7477 \\
\hline & 4.696236 & 2.270343 & -0.1835 \\
\hline 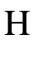 & 5.042490 & 3.293158 & -0.31122 \\
\hline 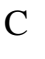 & -1.520623 & 4.582091 & 0.1463 \\
\hline ה & -2.389937 & 5.235469 & 0.0003 \\
\hline & -0.621210 & 5.175841 & -0.0587 \\
\hline 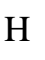 & -1.497341 & 4.287229 & 1.2013 \\
\hline & -1.539230 & 3.770461 & -2.2603 \\
\hline & -1.546432 & 2.894046 & -2.9178 \\
\hline & -0.629611 & 4.344318 & -2.4718 \\
\hline & -2.397345 & 4.398699 & -2.5290 \\
\hline & 4.274704 & -2.604964 & 1.27633 \\
\hline & 5.298169 & -2.735406 & 0.906937 \\
\hline & 3.861525 & -3.607891 & 1.44519 \\
\hline & 4.331813 & -2.091438 & 2.242140 \\
\hline & 2.716965 & 4.326516 & 0.940893 \\
\hline
\end{tabular}




$\begin{array}{llll}\mathrm{H} & 2.742114 & 3.939681 & 1.965854 \\ \mathrm{H} & 1.967112 & 5.125988 & 0.901750 \\ \mathrm{H} & 3.693009 & 4.781867 & 0.733657 \\ \mathrm{C} & 5.620936 & 1.231090 & -0.223847 \\ \mathrm{H} & 6.677846 & 1.437155 & -0.371695 \\ \mathrm{C} & 2.321727 & 3.791913 & -1.505315 \\ \mathrm{H} & 3.304987 & 4.163451 & -1.819253 \\ \mathrm{H} & 1.614698 & 4.628544 & -1.565500 \\ \mathrm{H} & 2.007465 & 3.027386 & -2.224156 \\ \mathrm{C} & -4.586941 & -1.246077 & 2.034219 \\ \mathrm{H} & -4.251086 & -0.549668 & 2.810158 \\ \mathrm{H} & -4.477169 & -2.268629 & 2.420002 \\ \mathrm{H} & -5.657167 & -1.072051 & 1.873739 \\ \mathrm{C} & -4.231947 & -2.072977 & -0.337040 \\ \mathrm{H} & -5.273604 & -1.900235 & -0.631575 \\ \mathrm{H} & -4.163291 & -3.103748 & 0.037840 \\ \mathrm{H} & -3.621003 & -1.996382 & -1.245020 \\ \mathrm{Sm} & -0.042700 & -0.967121 & -0.704224 \\ \mathrm{H} & 0.009772 & -0.823572 & -2.845637\end{array}$

\section{DME}

\begin{tabular}{llll} 
Atom & \multicolumn{1}{c}{$\mathrm{X}$} & $\mathrm{Y}$ & $\mathrm{Z}$ \\
$\mathrm{O}$ & -1.408873 & -0.335660 & -0.372374 \\
$\mathrm{O}$ & 1.409176 & -0.334846 & 0.373203 \\
$\mathrm{C}$ & -2.744605 & -0.441651 & 0.063444 \\
$\mathrm{H}$ & -2.808175 & -0.617714 & 1.149780 \\
$\mathrm{H}$ & -3.188910 & -1.292803 & -0.459397 \\
$\mathrm{H}$ & -3.327273 & 0.465033 & -0.172380 \\
$\mathrm{C}$ & -0.721328 & 0.737753 & 0.233553 \\
$\mathrm{H}$ & -0.747095 & 0.651496 & 1.332716 \\
$\mathrm{H}$ & -1.188677 & 1.701320 & -0.038201 \\
$\mathrm{C}$ & 2.744458 & -0.441899 & -0.063762 \\
$\mathrm{H}$ & 2.806844 & -0.620798 & -1.149698 \\
$\mathrm{H}$ & 3.189399 & -1.291622 & 0.460864 \\
$\mathrm{H}$ & 3.327326 & 0.465433 & 0.169057 \\
$\mathrm{C}$ & 0.721273 & 0.737734 & -0.233810 \\
$\mathrm{H}$ & 0.747007 & 0.650366 & -1.332881 \\
$\mathrm{H}$ & 1.188349 & 1.701710 & 0.036959
\end{tabular}

\section{$\mathrm{PhSiH}_{3}$}




\begin{tabular}{llll} 
Atom & \multicolumn{1}{c}{$\mathrm{X}$} & \multicolumn{1}{c}{$\mathrm{Y}$} & $\mathrm{Z}$ \\
$\mathrm{Si}$ & -2.347967 & -0.000054 & 0.005708 \\
$\mathrm{H}$ & -2.863978 & -1.209295 & -0.692375 \\
$\mathrm{H}$ & -2.895431 & -0.008549 & 1.392025 \\
$\mathrm{H}$ & -2.862302 & 1.218802 & -0.676702 \\
$\mathrm{C}$ & -0.468730 & -0.000300 & -0.011519 \\
$\mathrm{C}$ & 0.256828 & -1.205465 & -0.009349 \\
$\mathrm{C}$ & 0.256371 & 1.205189 & -0.009381 \\
$\mathrm{C}$ & 1.652632 & -1.207802 & 0.003236 \\
$\mathrm{H}$ & -0.271575 & -2.156536 & -0.022704 \\
$\mathrm{C}$ & 1.652177 & 1.208048 & 0.003215 \\
$\mathrm{H}$ & -0.272516 & 2.155959 & -0.022742 \\
$\mathrm{C}$ & 2.352778 & 0.000248 & 0.010349 \\
$\mathrm{H}$ & 2.193038 & -2.150951 & 0.003172 \\
$\mathrm{H}$ & 2.192273 & 2.151369 & 0.003068 \\
$\mathrm{H}$ & 3.439683 & 0.000441 & 0.017044
\end{tabular}

\section{$\mathrm{MeOSiH}_{2} \mathrm{Ph}$}

$\begin{array}{lccc}\text { Atom } & \mathrm{X} & \mathrm{Y} & \mathrm{Z} \\ \mathrm{C} & 2.737459 & 0.498798 & 1.369955 \\ \mathrm{H} & 3.520853 & 0.102793 & 2.023242 \\ \mathrm{H} & 1.814503 & 0.606293 & 1.955894 \\ \mathrm{H} & 3.047357 & 1.493858 & 1.019285 \\ \mathrm{C} & -0.291112 & -0.062089 & -0.405987 \\ \mathrm{C} & -0.975344 & -1.217915 & 0.018024 \\ \mathrm{C} & -0.993291 & 1.156977 & -0.399702 \\ \mathrm{C} & -2.304821 & -1.157402 & 0.435698 \\ \mathrm{H} & -0.464459 & -2.178844 & 0.019648 \\ \mathrm{C} & -2.324813 & 1.223774 & 0.016074 \\ \mathrm{H} & -0.495684 & 2.067143 & -0.728727 \\ \mathrm{C} & -2.981331 & 0.065446 & 0.435221 \\ \mathrm{H} & -2.815218 & -2.061440 & 0.757911 \\ \mathrm{H} & -2.848947 & 2.176079 & 0.011387 \\ \mathrm{H} & -4.018125 & 0.113578 & 0.758290 \\ \mathrm{O} & 2.568449 & -0.406196 & 0.289769 \\ \mathrm{Si} & 1.499328 & -0.141212 & -0.959392 \\ \mathrm{H} & 1.716547 & -1.288747 & -1.873378 \\ \mathrm{H} & 1.804500 & 1.150296 & -1.645921\end{array}$

propene 


$\begin{array}{lccc}\text { Atom } & \mathrm{X} & \mathrm{Y} & \mathrm{Z} \\ \mathrm{C} & -1.283261 & 0.220517 & 0.000005 \\ \mathrm{H} & -1.305306 & 1.308787 & -0.000020 \\ \mathrm{H} & -2.245547 & -0.284554 & 0.000012 \\ \mathrm{C} & -0.133802 & -0.455815 & 0.000002 \\ \mathrm{H} & -0.164038 & -1.546546 & -0.000042 \\ \mathrm{C} & 1.235156 & 0.162966 & 0.000000 \\ \mathrm{H} & 1.181287 & 1.256917 & -0.001170 \\ \mathrm{H} & 1.812109 & -0.149390 & 0.881107 \\ \mathrm{H} & 1.812932 & -0.151222 & -0.879926\end{array}$

\section{styrene}

\begin{tabular}{lllc} 
Atom & \multicolumn{1}{c}{$\mathrm{X}$} & $\mathrm{Y}$ & $\mathrm{Z}$ \\
$\mathrm{C}$ & -1.781065 & -1.046647 & 0.000021 \\
$\mathrm{C}$ & -0.406220 & -1.281583 & 0.000010 \\
$\mathrm{C}$ & 0.515517 & -0.220215 & -0.000037 \\
$\mathrm{C}$ & 0.008686 & 1.092889 & -0.000024 \\
$\mathrm{C}$ & -1.362746 & 1.329834 & 0.000002 \\
$\mathrm{C}$ & -2.265840 & 0.261600 & 0.000006 \\
$\mathrm{H}$ & -2.472108 & -1.885463 & 0.000034 \\
$\mathrm{H}$ & -0.034613 & -2.303949 & 0.000014 \\
$\mathrm{H}$ & 0.693398 & 1.936064 & -0.000047 \\
$\mathrm{H}$ & -1.731202 & 2.352417 & -0.000003 \\
$\mathrm{H}$ & -3.335973 & 0.450057 & 0.000020 \\
$\mathrm{C}$ & 1.955119 & -0.529311 & -0.000039 \\
$\mathrm{H}$ & 2.186202 & -1.594921 & -0.000064 \\
$\mathrm{C}$ & 2.978035 & 0.335085 & 0.000040 \\
$\mathrm{H}$ & 2.840344 & 1.413073 & 0.000114 \\
$\mathrm{H}$ & 4.005040 & -0.017194 & 0.000052
\end{tabular}

\section{prod (product)}

\begin{tabular}{lccc} 
Atom & $\mathrm{X}$ & \multicolumn{1}{c}{$\mathrm{Y}$} & $\mathrm{Z}$ \\
$\mathrm{C}$ & -1.015016 & -0.070096 & 0.381134 \\
$\mathrm{C}$ & -1.665487 & 1.174163 & 0.284042 \\
$\mathrm{C}$ & -1.721577 & -1.212434 & -0.038064 \\
$\mathrm{C}$ & -2.963811 & 1.276093 & -0.218126 \\
$\mathrm{H}$ & -1.155498 & 2.078448 & 0.610962 \\
$\mathrm{C}$ & -3.020391 & -1.117224 & -0.541253 \\
$\mathrm{H}$ & -1.255333 & -2.193104 & 0.034248 \\
$\mathrm{C}$ & -3.643602 & 0.128843 & -0.632917
\end{tabular}




$\begin{array}{llll}\mathrm{H} & -3.446576 & 2.248100 & -0.281269 \\ \mathrm{H} & -3.547123 & -2.014318 & -0.856892 \\ \mathrm{H} & -4.655847 & 0.205238 & -1.021632 \\ \mathrm{C} & 4.518877 & 0.184611 & -1.004438 \\ \mathrm{H} & 4.395972 & 1.174022 & -1.462071 \\ \mathrm{H} & 5.548581 & 0.112433 & -0.634406 \\ \mathrm{H} & 4.394412 & -0.563936 & -1.796676 \\ \mathrm{C} & 3.501911 & -0.031450 & 0.123109 \\ \mathrm{H} & 3.675050 & -1.014369 & 0.582557 \\ \mathrm{C} & 2.040461 & 0.064130 & -0.355976 \\ \mathrm{H} & 1.859482 & -0.674455 & -1.150548 \\ \mathrm{H} & 1.863332 & 1.046725 & -0.817417 \\ \mathrm{Si} & 0.753534 & -0.197880 & 1.013962 \\ \mathrm{H} & 0.945902 & -1.548967 & 1.617364 \\ \mathrm{H} & 0.961390 & 0.826221 & 2.079376 \\ \mathrm{H} & 3.678588 & 0.708466 & 0.915876\end{array}$

$\mathbf{A}^{\prime}$

$\begin{array}{llll}\text { Atom } & \mathrm{X} & \mathrm{Y} & \mathrm{Z}\end{array}$

$\begin{array}{llll}\text { Sm } & 0.052182 & -1.476162 & 0.005733\end{array}$

$\begin{array}{llll}\mathrm{N} & 1.368424 & 0.312410 & 0.328927\end{array}$

$\begin{array}{llll}\mathrm{N} & -1.525101 & 0.097726 & 0.356655\end{array}$

$\begin{array}{llll}\text { C } & 2.647239 & 0.857022 & 0.034496\end{array}$

$\begin{array}{llll}\text { C } & -2.894658 & 0.380342 & 0.157511\end{array}$

$\begin{array}{llll}\text { C } & -0.798655 & 0.656912 & 1.442237\end{array}$

$\begin{array}{llll}\text { C } & -3.834933 & -0.695377 & 0.152892\end{array}$

$\begin{array}{llll}\text { C } & -1.546411 & 0.924323 & 2.741991\end{array}$

$\begin{array}{llll}\mathrm{H} & -1.171993 & 0.300790 & 3.563903\end{array}$

$\begin{array}{llll}\mathrm{H} & -2.610576 & 0.709630 & 2.620682\end{array}$

$\begin{array}{llll}\mathrm{H} & -1.457141 & 1.969017 & 3.064614\end{array}$

$\begin{array}{llll}\text { C } & 3.835884 & 0.169174 & 0.410839\end{array}$

$\begin{array}{llll}\text { C } & 0.590318 & 0.776918 & 1.420810\end{array}$

$\begin{array}{llll}\text { C } & 2.750430 & 2.072804 & -0.695851\end{array}$

$\begin{array}{llll}\text { C } & 3.814861 & -1.125065 & 1.218983\end{array}$

$\begin{array}{llll}\mathrm{H} & 2.772584 & -1.343339 & 1.473933\end{array}$

$\begin{array}{llll}\text { C } & -3.365285 & 1.702327 & -0.081297\end{array}$

$\begin{array}{llll}\text { C } & 1.317500 & 1.225131 & 2.676153\end{array}$

$\begin{array}{llll}\mathrm{H} & 0.794177 & 2.039576 & 3.186032\end{array}$

$\begin{array}{llll}\mathrm{H} & 2.320270 & 1.580303 & 2.424503\end{array}$

H $\quad 1.431183 \quad 0.405772 \quad 3.401004$ 


\begin{tabular}{|c|c|c|c|}
\hline C & 4.351806 & -2.317459 & 0.403478 \\
\hline $\mathrm{H}$ & 3.801660 & -2.437834 & -0.539026 \\
\hline $\mathrm{H}$ & 4.261678 & -3.250395 & 0.973346 \\
\hline $\mathrm{H}$ & 5.408416 & -2.182920 & 0.142786 \\
\hline $\mathrm{C}$ & -5.187883 & -0.431018 & -0.083342 \\
\hline $\mathrm{H}$ & -5.900702 & -1.250347 & -0.084451 \\
\hline $\mathrm{C}$ & -3.377748 & -2.129852 & 0.387760 \\
\hline $\mathrm{H}$ & -2.507143 & -2.087385 & 1.058567 \\
\hline $\mathrm{C}$ & -4.732458 & 1.913282 & -0.295105 \\
\hline$H$ & -5.086064 & 2.925040 & -0.478341 \\
\hline$C$ & 1.512707 & 2.849641 & -1.128221 \\
\hline & 0.641748 & 2.244740 & -0.863578 \\
\hline & -2.424161 & 2.899203 & -0.187583 \\
\hline & -1.414438 & 2.559701 & 0.051162 \\
\hline & 5.078252 & 0.700903 & 0.039799 \\
\hline 11 & 5.986329 & 0.174906 & 0.324782 \\
\hline$C$ & -5.646409 & 0.865026 & -0.300173 \\
\hline $\mathrm{H}$ & -6.702348 & 1.053604 & -0.474812 \\
\hline C & 4.015846 & 2.567649 & -1.030256 \\
\hline $\mathrm{H}$ & 4.091639 & 3.498442 & -1.587503 \\
\hline $\mathrm{C}$ & -2.779583 & 4.021880 & 0.806878 \\
\hline $\mathrm{H}$ & -3.762912 & 4.458299 & 0.593551 \\
\hline $\mathrm{H}$ & -2.041145 & 4.830823 & 0.749806 \\
\hline & -2.798013 & 3.651749 & 1.837443 \\
\hline $\mathrm{C}$ & -2.399805 & 3.442152 & -1.631186 \\
\hline $\mathrm{H}$ & -2.096348 & 2.664264 & -2.340972 \\
\hline $\mathrm{H}$ & -1.694893 & 4.277969 & -1.719780 \\
\hline $\mathrm{H}$ & -3.387344 & 3.806676 & -1.938889 \\
\hline $\mathrm{C}$ & 4.581625 & -0.998381 & 2.549989 \\
\hline $\mathrm{H}$ & 5.650670 & -0.815661 & 2.388018 \\
\hline $\mathrm{H}$ & 4.486543 & -1.921925 & 3.133763 \\
\hline $\mathrm{H}$ & 4.189983 & -0.173664 & 3.154520 \\
\hline $\mathrm{C}$ & 1.405236 & 4.190625 & -0.375474 \\
\hline 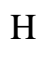 & 1.379439 & 4.033604 & 0.708317 \\
\hline & 0.492779 & 4.727319 & -0.662801 \\
\hline & 2.259109 & 4.842445 & -0.598286 \\
\hline 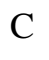 & 5.178437 & 1.890957 & -0.672786 \\
\hline 11 & 6.152755 & 2.288051 & -0.946100 \\
\hline & 1.473359 & 3.071524 & -2.652751 \\
\hline $\mathrm{H}$ & 2.302908 & 3.700716 & -2.996995 \\
\hline
\end{tabular}




$\begin{array}{llll}\mathrm{H} & 0.539736 & 3.566789 & -2.944432 \\ \mathrm{H} & 1.535051 & 2.119441 & -3.193597 \\ \mathrm{C} & -4.408119 & -3.029836 & 1.091081 \\ \mathrm{H} & -4.771821 & -2.564154 & 2.012621 \\ \mathrm{H} & -3.949071 & -3.989894 & 1.354436 \\ \mathrm{H} & -5.274635 & -3.245919 & 0.455223 \\ \mathrm{C} & -2.933284 & -2.790138 & -0.936437 \\ \mathrm{H} & -3.794456 & -2.947657 & -1.596562 \\ \mathrm{H} & -2.459825 & -3.766817 & -0.762557 \\ \mathrm{H} & -2.243646 & -2.148895 & -1.509223 \\ \mathrm{C} & 1.049364 & -2.347775 & -2.774855 \\ \mathrm{H} & 2.060546 & -2.008071 & -2.550306 \\ \mathrm{C} & 0.603152 & -3.554054 & -2.381796 \\ \mathrm{H} & -0.402219 & -3.856115 & -2.680752 \\ \mathrm{H} & 0.210483 & -2.879322 & 1.605155 \\ \mathrm{C} & 1.371064 & -4.549720 & -1.561679 \\ \mathrm{H} & 2.400157 & -4.225886 & -1.380682 \\ \mathrm{H} & 0.890937 & -4.721025 & -0.585646 \\ \mathrm{H} & 1.394761 & -5.521108 & -2.070982 \\ \mathrm{H} & 0.446533 & -1.694938 & -3.403024\end{array}$

\section{R-TS1-B}

Imaginary frequency: $-603.3797 \mathrm{~cm}^{-1}$

$\begin{array}{lccc}\text { Atom } & \mathrm{X} & \mathrm{Y} & \mathrm{Z} \\ \mathrm{Sm} & 0.067666 & -1.493153 & -0.120493 \\ \mathrm{~N} & 1.376218 & 0.326512 & 0.176194 \\ \mathrm{~N} & -1.532136 & 0.065688 & 0.298561 \\ \mathrm{C} & 2.640966 & 0.911393 & -0.114022 \\ \mathrm{C} & -2.900177 & 0.380392 & 0.183006 \\ \mathrm{C} & -0.759206 & 0.543239 & 1.389866 \\ \mathrm{C} & -3.838765 & -0.676128 & -0.034600 \\ \mathrm{C} & -1.450182 & 0.718975 & 2.737897 \\ \mathrm{H} & -0.953955 & 0.145367 & 3.529553 \\ \mathrm{H} & -2.487248 & 0.381119 & 2.678803 \\ \mathrm{H} & -1.467160 & 1.766119 & 3.065448 \\ \mathrm{C} & 3.855739 & 0.266836 & 0.253915 \\ \mathrm{C} & 0.630315 & 0.703344 & 1.317254 \\ \mathrm{C} & 2.696947 & 2.132439 & -0.839427 \\ \mathrm{C} & 3.893950 & -1.026686 & 1.063254\end{array}$




\begin{tabular}{|c|c|c|c|}
\hline $\mathrm{H}$ & 2.859419 & -1.317427 & 1.276425 \\
\hline $\mathrm{C}$ & -3.386235 & 1.720134 & 0.229115 \\
\hline $\mathrm{C}$ & 1.382002 & 1.153015 & 2.559173 \\
\hline $\mathrm{H}$ & 0.835597 & 1.925765 & 3.107743 \\
\hline $\mathrm{H}$ & 2.353270 & 1.568126 & 2.279177 \\
\hline $\mathrm{H}$ & 1.568216 & 0.325785 & 3.258671 \\
\hline $\mathrm{C}$ & 4.551582 & -2.176191 & 0.274482 \\
\hline $\mathrm{H}$ & 4.070450 & -2.321410 & -0.699485 \\
\hline $\mathrm{H}$ & 4.491544 & -3.118016 & 0.834809 \\
\hline $\mathrm{H}$ & 5.612058 & -1.974198 & 0.082940 \\
\hline C & -5.203442 & -0.387268 & -0.126013 \\
\hline $\mathrm{H}$ & -5.912663 & -1.195121 & -0.276755 \\
\hline $\mathrm{C}$ & -3.361303 & -2.109777 & -0.225309 \\
\hline $\mathrm{H}$ & -2.512698 & -2.264923 & 0.463381 \\
\hline $\mathrm{C}$ & -4.764104 & 1.951454 & 0.143206 \\
\hline $\mathrm{H}$ & -5.127321 & 2.975226 & 0.182340 \\
\hline C & 1.433006 & 2.875975 & -1.255724 \\
\hline $\mathrm{H}$ & 0.578418 & 2.312571 & -0.873406 \\
\hline $\mathrm{C}$ & -2.458530 & 2.933925 & 0.239655 \\
\hline $\mathrm{H}$ & -1.438461 & 2.590578 & 0.424739 \\
\hline $\mathrm{C}$ & 5.075959 & 0.841408 & -0.126688 \\
\hline $\mathrm{H}$ & 6.003774 & 0.348595 & 0.153959 \\
\hline $\mathrm{C}$ & -5.678096 & 0.916622 & -0.019370 \\
\hline $\mathrm{H}$ & -6.743077 & 1.122857 & -0.082851 \\
\hline $\mathrm{C}$ & 3.941502 & 2.665119 & -1.193900 \\
\hline $\mathrm{H}$ & 3.979645 & 3.595083 & -1.756196 \\
\hline $\mathrm{C}$ & -2.805232 & 3.961370 & 1.334846 \\
\hline $\mathrm{H}$ & -3.781759 & 4.429698 & 1.162876 \\
\hline $\mathrm{H}$ & -2.058461 & 4.764368 & 1.351642 \\
\hline $\mathrm{H}$ & -2.830471 & 3.500501 & 2.328036 \\
\hline $\mathrm{C}$ & -2.470977 & 3.607070 & -1.149468 \\
\hline $\mathrm{H}$ & -2.161268 & 2.903983 & -1.930630 \\
\hline $\mathrm{H}$ & -1.789827 & 4.466256 & -1.172734 \\
\hline $\mathrm{H}$ & -3.474340 & 3.968842 & -1.404910 \\
\hline $\mathrm{C}$ & 4.602142 & -0.845307 & 2.420898 \\
\hline $\mathrm{H}$ & 5.658534 & -0.582060 & 2.290118 \\
\hline $\mathrm{H}$ & 4.561071 & -1.774838 & 3.002168 \\
\hline $\mathrm{H}$ & 4.132963 & -0.053886 & 3.013632 \\
\hline $\mathrm{C}$ & 1.383222 & 4.288495 & -0.641079 \\
\hline $\mathrm{H}$ & 1.433120 & 4.245309 & 0.452737 \\
\hline
\end{tabular}




\begin{tabular}{llll}
$\mathrm{H}$ & 0.454241 & 4.799459 & -0.919298 \\
$\mathrm{H}$ & 2.217603 & 4.910379 & -0.987304 \\
$\mathrm{C}$ & 5.129615 & 2.029529 & -0.846985 \\
$\mathrm{H}$ & 6.087305 & 2.457031 & -1.132742 \\
$\mathrm{C}$ & 1.292319 & 2.937599 & -2.789448 \\
$\mathrm{H}$ & 2.112800 & 3.502120 & -3.249162 \\
$\mathrm{H}$ & 0.351758 & 3.426150 & -3.071505 \\
$\mathrm{H}$ & 1.297096 & 1.932084 & -3.226806 \\
$\mathrm{C}$ & -4.391428 & -3.195148 & 0.128656 \\
$\mathrm{H}$ & -4.798922 & -3.042219 & 1.133242 \\
$\mathrm{H}$ & -3.920673 & -4.185025 & 0.099356 \\
$\mathrm{H}$ & -5.228783 & -3.215023 & -0.578187 \\
$\mathrm{C}$ & -2.868687 & -2.312453 & -1.676343 \\
$\mathrm{H}$ & -3.696536 & -2.153879 & -2.376781 \\
$\mathrm{H}$ & -2.480111 & -3.324543 & -1.842946 \\
$\mathrm{H}$ & -2.100257 & -1.576002 & -1.962438 \\
$\mathrm{C}$ & 0.515992 & -3.963469 & -1.080371 \\
$\mathrm{C}$ & 0.892924 & -4.251549 & 0.220750 \\
$\mathrm{H}$ & 0.517751 & -2.965010 & 1.387459 \\
$\mathrm{H}$ & 1.938598 & -4.203475 & 0.515082 \\
$\mathrm{H}$ & 0.288489 & -4.919888 & 0.824381 \\
$\mathrm{H}$ & -0.472855 & -4.281450 & -1.410447 \\
$\mathrm{C}$ & 1.411192 & -3.351888 & -2.125997 \\
$\mathrm{H}$ & 1.304592 & -3.855721 & -3.093562 \\
$\mathrm{H}$ & 1.199236 & -2.274128 & -2.370471 \\
$\mathrm{H}$ & 2.467330 & -3.389870 & -1.840203 \\
& & & \\
\hline
\end{tabular}

\section{R-TS1-L}

Imaginary frequency: $-\mathbf{5 5 2 . 4 6 6} \mathrm{cm}^{-1}$

$\begin{array}{lccc}\text { Atom } & \mathrm{X} & \mathrm{Y} & \mathrm{Z} \\ \mathrm{Sm} & 0.017080 & -1.469381 & 0.115382 \\ \mathrm{~N} & -1.508754 & 0.120292 & -0.357186 \\ \mathrm{~N} & 1.410862 & 0.238584 & -0.351877 \\ \mathrm{C} & -2.869287 & 0.474375 & -0.190688 \\ \mathrm{C} & 2.757174 & 0.653365 & -0.179910 \\ \mathrm{C} & 0.636068 & 0.673770 & -1.457507 \\ \mathrm{C} & 3.827476 & -0.193679 & -0.579684 \\ \mathrm{C} & 1.357785 & 1.119378 & -2.720990 \\ \mathrm{H} & 0.869071 & 1.984205 & -3.180929\end{array}$




\begin{tabular}{|c|c|c|c|}
\hline $\mathrm{H}$ & 1.404978 & 0.333090 & -3.488749 \\
\hline $\mathrm{H}$ & 2.385992 & 1.405749 & -2.488500 \\
\hline $\mathrm{C}$ & -3.882035 & -0.516073 & -0.336376 \\
\hline $\mathrm{C}$ & -0.759933 & 0.598310 & -1.466767 \\
\hline $\mathrm{C}$ & -3.239417 & 1.795352 & 0.182912 \\
\hline $\mathrm{C}$ & -3.555946 & -1.949111 & -0.747828 \\
\hline & -2.532988 & -1.943032 & -1.154945 \\
\hline $\mathrm{C}$ & 3.046449 & 1.889288 & 0.457319 \\
\hline $\mathrm{C}$ & -1.511452 & 0.937621 & -2.748496 \\
\hline $\mathrm{H}$ & -1.519930 & 2.018720 & -2.943445 \\
\hline $\mathrm{H}$ & -2.553650 & 0.617398 & -2.676351 \\
\hline $\mathrm{H}$ & -1.073251 & 0.460761 & -3.632908 \\
\hline $\mathrm{C}$ & -3.579916 & -2.903754 & 0.463506 \\
\hline & -2.899152 & -2.568585 & 1.256517 \\
\hline & -3.293421 & -3.922645 & 0.175301 \\
\hline & -4.583949 & -2.943772 & 0.902154 \\
\hline & 5.146083 & 0.201810 & -0.321445 \\
\hline & 5.965805 & -0.447840 & -0.618694 \\
\hline C & 3.587321 & -1.528591 & -1.280133 \\
\hline $\mathrm{H}$ & 2.517998 & -1.577343 & -1.530680 \\
\hline $\mathrm{C}$ & 4.381081 & 2.246311 & 0.680386 \\
\hline $\mathrm{H}$ & 4.602839 & 3.192360 & 1.168377 \\
\hline $\mathrm{C}$ & -2.198889 & 2.889170 & 0.402952 \\
\hline $\mathrm{H}$ & -1.224829 & 2.484328 & 0.120808 \\
\hline$C$ & 1.933536 & 2.824358 & 0.916650 \\
\hline $\mathrm{H}$ & 0.985750 & 2.315725 & 0.722734 \\
\hline $\mathrm{C}$ & -5.216759 & -0.175449 & -0.082805 \\
\hline $\mathrm{H}$ & -5.989422 & -0.933481 & -0.182681 \\
\hline$c$ & 5.430023 & 1.413392 & 0.300847 \\
\hline $\mathrm{H}$ & 6.459909 & 1.705577 & 0.488787 \\
\hline $\mathrm{C}$ & -4.588318 & 2.088019 & 0.410121 \\
\hline $\mathrm{H}$ & -4.868801 & 3.096360 & 0.705026 \\
\hline $\mathrm{C}$ & 1.934052 & 4.143139 & 0.119092 \\
\hline $\mathrm{H}$ & 2.861643 & 4.707205 & 0.277357 \\
\hline $\mathrm{H}$ & 1.098797 & 4.783533 & 0.429168 \\
\hline $\mathrm{H}$ & 1.836711 & 3.957734 & -0.956309 \\
\hline , & 2.005578 & 3.098951 & 2.431278 \\
\hline 11 & 1.980620 & 2.164672 & 3.003838 \\
\hline $\mathrm{H}$ & 1.155931 & 3.714367 & 2.750589 \\
\hline 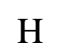 & 2.923120 & 3.633033 & 2.706483 \\
\hline
\end{tabular}




$\begin{array}{llll}\mathrm{C} & -4.462016 & -2.482266 & -1.873402 \\ \mathrm{H} & -5.501988 & -2.587203 & -1.544546 \\ \mathrm{H} & -4.122393 & -3.472982 & -2.199167 \\ \mathrm{H} & -4.451920 & -1.813847 & -2.741073 \\ \mathrm{C} & -2.456928 & 4.128933 & -0.474990 \\ \mathrm{H} & -2.504163 & 3.863201 & -1.536956 \\ \mathrm{H} & -1.653521 & 4.864420 & -0.345180 \\ \mathrm{H} & -3.400788 & 4.623077 & -0.214822 \\ \mathrm{C} & -5.577450 & 1.116229 & 0.286262 \\ \mathrm{H} & -6.618682 & 1.362712 & 0.476801 \\ \mathrm{C} & -2.119771 & 3.281215 & 1.892032 \\ \mathrm{H} & -3.067648 & 3.705114 & 2.245611 \\ \mathrm{H} & -1.337257 & 4.032986 & 2.053956 \\ \mathrm{H} & -1.889547 & 2.411031 & 2.516873 \\ \mathrm{C} & 4.352875 & -1.649141 & -2.611703 \\ \mathrm{H} & 4.115008 & -0.816218 & -3.281843 \\ \mathrm{H} & 4.091677 & -2.584733 & -3.121398 \\ \mathrm{H} & 5.437718 & -1.652776 & -2.456061 \\ \mathrm{C} & 3.908134 & -2.721417 & -0.357378 \\ \mathrm{H} & 4.966062 & -2.719763 & -0.069840 \\ \mathrm{H} & 3.696081 & -3.675664 & -0.855549 \\ \mathrm{H} & 3.321036 & -2.684464 & 0.568764 \\ \mathrm{C} & -0.043921 & -1.624967 & 2.771176 \\ \mathrm{H} & -1.017739 & -1.158708 & 2.917849 \\ \mathrm{C} & 0.074177 & -3.000859 & 2.666383 \\ \mathrm{H} & 0.826390 & -1.014636 & 3.015312 \\ \mathrm{H} & 0.121292 & -3.542076 & 0.852806 \\ \mathrm{H} & -0.833653 & -3.596009 & 2.717355 \\ \mathrm{H} & 1.341503 & -3.734480 & 3.034586 \\ & 1.407709 & -4.707603 & 2.543074 \\ & 1.355105 & -3.890801 & 4.122844\end{array}$

\section{Ph-TS1-B}

Imaginary frequency: $-\mathbf{- 4 8 8 . 2 9 8 3} \mathrm{cm}^{-1}$

$\begin{array}{lccc}\text { Atom } & \mathrm{X} & \mathrm{Y} & \mathrm{Z} \\ \mathrm{Sm} & -0.036987 & -1.241491 & -0.209556 \\ \mathrm{~N} & -1.614832 & 0.347455 & -0.471217 \\ \mathrm{~N} & 1.289797 & 0.543548 & -0.603578\end{array}$




\begin{tabular}{|c|c|c|c|}
\hline $\mathrm{C}$ & -2.971934 & 0.655705 & -0.201925 \\
\hline $\mathrm{C}$ & 2.617376 & 1.023402 & -0.423617 \\
\hline $\mathrm{C}$ & 0.450703 & 1.048166 & -1.627983 \\
\hline $\mathrm{C}$ & 3.727512 & 0.376119 & -1.036778 \\
\hline $\mathrm{C}$ & 1.090565 & 1.607286 & -2.887508 \\
\hline $\mathrm{H}$ & 1.175396 & 0.857164 & -3.687101 \\
\hline $\mathrm{H}$ & 2.099046 & 1.968254 & -2.671393 \\
\hline $\mathrm{H}$ & 0.518325 & 2.447237 & -3.292143 \\
\hline $\mathrm{C}$ & -3.991915 & -0.315434 & -0.417263 \\
\hline $\mathrm{C}$ & -0.940801 & 0.930761 & -1.576953 \\
\hline $\mathrm{C}$ & -3.332935 & 1.924279 & 0.332812 \\
\hline $\mathrm{C}$ & -3.711337 & -1.685666 & -1.028013 \\
\hline $\mathrm{H}$ & -2.650375 & -1.714201 & -1.308736 \\
\hline $\mathrm{C}$ & 2.850590 & 2.131834 & 0.435307 \\
\hline $\mathrm{C}$ & -1.762148 & 1.310712 & -2.802884 \\
\hline $\mathrm{H}$ & -2.805431 & 1.016354 & -2.668128 \\
\hline $\mathrm{H}$ & -1.394078 & 0.831143 & -3.718008 \\
\hline $\mathrm{H}$ & -1.753981 & 2.393888 & -2.984149 \\
\hline $\mathrm{C}$ & -3.966356 & -2.825448 & -0.022137 \\
\hline $\mathrm{H}$ & -3.391244 & -2.683742 & 0.901726 \\
\hline $\mathrm{H}$ & -3.695888 & -3.798136 & -0.452637 \\
\hline $\mathrm{H}$ & -5.023654 & -2.871063 & 0.264062 \\
\hline $\mathrm{C}$ & 5.022338 & 0.839063 & -0.766912 \\
\hline $\mathrm{H}$ & 5.870221 & 0.343182 & -1.233668 \\
\hline $\mathrm{C}$ & 3.569046 & -0.806656 & -1.989355 \\
\hline $\mathrm{H}$ & 2.497644 & -0.991161 & -2.118474 \\
\hline $\mathrm{C}$ & 4.162871 & 2.560022 & 0.666372 \\
\hline $\mathrm{H}$ & 4.336089 & 3.408677 & 1.324062 \\
\hline $\mathrm{C}$ & -2.291602 & 3.001207 & 0.618074 \\
\hline $\mathrm{H}$ & -1.324463 & 2.625618 & 0.278688 \\
\hline $\mathrm{C}$ & 1.702306 & 2.867371 & 1.115170 \\
\hline $\mathrm{H}$ & 0.784629 & 2.329227 & 0.865555 \\
\hline $\mathrm{C}$ & -5.316470 & -0.008924 & -0.077449 \\
\hline $\mathrm{H}$ & -6.091614 & -0.754369 & -0.239315 \\
\hline $\mathrm{C}$ & 5.249211 & 1.922444 & 0.075372 \\
\hline $\mathrm{H}$ & 6.262130 & 2.267314 & 0.267370 \\
\hline $\mathrm{C}$ & -4.671479 & 2.184205 & 0.644031 \\
\hline $\mathrm{H}$ & -4.939504 & 3.152923 & 1.059238 \\
\hline $\mathrm{C}$ & 1.565406 & 4.307856 & 0.584840 \\
\hline $\mathrm{H}$ & 2.461906 & 4.902085 & 0.801113 \\
\hline
\end{tabular}




\begin{tabular}{|c|c|c|c|}
\hline $\mathrm{H}$ & 0.710639 & 4.813915 & 1.050533 \\
\hline $\mathrm{H}$ & 1.414039 & 4.316179 & -0.500155 \\
\hline $\mathrm{C}$ & 1.841218 & 2.856937 & 2.649841 \\
\hline $\mathrm{H}$ & 1.915243 & 1.831784 & 3.031073 \\
\hline $\mathrm{H}$ & 0.970484 & 3.331354 & 3.118161 \\
\hline $\mathrm{H}$ & 2.733450 & 3.400940 & 2.983223 \\
\hline $\mathrm{C}$ & -4.506874 & -1.925418 & -2.326538 \\
\hline $\mathrm{H}$ & -5.587058 & -1.943841 & -2.140774 \\
\hline $\mathrm{H}$ & -4.229964 & -2.887708 & -2.774265 \\
\hline $\mathrm{H}$ & -4.308064 & -1.138748 & -3.062021 \\
\hline $\mathrm{C}$ & -2.579475 & 4.304804 & -0.152107 \\
\hline $\mathrm{H}$ & -2.646833 & 4.122360 & -1.230318 \\
\hline $\mathrm{H}$ & -1.780504 & 5.036453 & 0.018671 \\
\hline $\mathrm{H}$ & -3.522838 & 4.764387 & 0.166800 \\
\hline $\mathrm{C}$ & -5.665427 & 1.229260 & 0.449150 \\
\hline $\mathrm{H}$ & -6.698986 & 1.448225 & 0.704474 \\
\hline $\mathrm{C}$ & -2.178691 & 3.273880 & 2.131140 \\
\hline $\mathrm{H}$ & -3.121778 & 3.653437 & 2.542819 \\
\hline $\mathrm{H}$ & -1.402363 & 4.021900 & 2.334032 \\
\hline $\mathrm{H}$ & -1.920687 & 2.359572 & 2.677806 \\
\hline C & 4.153876 & -0.516961 & -3.386141 \\
\hline $\mathrm{H}$ & 3.712518 & 0.383046 & -3.825717 \\
\hline $\mathrm{H}$ & 3.958646 & -1.357265 & -4.063782 \\
\hline $\mathrm{H}$ & 5.239728 & -0.369580 & -3.345869 \\
\hline $\mathrm{C}$ & 4.190558 & -2.092997 & -1.411647 \\
\hline $\mathrm{H}$ & 5.275528 & -1.993208 & -1.287822 \\
\hline $\mathrm{H}$ & 4.007049 & -2.945831 & -2.077127 \\
\hline $\mathrm{H}$ & 3.770753 & -2.328252 & -0.426896 \\
\hline $\mathrm{C}$ & -0.194842 & -3.823420 & 0.827755 \\
\hline $\mathrm{C}$ & 0.329722 & -4.207113 & -0.388075 \\
\hline $\mathrm{H}$ & 1.397149 & -4.215354 & -0.583267 \\
\hline $\mathrm{H}$ & 0.203645 & -2.793113 & -1.725036 \\
\hline $\mathrm{H}$ & -0.257883 & -4.819032 & -1.061064 \\
\hline $\mathrm{H}$ & -1.261328 & -3.979980 & 0.988148 \\
\hline $\mathrm{C}$ & 0.490763 & -3.077328 & 1.881658 \\
\hline $\mathrm{C}$ & -0.280307 & -2.376823 & 2.846590 \\
\hline $\mathrm{C}$ & 1.897473 & -2.895937 & 1.925118 \\
\hline $\mathrm{C}$ & 0.323508 & -1.583120 & 3.825433 \\
\hline $\mathrm{H}$ & -1.361967 & -2.500183 & 2.848617 \\
\hline $\mathrm{C}$ & 2.490066 & -2.094241 & 2.899862 \\
\hline
\end{tabular}




$\begin{array}{llll}\mathrm{H} & 2.528031 & -3.426807 & 1.218288 \\ \mathrm{C} & 1.711158 & -1.436406 & 3.858514 \\ \mathrm{H} & -0.297777 & -1.077012 & 4.558977 \\ \mathrm{H} & 3.570988 & -1.987122 & 2.912981 \\ \mathrm{H} & 2.181424 & -0.816048 & 4.614997\end{array}$

\section{Ph-TS1-L}

Imaginary frequency: $-641.2036 \mathrm{~cm}^{-1}$

\begin{tabular}{lccc} 
Atom & \multicolumn{1}{c}{$\mathrm{X}$} & $\mathrm{Y}$ & $\mathrm{Z}$ \\
$\mathrm{Sm}$ & -0.123828 & -0.957095 & 0.534957 \\
$\mathrm{~N}$ & 1.954945 & -0.119453 & 0.339976 \\
$\mathrm{~N}$ & -0.620958 & 1.239888 & 0.532157 \\
$\mathrm{C}$ & 3.275413 & -0.440762 & -0.056487 \\
$\mathrm{C}$ & -1.723473 & 2.115467 & 0.351471 \\
$\mathrm{C}$ & 0.479128 & 1.585109 & 1.355947 \\
$\mathrm{C}$ & -2.906271 & 1.952744 & 1.124775 \\
$\mathrm{C}$ & 0.277031 & 2.635228 & 2.437761 \\
$\mathrm{H}$ & 1.159596 & 3.272329 & 2.550108 \\
$\mathrm{H}$ & 0.066287 & 2.199860 & 3.425764 \\
$\mathrm{H}$ & -0.567055 & 3.280861 & 2.183852 \\
$\mathrm{C}$ & 3.855603 & -1.675066 & 0.352323 \\
$\mathrm{C}$ & 1.706053 & 0.919852 & 1.277884 \\
$\mathrm{C}$ & 4.011930 & 0.424340 & -0.910566 \\
$\mathrm{C}$ & 3.121447 & -2.637984 & 1.281658 \\
$\mathrm{H}$ & 2.308136 & -2.067609 & 1.755728 \\
$\mathrm{C}$ & -1.680186 & 3.114197 & -0.657522 \\
$\mathrm{C}$ & 2.792066 & 1.239401 & 2.298322 \\
$\mathrm{H}$ & 3.271037 & 2.207578 & 2.097360 \\
$\mathrm{H}$ & 3.579204 & 0.482184 & 2.267900 \\
$\mathrm{H}$ & 2.407374 & 1.282764 & 3.323590 \\
$\mathrm{C}$ & 2.489513 & -3.807431 & 0.498846 \\
$\mathrm{H}$ & 1.809387 & -3.450527 & -0.285382 \\
$\mathrm{H}$ & 1.925858 & -4.475046 & 1.162556 \\
$\mathrm{H}$ & 3.265141 & -4.400445 & 0.000114 \\
$\mathrm{C}$ & -4.011563 & 2.772129 & 0.862719 \\
$\mathrm{H}$ & -4.919404 & 2.645661 & 1.447888 \\
$\mathrm{C}$ & -3.012406 & 0.916988 & 2.241729 \\
$\mathrm{H}$ & -2.020080 & 0.456607 & 2.353701 \\
$\mathrm{C}$ & -2.806652 & 3.916521 & -0.872334
\end{tabular}




\begin{tabular}{|c|c|c|c|}
\hline $\mathrm{H}$ & -2.773949 & 4.681585 & -1.644416 \\
\hline $\mathrm{C}$ & 3.426031 & 1.738138 & -1.417791 \\
\hline $\mathrm{H}$ & 2.453527 & 1.874322 & -0.939663 \\
\hline $\mathrm{C}$ & -0.437410 & 3.320133 & -1.515897 \\
\hline $\mathrm{H}$ & 0.276703 & 2.539966 & -1.240431 \\
\hline $\mathrm{C}$ & 5.128242 & -2.023641 & -0.117148 \\
\hline $\mathrm{H}$ & 5.568903 & -2.970405 & 0.184519 \\
\hline C & -3.969823 & 3.751039 & -0.125433 \\
\hline $\mathrm{H}$ & -4.836565 & 4.380080 & -0.311231 \\
\hline $\mathrm{C}$ & 5.286243 & 0.037280 & -1.338985 \\
\hline $\mathrm{H}$ & 5.845519 & 0.695874 & -1.999248 \\
\hline $\mathrm{C}$ & 0.224355 & 4.684239 & -1.238212 \\
\hline $\mathrm{H}$ & -0.443118 & 5.514773 & -1.499661 \\
\hline $\mathrm{H}$ & 1.141697 & 4.795761 & -1.829607 \\
\hline $\mathrm{H}$ & 0.488416 & 4.789729 & -0.180169 \\
\hline $\mathrm{C}$ & -0.741306 & 3.155018 & -3.017531 \\
\hline $\mathrm{H}$ & -1.182184 & 2.173540 & -3.227326 \\
\hline $\mathrm{H}$ & 0.179315 & 3.244685 & -3.606791 \\
\hline $\mathrm{H}$ & -1.441179 & 3.918062 & -3.379286 \\
\hline $\mathrm{C}$ & 4.000104 & -3.171423 & 2.428379 \\
\hline $\mathrm{H}$ & 4.799786 & -3.823908 & 2.060308 \\
\hline $\mathrm{H}$ & 3.396216 & -3.761657 & 3.128292 \\
\hline $\mathrm{H}$ & 4.466666 & -2.351892 & 2.985393 \\
\hline $\mathrm{C}$ & 4.300459 & 2.951298 & -1.046229 \\
\hline $\mathrm{H}$ & 4.471194 & 3.004588 & 0.034714 \\
\hline $\mathrm{H}$ & 3.814836 & 3.883768 & -1.359230 \\
\hline $\mathrm{H}$ & 5.281476 & 2.910535 & -1.535052 \\
\hline $\mathrm{C}$ & 5.847388 & -1.177759 & -0.955495 \\
\hline $\mathrm{H}$ & 6.835223 & -1.463347 & -1.307433 \\
\hline C & 3.185239 & 1.685652 & -2.939838 \\
\hline $\mathrm{H}$ & 4.124858 & 1.558530 & -3.491312 \\
\hline $\mathrm{H}$ & 2.717214 & 2.614448 & -3.289215 \\
\hline $\mathrm{H}$ & 2.526114 & 0.852024 & -3.206628 \\
\hline $\mathrm{C}$ & -3.354301 & 1.555451 & 3.602076 \\
\hline $\mathrm{H}$ & -2.630331 & 2.332133 & 3.870084 \\
\hline $\mathrm{H}$ & -3.352997 & 0.796819 & 4.394540 \\
\hline $\mathrm{H}$ & -4.348773 & 2.016106 & 3.589604 \\
\hline $\mathrm{C}$ & -4.015304 & -0.203614 & 1.901077 \\
\hline $\mathrm{H}$ & -5.030093 & 0.197774 & 1.794909 \\
\hline $\mathrm{H}$ & -4.039365 & -0.960936 & 2.694678 \\
\hline
\end{tabular}




$\begin{array}{llll}\mathrm{H} & -3.767287 & -0.705721 & 0.958459 \\ \mathrm{C} & -0.568330 & -1.794474 & -1.921710 \\ \mathrm{H} & 0.460268 & -1.943565 & -2.246104 \\ \mathrm{C} & -1.283775 & -2.875249 & -1.404570 \\ \mathrm{H} & -1.077077 & -0.937110 & -2.356336 \\ \mathrm{H} & -1.176920 & -2.889041 & 0.418858 \\ \mathrm{H} & -0.766243 & -3.827447 & -1.334507 \\ \mathrm{C} & -2.765213 & -3.006745 & -1.451253 \\ \mathrm{C} & -3.345709 & -4.257168 & -1.183505 \\ \mathrm{C} & -3.611625 & -1.940238 & -1.794004 \\ \mathrm{C} & -4.724471 & -4.440182 & -1.256210 \\ \mathrm{H} & -2.702578 & -5.089919 & -0.908619 \\ \mathrm{C} & -4.994131 & -2.119420 & -1.858641 \\ \mathrm{H} & -3.197097 & -0.962531 & -2.021838 \\ \mathrm{C} & -5.556638 & -3.368909 & -1.591472 \\ \mathrm{H} & -5.150466 & -5.418387 & -1.049469 \\ \mathrm{H} & -5.630777 & -1.279913 & -2.124650 \\ \mathrm{H} & -6.632904 & -3.507677 & -1.646389\end{array}$

\section{B}

$\begin{array}{llll}\text { Atom } & \mathrm{X} & \mathrm{Y} & \mathrm{Z}\end{array}$

$\begin{array}{llll}\text { Sm } & -0.025037 & -1.471963 & 0.250937\end{array}$

$\begin{array}{llll}\mathrm{N} & -1.488030 & 0.039589 & -0.515216\end{array}$

$\begin{array}{llll}\mathrm{N} & 1.338532 & 0.067413 & -0.642957\end{array}$

$\begin{array}{llll}\text { C } & -2.836515 & 0.385924 & -0.241864\end{array}$

$\begin{array}{llll}\text { C } & 2.700402 & 0.420570 & -0.487397\end{array}$

$\begin{array}{llll}\text { C } & 0.562908 & 0.506785 & -1.750027\end{array}$

$\begin{array}{llll}\text { C } & 3.695320 & -0.592027 & -0.605039\end{array}$

$\begin{array}{llll}\text { C } & 1.285305 & 0.854717 & -3.044923\end{array}$

$\begin{array}{llll}\mathrm{H} & 0.986745 & 0.209406 & -3.880802\end{array}$

$\begin{array}{llll}\mathrm{H} & 2.365280 & 0.747003 & -2.918217\end{array}$

H $\quad 1.100257 \quad 1.889974 \quad-3.358041$

$\begin{array}{llll}\text { C } & -3.853794 & -0.596059 & -0.400392\end{array}$

$\begin{array}{llll}\text { C } & -0.820531 & 0.500992 & -1.681966\end{array}$

$\begin{array}{llll}\text { C } & -3.178891 & 1.672300 & 0.251862\end{array}$

$\begin{array}{llll}\text { C } & -3.532104 & -1.994449 & -0.923081\end{array}$

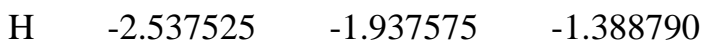

$\begin{array}{llll}\text { C } & 3.092933 & 1.744592 & -0.153079\end{array}$

$\begin{array}{llll}\text { C } & -1.671753 & 0.849272 & -2.885215\end{array}$

$\begin{array}{llll}\mathrm{H} & -1.107323 & 1.389263 & -3.647751\end{array}$ 


\begin{tabular}{|c|c|c|c|}
\hline $\mathrm{H}$ & -2.518353 & 1.478071 & -2.589897 \\
\hline $\mathrm{H}$ & -2.101016 & -0.044943 & -3.362783 \\
\hline $\mathrm{C}$ & -3.464310 & -3.025668 & 0.223994 \\
\hline & -2.757464 & -2.723556 & 1.011181 \\
\hline $\mathrm{H}$ & -3.175443 & -4.018946 & -0.145674 \\
\hline & -4.438633 & -3.125619 & 0.716115 \\
\hline C & 5.036039 & -0.271808 & -0.360437 \\
\hline $\mathrm{H}$ & 5.795091 & -1.046280 & -0.437766 \\
\hline $\mathrm{C}$ & 3.333311 & -2.025786 & -0.987969 \\
\hline 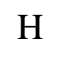 & 2.309180 & -1.996626 & -1.392101 \\
\hline C & 4.448069 & 2.018222 & 0.063438 \\
\hline & 4.748216 & 3.029621 & 0.326575 \\
\hline & -2.119853 & 2.742213 & 0.493841 \\
\hline & -1.156158 & 2.319444 & 0.200504 \\
\hline & 2.071486 & 2.862801 & 0.026534 \\
\hline & 1.086824 & 2.451439 & -0.206660 \\
\hline $\mathrm{C}$ & -5.173161 & -0.279522 & -0.056329 \\
\hline $\mathrm{H}$ & -5.951664 & -1.030159 & -0.167062 \\
\hline $\mathrm{C}$ & 5.418746 & 1.024512 & -0.029918 \\
\hline $\mathrm{H}$ & 6.464149 & 1.258038 & 0.153749 \\
\hline $\mathrm{C}$ & -4.515443 & 1.946769 & 0.564759 \\
\hline $\mathrm{H}$ & -4.779122 & 2.930484 & 0.946152 \\
\hline$C$ & 2.322470 & 4.039890 & -0.935758 \\
\hline $\mathrm{H}$ & 3.281901 & 4.531558 & -0.733571 \\
\hline $\mathrm{H}$ & 1.535318 & 4.796520 & -0.829326 \\
\hline $\mathrm{H}$ & 2.333593 & 3.707386 & -1.979602 \\
\hline $\mathrm{C}$ & 2.033708 & 3.349769 & 1.488851 \\
\hline $\mathrm{H}$ & 1.810945 & 2.524475 & 2.174234 \\
\hline $\mathrm{H}$ & 1.262094 & 4.118410 & 1.619449 \\
\hline $\mathrm{H}$ & 2.992869 & 3.786725 & 1.792518 \\
\hline$C$ & -4.500852 & -2.474682 & -2.019154 \\
\hline$H$ & -5.514814 & -2.624722 & -1.631573 \\
\hline 4 & -4.165885 & -3.433261 & -2.434255 \\
\hline & -4.559377 & -1.749365 & -2.837483 \\
\hline & -2.358740 & 4.001756 & -0.361044 \\
\hline & -2.385365 & 3.760804 & -1.429587 \\
\hline & -1.557006 & 4.733012 & -0.199704 \\
\hline 11 & -3.307291 & 4.489866 & -0.105594 \\
\hline & -5.510846 & 0.984763 & 0.418108 \\
\hline & -6.540986 & 1.216346 & 0.675934 \\
\hline
\end{tabular}




$\begin{array}{llll}\mathrm{C} & -2.027800 & 3.104156 & 1.989609 \\ \mathrm{H} & -2.961917 & 3.547668 & 2.355388 \\ \mathrm{H} & -1.224428 & 3.830543 & 2.162003 \\ \mathrm{H} & -1.818699 & 2.217187 & 2.598059 \\ \mathrm{C} & 4.219672 & -2.597771 & -2.110269 \\ \mathrm{H} & 4.212670 & -1.944145 & -2.988724 \\ \mathrm{H} & 3.860287 & -3.587932 & -2.416655 \\ \mathrm{H} & 5.260296 & -2.714405 & -1.787644 \\ \mathrm{C} & 3.346300 & -2.966312 & 0.236673 \\ \mathrm{H} & 4.354913 & -3.034765 & 0.660169 \\ \mathrm{H} & 3.031594 & -3.983469 & -0.034232 \\ \mathrm{H} & 2.701828 & -2.603091 & 1.050640 \\ \mathrm{C} & -0.003106 & -1.624822 & 2.724777 \\ \mathrm{H} & 0.004217 & -2.700497 & 3.001467 \\ \mathrm{H} & -0.988285 & -1.257552 & 3.071191 \\ \mathrm{C} & 0.997851 & -1.067174 & 5.048787 \\ \mathrm{H} & 1.054163 & -2.125984 & 5.332963 \\ \mathrm{H} & 1.802125 & -0.534648 & 5.574528 \\ \mathrm{H} & 0.039976 & -0.679231 & 5.417812 \\ \mathrm{C} & 1.098931 & -0.904652 & 3.523045 \\ \mathrm{H} & 1.084517 & 0.170057 & 3.286065 \\ \mathrm{H} & 2.091987 & -1.259466 & 3.203429\end{array}$

\section{R-TS2-L}

\section{Imaginary frequency: $-108.8093 \mathrm{~cm}^{-1}$}

$\begin{array}{lccc}\text { Atom } & \mathrm{X} & \mathrm{Y} & \mathrm{Z} \\ \mathrm{Sm} & 0.456895 & -0.133546 & 0.393520 \\ \mathrm{~N} & -1.238618 & -1.377638 & -0.378203 \\ \mathrm{~N} & -0.881486 & 1.494270 & -0.420168 \\ \mathrm{C} & -1.867484 & -2.650154 & -0.270931 \\ \mathrm{C} & -1.094797 & 2.901504 & -0.340564 \\ \mathrm{C} & -1.191100 & 0.769597 & -1.592551 \\ \mathrm{C} & -0.058648 & 3.822272 & -0.659733 \\ \mathrm{C} & -1.284953 & 1.501166 & -2.922122 \\ \mathrm{H} & -2.124550 & 1.134087 & -3.521081 \\ \mathrm{H} & -0.379668 & 1.389734 & -3.535195 \\ \mathrm{H} & -1.437766 & 2.569796 & -2.757671 \\ \mathrm{C} & -1.095361 & -3.844678 & -0.300206 \\ \mathrm{C} & -1.339985 & -0.623152 & -1.576619\end{array}$




\begin{tabular}{|c|c|c|c|}
\hline C & -3.273895 & -2.735959 & -0.083343 \\
\hline $\mathrm{C}$ & 0.411765 & -3.850519 & -0.538740 \\
\hline $\mathrm{H}$ & 0.716648 & -2.818966 & -0.759217 \\
\hline $\mathrm{C}$ & -2.345981 & 3.392897 & 0.118767 \\
\hline $\mathrm{C}$ & -1.553186 & -1.365724 & -2.887995 \\
\hline & -1.414447 & -2.440006 & -2.747904 \\
\hline & -0.863418 & -1.036247 & -3.672791 \\
\hline $\mathrm{H}$ & -2.570846 & -1.221518 & -3.276448 \\
\hline $\mathrm{C}$ & 1.189943 & -4.309103 & 0.709599 \\
\hline $\mathrm{H}$ & 0.945149 & -3.695887 & 1.584692 \\
\hline $\mathrm{H}$ & 2.271623 & -4.245296 & 0.540289 \\
\hline & 0.949555 & -5.348430 & 0.962194 \\
\hline . & -0.302800 & 5.195624 & -0.525958 \\
\hline & 0.487340 & 5.900822 & -0.771748 \\
\hline & 1.321570 & 3.385032 & -1.144537 \\
\hline & 1.325433 & 2.287424 & -1.196503 \\
\hline & -2.540607 & 4.774243 & 0.230483 \\
\hline & -3.500447 & 5.148579 & 0.577648 \\
\hline C & -4.154692 & -1.493124 & -0.019589 \\
\hline П & -3.520618 & -0.626079 & -0.215111 \\
\hline C & -3.481353 & 2.450637 & 0.498638 \\
\hline $\mathrm{H}$ & -3.092271 & 1.432321 & 0.421196 \\
\hline $\mathrm{C}$ & -1.737286 & -5.077033 & -0.117182 \\
\hline $\mathrm{H}$ & -1.147196 & -5.990019 & -0.132368 \\
\hline C & -1.532213 & 5.677572 & -0.090087 \\
\hline $\mathrm{H}$ & -1.701085 & 6.747226 & 0.002939 \\
\hline 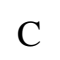 & -3.866822 & -3.991662 & 0.084789 \\
\hline & -4.941511 & -4.053850 & 0.237457 \\
\hline & -4.671088 & 2.579938 & -0.472465 \\
\hline $\mathrm{H}$ & -5.102511 & 3.588093 & -0.443785 \\
\hline $\mathrm{H}$ & -5.465621 & 1.870750 & -0.209552 \\
\hline $\mathrm{H}$ & -4.364731 & 2.378221 & -1.504889 \\
\hline $\mathrm{C}$ & -3.937142 & 2.659964 & 1.955898 \\
\hline $\mathrm{H}$ & -3.098098 & 2.551569 & 2.653094 \\
\hline $\mathrm{H}$ & -4.701523 & 1.922443 & 2.228242 \\
\hline & -4.369441 & 3.655949 & 2.109892 \\
\hline & 0.810177 & -4.694914 & -1.765353 \\
\hline & 0.572635 & -5.755015 & -1.620094 \\
\hline & 1.889309 & -4.620455 & -1.946818 \\
\hline & 0.288292 & -4.358338 & -2.667889 \\
\hline
\end{tabular}




\begin{tabular}{|c|c|c|c|}
\hline $\mathrm{C}$ & -5.259687 & -1.509818 & -1.093846 \\
\hline $\mathrm{H}$ & -4.836072 & -1.621319 & -2.098308 \\
\hline $\mathrm{H}$ & -5.832778 & -0.575119 & -1.070152 \\
\hline $\mathrm{H}$ & -5.966175 & -2.334209 & -0.938862 \\
\hline $\mathrm{C}$ & -3.111541 & -5.160591 & 0.074754 \\
\hline $\mathrm{H}$ & -3.589516 & -6.126615 & 0.214720 \\
\hline $\mathrm{C}$ & -4.759698 & -1.316225 & 1.387180 \\
\hline $\mathrm{H}$ & -5.417367 & -2.153871 & 1.649070 \\
\hline $\mathrm{H}$ & -5.354605 & -0.396299 & 1.441145 \\
\hline $\mathrm{H}$ & -3.974674 & -1.258879 & 2.149953 \\
\hline C & 1.635774 & 3.900823 & -2.563135 \\
\hline $\mathrm{H}$ & 0.876217 & 3.582054 & -3.283973 \\
\hline $\mathrm{H}$ & 2.609128 & 3.525468 & -2.901959 \\
\hline $\mathrm{H}$ & 1.677369 & 4.995991 & -2.586158 \\
\hline $\mathrm{C}$ & 2.431715 & 3.809265 & -0.162974 \\
\hline $\mathrm{H}$ & 2.513491 & 4.901080 & -0.109922 \\
\hline $\mathrm{H}$ & 3.404184 & 3.415892 & -0.481837 \\
\hline $\mathrm{H}$ & 2.233778 & 3.444308 & 0.850867 \\
\hline $\mathrm{C}$ & 0.206608 & 0.557403 & 4.641316 \\
\hline $\mathrm{H}$ & 0.786549 & 0.029318 & 5.406970 \\
\hline $\mathrm{H}$ & -0.082257 & 1.534515 & 5.047159 \\
\hline $\mathrm{H}$ & -0.709163 & -0.019288 & 4.463222 \\
\hline $\mathrm{C}$ & 1.029796 & 0.702344 & 3.352587 \\
\hline $\mathrm{H}$ & 0.426665 & 1.320039 & 2.647286 \\
\hline $\mathrm{H}$ & 1.919176 & 1.316165 & 3.551665 \\
\hline $\mathrm{C}$ & 4.960455 & -0.512097 & 0.277033 \\
\hline $\mathrm{C}$ & 4.970040 & -0.997376 & -1.041226 \\
\hline $\mathrm{C}$ & 6.186930 & -0.096727 & 0.823497 \\
\hline $\mathrm{C}$ & 6.144614 & -1.046596 & -1.794642 \\
\hline $\mathrm{H}$ & 4.041632 & -1.354557 & -1.488364 \\
\hline $\mathrm{C}$ & 7.369023 & -0.140917 & 0.080662 \\
\hline $\mathrm{H}$ & 6.220498 & 0.265151 & 1.850501 \\
\hline $\mathrm{C}$ & 7.348817 & -0.615198 & -1.232754 \\
\hline $\mathrm{H}$ & 6.124651 & -1.427485 & -2.813091 \\
\hline $\mathrm{H}$ & 8.305024 & 0.188012 & 0.526003 \\
\hline $\mathrm{H}$ & 8.267446 & -0.656464 & -1.812703 \\
\hline $\mathrm{H}$ & 2.581056 & -1.345563 & 0.374019 \\
\hline $\mathrm{C}$ & 1.491764 & -0.615307 & 2.712390 \\
\hline $\mathrm{H}$ & 2.098211 & -1.171845 & 3.432281 \\
\hline $\mathrm{H}$ & 0.614263 & -1.298102 & 2.570164 \\
\hline
\end{tabular}




$\begin{array}{llll}\mathrm{Si} & 3.348002 & -0.358394 & 1.304330 \\ \mathrm{H} & 3.992902 & -0.229845 & 2.652872 \\ \mathrm{H} & 2.769295 & 0.965041 & 0.842324\end{array}$

$\begin{array}{lccc}\text { B' } & & & \\ \text { Atom } & \mathrm{X} & \mathrm{Y} & \mathrm{Z} \\ \mathrm{Sm} & 0.144521 & -0.547155 & -0.712075 \\ \mathrm{~N} & -1.905304 & 0.303922 & -0.539419 \\ \mathrm{~N} & 0.657372 & 1.613803 & -0.456659 \\ \mathrm{C} & -3.223294 & -0.184938 & -0.327298 \\ \mathrm{C} & 1.689052 & 2.498131 & -0.041867 \\ \mathrm{C} & -0.386106 & 2.068696 & -1.304075 \\ \mathrm{C} & 2.986739 & 2.400812 & -0.614095 \\ \mathrm{C} & -0.109682 & 3.234098 & -2.242586 \\ \mathrm{H} & -0.617667 & 4.150568 & -1.911833 \\ \mathrm{H} & -0.443233 & 3.036383 & -3.267364 \\ \mathrm{H} & 0.958985 & 3.456114 & -2.273371 \\ \mathrm{C} & -3.759937 & -1.185673 & -1.182411 \\ \mathrm{C} & -1.636202 & 1.445356 & -1.333874 \\ \mathrm{C} & -3.975913 & 0.266756 & 0.787223 \\ \mathrm{C} & -2.992703 & -1.704072 & -2.397826 \\ \mathrm{H} & -2.149088 & -1.018978 & -2.568534 \\ \mathrm{C} & 1.444107 & 3.451731 & 0.981239 \\ \mathrm{C} & -2.698009 & 1.941076 & -2.301690 \\ \mathrm{H} & -3.683035 & 1.571214 & -2.007385 \\ \mathrm{H} & -2.523872 & 1.615245 & -3.337382 \\ \mathrm{H} & -2.745764 & 3.034521 & -2.314519 \\ \mathrm{C} & -2.416977 & -3.114797 & -2.151538 \\ \mathrm{H} & -1.759945 & -3.146512 & -1.272007 \\ \mathrm{H} & -1.843294 & -3.464640 & -3.019674 \\ \mathrm{H} & -3.221644 & -3.836805 & -1.971397 \\ \mathrm{C} & 4.000484 & 3.249439 & -0.150239 \\ \mathrm{H} & 4.994937 & 3.176332 & -0.583773 \\ \mathrm{C} & 3.325885 & 1.411301 & -1.726921 \\ \mathrm{H} & 2.394846 & 0.886933 & -1.992030 \\ \mathrm{C} & 2.488888 & 4.279152 & 1.405778 \\ \mathrm{H} & 2.304355 & 5.003098 & 2.195642 \\ \mathrm{C} & -3.434191 & 1.337329 & 1.726673 \\ & -2.401282 & 1.532130 & 1.426295 \\ 0.083451 & 3.562850 & 1.660712\end{array}$




\begin{tabular}{|c|c|c|c|}
\hline $\mathrm{H}$ & -0.593224 & 2.867266 & 1.159271 \\
\hline $\mathrm{C}$ & -5.021946 & -1.720699 & -0.897061 \\
\hline & -5.433921 & -2.494064 & -1.540318 \\
\hline & 3.761760 & 4.186220 & 0.849529 \\
\hline & 4.561921 & 4.835239 & 1.195630 \\
\hline$C$ & -5.238587 & -0.289698 & 1.022823 \\
\hline $\mathrm{A}$ & -5.819437 & 0.054343 & 1.874919 \\
\hline C & -0.520258 & 4.974532 & 1.530295 \\
\hline $\mathrm{H}$ & 0.084691 & 5.725907 & 2.052286 \\
\hline $\mathrm{H}$ & -1.526985 & 5.002232 & 1.964820 \\
\hline $\mathrm{H}$ & -0.596287 & 5.280986 & 0.480792 \\
\hline $\mathrm{C}$ & 0.167081 & 3.134829 & 3.139347 \\
\hline & 0.548060 & 2.111867 & 3.232269 \\
\hline $\boldsymbol{H}$ & -0.823515 & 3.173798 & 3.609648 \\
\hline 11 & 0.832855 & 3.792894 & 3.710965 \\
\hline$C$ & -3.832050 & -1.683420 & -3.689581 \\
\hline $\boldsymbol{H}$ & -4.671455 & -2.385625 & -3.637187 \\
\hline $\mathrm{H}$ & -3.217231 & -1.973055 & -4.550397 \\
\hline $\mathrm{H}$ & -4.241257 & -0.685965 & -3.880819 \\
\hline C & -4.220940 & 2.655822 & 1.590033 \\
\hline $\mathrm{H}$ & -4.201904 & 3.025112 & 0.558672 \\
\hline $\mathrm{H}$ & -3.789950 & 3.431231 & 2.235362 \\
\hline $\mathrm{H}$ & -5.271510 & 2.527761 & 1.879149 \\
\hline$C$ & -5.762649 & -1.278872 & 0.195418 \\
\hline 11 & -6.741799 & -1.703694 & 0.400323 \\
\hline 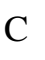 & -3.404718 & 0.863059 & 3.191993 \\
\hline H & -4.412122 & 0.675758 & 3.583184 \\
\hline $\mathrm{H}$ & -2.940464 & 1.624842 & 3.829675 \\
\hline $\mathrm{H}$ & -2.827933 & -0.062727 & 3.297254 \\
\hline $\mathrm{C}$ & 3.812516 & 2.115751 & -3.008834 \\
\hline $\mathrm{H}$ & 3.079570 & 2.845946 & -3.368947 \\
\hline $\mathrm{H}$ & 3.988203 & 1.384622 & -3.807767 \\
\hline $\mathrm{H}$ & 4.754905 & 2.648448 & -2.837230 \\
\hline $\mathrm{C}$ & 4.347721 & 0.353456 & -1.267053 \\
\hline $\mathrm{H}$ & 5.305284 & 0.821150 & -1.010122 \\
\hline$\Pi$ & 4.540144 & -0.378551 & -2.061272 \\
\hline П & 4.004251 & -0.190273 & -0.380419 \\
\hline C & -2.240625 & -4.232640 & 2.648214 \\
\hline $\mathbf{H}$ & -1.971575 & -4.731372 & 3.587445 \\
\hline$H$ & -3.238604 & -3.795636 & 2.771571 \\
\hline
\end{tabular}




$\begin{array}{llll}\mathrm{H} & -2.304109 & -5.003674 & 1.870418 \\ \mathrm{C} & -1.203772 & -3.164454 & 2.278860 \\ \mathrm{H} & -1.536949 & -2.658118 & 1.358627 \\ \mathrm{H} & -1.187013 & -2.392670 & 3.061323 \\ \mathrm{C} & 3.210303 & -2.877680 & 0.939725 \\ \mathrm{C} & 3.389824 & -3.565678 & -0.274674 \\ \mathrm{C} & 4.361121 & -2.576837 & 1.692654 \\ \mathrm{C} & 4.659286 & -3.939118 & -0.718884 \\ \mathrm{H} & 2.517507 & -3.814850 & -0.874988 \\ \mathrm{C} & 5.636590 & -2.931518 & 1.246055 \\ \mathrm{H} & 4.254205 & -2.057873 & 2.642042 \\ \mathrm{C} & 5.788049 & -3.615545 & 0.038808 \\ \mathrm{H} & 4.769795 & -4.481282 & -1.655194 \\ \mathrm{H} & 6.509896 & -2.677535 & 1.841901 \\ \mathrm{H} & 6.778718 & -3.897877 & -0.309070 \\ \mathrm{H} & 0.869083 & -2.540569 & -0.124601 \\ \mathrm{C} & 0.213764 & -3.729967 & 2.074011 \\ \mathrm{H} & 0.544047 & -4.225194 & 2.998436 \\ \mathrm{H} & 0.195619 & -4.491830 & 1.283636 \\ \mathrm{Si} & 1.506362 & -2.398534 & 1.639350 \\ \mathrm{H} & 1.956970 & -1.962199 & 3.025506 \\ \mathrm{H} & 0.945011 & -0.950113 & 1.387529\end{array}$

$\begin{array}{lccc}\text { 4' } & & & \\ \text { Atom } & \mathrm{X} & \mathrm{Y} & \mathrm{Z} \\ \mathrm{N} & -2.076784 & 0.129457 & -0.157041 \\ \mathrm{~N} & 0.649339 & 0.378117 & -1.150252 \\ \mathrm{C} & -3.281344 & 0.431140 & 0.500771 \\ \mathrm{C} & 1.928237 & 0.944694 & -1.367730 \\ \mathrm{C} & -0.472866 & 0.721719 & -1.936218 \\ \mathrm{C} & 2.956397 & 0.197726 & -2.012021 \\ \mathrm{C} & -0.241839 & 1.073376 & -3.397936 \\ \mathrm{H} & -0.327819 & 0.196325 & -4.056330 \\ \mathrm{H} & 0.764893 & 1.479014 & -3.529224 \\ \mathrm{H} & -0.953254 & 1.820978 & -3.761014 \\ \mathrm{C} & -4.003843 & -0.634285 & 1.128666 \\ \mathrm{C} & -1.785403 & 0.582604 & -1.467732 \\ \mathrm{C} & -3.785463 & 1.758871 & 0.627409 \\ \mathrm{C} & -3.467195 & -2.059033 & 1.069808 \\ \mathrm{H} & -3.064077 & -2.205538 & 0.055541\end{array}$




\begin{tabular}{|c|c|c|c|}
\hline C & 2.226352 & 2.243450 & -0.867913 \\
\hline $\mathrm{C}$ & -2.940611 & 0.725978 & -2.452976 \\
\hline $\mathrm{H}$ & -3.044462 & 1.752351 & -2.826488 \\
\hline $\mathrm{H}$ & -3.883715 & 0.457792 & -1.971308 \\
\hline $\mathrm{H}$ & -2.816288 & 0.079181 & -3.330196 \\
\hline $\mathrm{C}$ & -2.318602 & -2.256774 & 2.089189 \\
\hline $\mathrm{H}$ & -1.607382 & -1.414852 & 2.088160 \\
\hline $\mathrm{H}$ & -1.782292 & -3.204463 & 1.928904 \\
\hline $\mathrm{H}$ & -2.716889 & -2.281745 & 3.110092 \\
\hline $\mathrm{C}$ & -1.015083 & -4.101119 & -2.613729 \\
\hline $\mathrm{H}$ & -0.565700 & -3.801524 & -3.574311 \\
\hline $\mathrm{H}$ & -2.102014 & -4.193014 & -2.771182 \\
\hline $\mathrm{H}$ & -0.631696 & -5.105044 & -2.371057 \\
\hline & 4.246908 & 0.738794 & -2.096138 \\
\hline & 5.033129 & 0.163493 & -2.579661 \\
\hline & 2.697485 & -1.164740 & -2.650643 \\
\hline & 1.625435 & -1.370890 & -2.564485 \\
\hline$C$ & 3.524499 & 2.749019 & -1.000963 \\
\hline $\mathrm{H}$ & 3.746688 & 3.744448 & -0.623778 \\
\hline $\mathrm{C}$ & -3.009514 & 2.985775 & 0.149223 \\
\hline $\mathrm{H}$ & -2.162303 & 2.647888 & -0.450904 \\
\hline C & 1.160385 & 3.097924 & -0.190803 \\
\hline $\mathrm{H}$ & 0.280395 & 2.465272 & -0.049210 \\
\hline $\mathrm{C}$ & -5.193038 & -0.363366 & 1.809418 \\
\hline $\mathrm{H}$ & -5.742043 & -1.176497 & 2.274271 \\
\hline$C$ & 4.539692 & 2.004288 & -1.597147 \\
\hline $\mathrm{H}$ & 5.544011 & 2.411313 & -1.684135 \\
\hline . & -4.989648 & 1.970717 & 1.310041 \\
\hline & -5.367433 & 2.985899 & 1.403891 \\
\hline $\mathrm{C}$ & 0.753157 & 4.286012 & -1.085586 \\
\hline $\mathrm{H}$ & 1.603776 & 4.954402 & -1.269306 \\
\hline $\mathrm{H}$ & -0.039153 & 4.877202 & -0.610462 \\
\hline $\mathrm{H}$ & 0.383361 & 3.943227 & -2.058057 \\
\hline $\mathrm{C}$ & 1.599660 & 3.590801 & 1.201527 \\
\hline $\mathrm{H}$ & 1.901918 & 2.754758 & 1.842020 \\
\hline $\mathrm{H}$ & 0.774868 & 4.117757 & 1.695395 \\
\hline & 2.444588 & 4.288055 & 1.142303 \\
\hline & -4.514609 & -3.166753 & 1.265013 \\
\hline 11 & -4.913220 & -3.181983 & 2.286015 \\
\hline & -4.062244 & -4.147667 & 1.076961 \\
\hline
\end{tabular}




$\begin{array}{llll}\mathrm{H} & -5.354557 & -3.042609 & 0.573912 \\ \mathrm{C} & -3.851251 & 3.929084 & -0.732108 \\ \mathrm{H} & -4.290831 & 3.398193 & -1.583246 \\ \mathrm{H} & -3.227554 & 4.742258 & -1.123173 \\ \mathrm{H} & -4.671818 & 4.390216 & -0.169741 \\ \mathrm{C} & -5.701757 & 0.929991 & 1.895087 \\ \mathrm{H} & -6.632489 & 1.122920 & 2.421699 \\ \mathrm{C} & -2.432553 & 3.749690 & 1.359468 \\ \mathrm{H} & -3.231758 & 4.114531 & 2.015934 \\ \mathrm{H} & -1.847619 & 4.617391 & 1.029764 \\ \mathrm{H} & -1.778791 & 3.104040 & 1.956183 \\ \mathrm{C} & 3.031868 & -1.170539 & -4.155620 \\ \mathrm{H} & 2.484571 & -0.383420 & -4.685209 \\ \mathrm{H} & 2.761630 & -2.134525 & -4.603844 \\ \mathrm{H} & 4.101688 & -1.010343 & -4.333770 \\ \mathrm{C} & 3.447004 & -2.301139 & -1.929355 \\ \mathrm{H} & 4.533086 & -2.156303 & -1.975468 \\ \mathrm{H} & 3.217463 & -3.272009 & -2.384922 \\ \mathrm{H} & 3.171242 & -2.356331 & -0.868761 \\ \mathrm{Sm} & -0.449489 & -1.459881 & -0.363026 \\ \mathrm{C} & 3.240495 & -1.073765 & 2.979391 \\ \mathrm{C} & 3.804370 & -0.148351 & 2.079624 \\ \mathrm{C} & 3.383297 & -0.839340 & 4.359676 \\ \mathrm{C} & 4.488218 & 0.974117 & 2.547288 \\ \mathrm{H} & 3.712228 & -0.290233 & 1.005256 \\ \mathrm{C} & 4.072165 & 0.281119 & 4.827349 \\ \mathrm{H} & 2.953060 & -1.533705 & 5.077815 \\ \mathrm{C} & 4.623956 & 1.188472 & 3.921338 \\ \mathrm{H} & 4.904577 & 1.679104 & 1.834049 \\ \mathrm{H} & 4.173241 & 0.446756 & 5.896562 \\ \mathrm{H} & 5.155956 & 2.063651 & 4.284784 \\ \mathrm{O} & -0.715743 & -3.190459 & -1.598010 \\ \mathrm{Si} & 2.338068 & -2.591909 & 2.379491 \\ \mathrm{H} & 3.176233 & -3.538284 & 1.602034 \\ 1.682134 & -3.302651 & 3.505680 \\ & 1.223495 & -2.201732 & 1.437889\end{array}$

TS3

Imaginary frequency: $-\mathbf{- 9 7 4 . 5 2 6 6} \mathrm{cm}^{-1}$ 


\begin{tabular}{|c|c|c|c|}
\hline Atom & $X$ & $\mathrm{Y}$ & $\mathrm{Z}$ \\
\hline $\mathrm{N}$ & 2.067804 & -0.632074 & 0.194585 \\
\hline $\mathrm{N}$ & 0.094058 & 1.462113 & 0.330530 \\
\hline $\mathrm{C}$ & 3.255392 & -1.317131 & -0.144109 \\
\hline $\mathrm{C}$ & -0.555715 & 2.705250 & 0.084665 \\
\hline $\mathrm{C}$ & 1.077926 & 1.313125 & 1.332877 \\
\hline $\mathrm{C}$ & -1.830406 & 2.986460 & 0.648293 \\
\hline $\mathrm{C}$ & 1.003252 & 2.199702 & 2.563437 \\
\hline $\mathrm{H}$ & 0.394741 & 1.756622 & 3.365280 \\
\hline $\mathrm{H}$ & 0.545452 & 3.157936 & 2.304049 \\
\hline $\mathrm{H}$ & 1.993243 & 2.403388 & 2.980534 \\
\hline $\mathrm{C}$ & 3.209622 & -2.737280 & -0.297534 \\
\hline $\mathrm{C}$ & 2.017344 & 0.277956 & 1.282813 \\
\hline $\mathrm{C}$ & 4.485851 & -0.641651 & -0.383785 \\
\hline $\mathrm{C}$ & 1.890487 & -3.482270 & -0.146339 \\
\hline $\mathrm{H}$ & 1.354376 & -3.016525 & 0.696880 \\
\hline $\mathrm{C}$ & 0.046054 & 3.649352 & -0.788888 \\
\hline $\mathrm{C}$ & 2.894038 & 0.001844 & 2.498360 \\
\hline $\mathrm{H}$ & 3.632695 & 0.795429 & 2.666685 \\
\hline $\mathrm{H}$ & 3.445685 & -0.931482 & 2.364100 \\
\hline $\mathrm{H}$ & 2.305421 & -0.084981 & 3.419745 \\
\hline $\mathrm{C}$ & 1.033740 & -3.325289 & -1.423848 \\
\hline $\mathrm{H}$ & 0.991678 & -2.281462 & -1.776897 \\
\hline $\mathrm{H}$ & 0.014493 & -3.711560 & -1.293632 \\
\hline $\mathrm{H}$ & 1.489488 & -3.878529 & -2.253191 \\
\hline $\mathrm{C}$ & -1.666388 & -2.406622 & 3.168321 \\
\hline $\mathrm{H}$ & -1.912599 & -1.579417 & 3.844120 \\
\hline $\mathrm{H}$ & -0.858113 & -2.999816 & 3.611502 \\
\hline $\mathrm{H}$ & -2.552828 & -3.047566 & 3.063637 \\
\hline $\mathrm{C}$ & -2.476334 & 4.181882 & 0.306991 \\
\hline $\mathrm{H}$ & -3.455042 & 4.395315 & 0.729475 \\
\hline $\mathrm{C}$ & -2.532172 & 2.034670 & 1.612979 \\
\hline $\mathrm{H}$ & -1.835704 & 1.218904 & 1.839926 \\
\hline $\mathrm{C}$ & -0.637625 & 4.832364 & -1.092012 \\
\hline $\mathrm{H}$ & -0.179368 & 5.552506 & -1.765560 \\
\hline $\mathrm{C}$ & 4.597594 & 0.881368 & -0.429337 \\
\hline $\mathrm{H}$ & 3.665741 & 1.308634 & -0.052942 \\
\hline $\mathrm{C}$ & 1.414247 & 3.403997 & -1.414415 \\
\hline $\mathrm{H}$ & 1.768506 & 2.432740 & -1.059373 \\
\hline $\mathrm{C}$ & 4.373955 & -3.437212 & -0.624141 \\
\hline
\end{tabular}




\begin{tabular}{|c|c|c|c|}
\hline $\mathrm{H}$ & 4.338752 & -4.516976 & -0.728840 \\
\hline $\mathrm{C}$ & -1.893102 & 5.102993 & -0.556402 \\
\hline $\mathrm{H}$ & -2.411813 & 6.024305 & -0.808500 \\
\hline C & 5.625065 & -1.393929 & -0.696434 \\
\hline $\mathrm{H}$ & 6.563289 & -0.875836 & -0.877754 \\
\hline $\mathrm{C}$ & 2.434382 & 4.471063 & -0.971140 \\
\hline $\mathrm{H}$ & 2.140265 & 5.473514 & -1.304998 \\
\hline $\mathrm{H}$ & 3.424120 & 4.259660 & -1.392930 \\
\hline $\mathrm{H}$ & 2.529199 & 4.499641 & 0.120256 \\
\hline $\mathrm{C}$ & 1.330202 & 3.326727 & -2.951517 \\
\hline $\mathrm{H}$ & 0.625084 & 2.549839 & -3.269774 \\
\hline $\mathrm{H}$ & 2.311790 & 3.090590 & -3.379583 \\
\hline $\mathrm{H}$ & 0.996097 & 4.275851 & -3.387971 \\
\hline $\mathrm{C}$ & 2.012086 & -4.972788 & 0.208567 \\
\hline $\mathrm{H}$ & 2.436988 & -5.557622 & -0.615077 \\
\hline $\mathrm{H}$ & 1.020473 & -5.387985 & 0.422791 \\
\hline $\mathrm{H}$ & 2.642187 & -5.121213 & 1.091704 \\
\hline $\mathrm{C}$ & 5.742419 & 1.433605 & 0.442090 \\
\hline $\mathrm{H}$ & 5.661989 & 1.090847 & 1.479303 \\
\hline $\mathrm{H}$ & 5.723091 & 2.530062 & 0.444009 \\
\hline $\mathrm{H}$ & 6.726112 & 1.126475 & 0.067717 \\
\hline $\mathrm{C}$ & 5.586457 & -2.778760 & -0.809627 \\
\hline $\mathrm{H}$ & 6.484847 & -3.338380 & -1.055447 \\
\hline $\mathrm{C}$ & 4.759178 & 1.350636 & -1.890527 \\
\hline $\mathrm{H}$ & 5.680226 & 0.954141 & -2.334356 \\
\hline $\mathrm{H}$ & 4.805748 & 2.445059 & -1.944807 \\
\hline $\mathrm{H}$ & 3.919320 & 1.013556 & -2.507963 \\
\hline $\mathrm{C}$ & -2.876882 & 2.708481 & 2.955542 \\
\hline $\mathrm{H}$ & -1.985459 & 3.133819 & 3.429059 \\
\hline $\mathrm{H}$ & -3.316400 & 1.979205 & 3.647092 \\
\hline $\mathrm{H}$ & -3.603960 & 3.518550 & 2.826999 \\
\hline $\mathrm{C}$ & -3.790735 & 1.410720 & 0.979864 \\
\hline $\mathrm{H}$ & -4.538754 & 2.178136 & 0.748268 \\
\hline $\mathrm{H}$ & -4.254793 & 0.685598 & 1.659019 \\
\hline $\mathrm{H}$ & -3.561382 & 0.892134 & 0.041793 \\
\hline $\mathrm{Sm}$ & -0.183976 & -0.762289 & 0.182290 \\
\hline $\mathrm{C}$ & -4.591168 & -1.890915 & -1.071080 \\
\hline $\mathrm{C}$ & -5.612952 & -2.214514 & -0.158291 \\
\hline $\mathrm{C}$ & -4.951056 & -1.151476 & -2.214242 \\
\hline $\mathrm{C}$ & -6.937382 & -1.831825 & -0.381211 \\
\hline
\end{tabular}




$\begin{array}{llll}\mathrm{H} & -5.371840 & -2.773529 & 0.744207 \\ \mathrm{C} & -6.271334 & -0.755803 & -2.436607 \\ \mathrm{H} & -4.189975 & -0.882643 & -2.942876 \\ \mathrm{C} & -7.269237 & -1.098235 & -1.521498 \\ \mathrm{H} & -7.707515 & -2.099821 & 0.338056 \\ \mathrm{H} & -6.521638 & -0.182743 & -3.326105 \\ \mathrm{H} & -8.297865 & -0.792333 & -1.694695 \\ \mathrm{O} & -1.280558 & -1.914917 & 1.895350 \\ \mathrm{Si} & -2.811726 & -2.461245 & -0.733753 \\ \mathrm{H} & -2.012812 & -2.275044 & 1.023520 \\ \mathrm{H} & -2.579229 & -3.713978 & -1.533055 \\ \mathrm{H} & -1.982716 & -1.483589 & -1.622358\end{array}$

C

$\begin{array}{lccc}\text { Atom } & \mathrm{X} & \mathrm{Y} & \mathrm{Z} \\ \mathrm{N} & -1.669325 & -0.303462 & 0.388729 \\ \mathrm{~N} & 1.071701 & -1.198322 & 0.368447 \\ \mathrm{C} & -3.081472 & -0.298724 & 0.230899 \\ \mathrm{C} & 2.204900 & -2.024449 & 0.109855 \\ \mathrm{C} & 0.232125 & -1.404769 & 1.494261 \\ \mathrm{C} & 3.496546 & -1.670108 & 0.591630 \\ \mathrm{C} & 0.828861 & -2.031186 & 2.741279 \\ \mathrm{H} & 1.263265 & -1.291485 & 3.431786 \\ \mathrm{H} & 1.632723 & -2.720072 & 2.467556 \\ \mathrm{H} & 0.084391 & -2.600542 & 3.304251 \\ \mathrm{C} & -3.809794 & 0.919232 & 0.353501 \\ \mathrm{C} & -1.078902 & -0.943493 & 1.514650 \\ \mathrm{C} & -3.788670 & -1.491893 & -0.085860 \\ \mathrm{C} & -3.138911 & 2.242696 & 0.707367 \\ \mathrm{H} & -2.106001 & 2.015833 & 1.000548 \\ \mathrm{C} & 2.063287 & -3.182519 & -0.700756 \\ \mathrm{C} & -1.882037 & -0.999800 & 2.809038 \\ \mathrm{H} & -2.239549 & -2.014260 & 3.031410 \\ \mathrm{H} & -2.766418 & -0.361861 & 2.737148 \\ \mathrm{H} & -1.302757 & -0.671413 & 3.680468 \\ \mathrm{C} & -3.090886 & 3.189345 & -0.508094 \\ \mathrm{H} & -2.578074 & 2.724519 & -1.360022 \\ \mathrm{H} & -2.573271 & 4.124953 & -0.260871 \\ \mathrm{H} & -4.102439 & 3.444934 & -0.844509 \\ \mathrm{C} & 0.894055 & 2.363218 & 2.823134\end{array}$




$\begin{array}{llll}\mathrm{H} & 0.458374 & 1.400219 & 3.093120 \\ \mathrm{H} & 0.304386 & 3.169650 & 3.270416 \\ \mathrm{H} & 1.928463 & 2.407638 & 3.178120 \\ \mathrm{C} & 4.597801 & -2.465073 & 0.248905 \\ \mathrm{H} & 5.585173 & -2.190932 & 0.612799 \\ \mathrm{C} & 3.740642 & -0.440177 & 1.462496 \\ \mathrm{H} & 2.765436 & 0.009972 & 1.675510 \\ \mathrm{C} & 3.195001 & -3.944898 & -1.013829 \\ \mathrm{H} & 3.083184 & -4.826151 & -1.640768 \\ \mathrm{C} & -3.078485 & -2.825918 & -0.290152 \\ \mathrm{H} & -2.018076 & -2.676049 & -0.079081 \\ \mathrm{C} & 0.712893 & -3.608147 & -1.262118 \\ \mathrm{H} & -0.032454 & -2.906952 & -0.880361 \\ \mathrm{C} & -5.194624 & 0.919639 & 0.142433 \\ \mathrm{H} & -5.745001 & 1.853614 & 0.226736 \\ \mathrm{C} & 4.458078 & -3.596814 & -0.547149 \\ \mathrm{H} & -4.25693 \\ \mathrm{H} & -2.325637 & -4.199606 & -0.803036 \\ \mathrm{C} & -5.174708 & -1.440934 & -0.269157 \\ \mathrm{H} & -5.708391 & -2.355205 & -0.517036 \\ \mathrm{C} & 0.318575 & -5.020287 & -0.787861 \\ \mathrm{H} & 1.020393 & -5.781434 & -1.149788 \\ \mathrm{H} & -0.677248 & -5.287883 & -1.160446 \\ \mathrm{H} & 0.299045 & -5.079202 & 0.306286 \\ \mathrm{C} & 0.688790 & -3.510979 & -2.800393 \\ \mathrm{H} & 0.922105 & -2.493111 & -3.134464 \\ \mathrm{H} & -0.302020 & -3.775641 & -3.188402 \\ \mathrm{H} & 1.419794 & -4.187774 & -3.259422 \\ \mathrm{H} & -3.791434 & 2.940268 & 1.916240 \\ \mathrm{H} & -4.820732 & 3.249426 & 1.702277 \\ \mathrm{H} & -3.229456 & 3.842944 & 2.188120 \\ \mathrm{H} & -3.818556 & 2.278166 & 2.788784 \\ \mathrm{H} & -3.495037 & -3.916299 & 0.670646 \\ \mathrm{H} & -3.605435 & 1.716801 \\ \mathrm{H} & -4.843024 & 0.538699 \\ \mathrm{H} & -4.150902 & 0.491304 \\ \mathrm{H} & -0.248318 & -0.162717 \\ \mathrm{H} & -0.229279 & -0.319174 \\ \mathrm{H} & -3.289934 & -1.755679 \\ \mathrm{H} & -2.462186 & -2.035077 \\ \mathrm{H} & -1.912298\end{array}$




$\begin{array}{llll}\mathrm{H} & -2.792660 & -2.540750 & -2.442589 \\ \mathrm{C} & 4.391305 & -0.793878 & 2.814250 \\ \mathrm{H} & 3.793438 & -1.525078 & 3.367654 \\ \mathrm{H} & 4.496359 & 0.103434 & 3.437832 \\ \mathrm{H} & 5.393207 & -1.217353 & 2.679793 \\ \mathrm{C} & 4.586923 & 0.612943 & 0.720544 \\ \mathrm{H} & 5.585729 & 0.226760 & 0.486409 \\ \mathrm{H} & 4.713726 & 1.516369 & 1.331431 \\ \mathrm{H} & 4.118180 & 0.902960 & -0.226416 \\ \mathrm{Sm} & 0.191586 & 0.753410 & -0.317697 \\ \mathrm{C} & 1.287321 & 3.291715 & -2.033542 \\ \mathrm{C} & 0.452024 & 4.305524 & -1.505666 \\ \mathrm{C} & 2.607475 & 3.241538 & -1.524521 \\ \mathrm{C} & 0.895653 & 5.197748 & -0.525689 \\ \mathrm{H} & -0.564361 & 4.405050 & -1.879887 \\ \mathrm{C} & 3.062715 & 4.129821 & -0.546785 \\ \mathrm{H} & 3.299519 & 2.498489 & -1.917556 \\ \mathrm{C} & 2.202578 & 5.106439 & -0.025883 \\ \mathrm{H} & 0.226387 & 5.971235 & -0.155553 \\ \mathrm{H} & 4.088423 & 4.063915 & -0.191997 \\ \mathrm{H} & 2.555674 & 5.808169 & 0.725409 \\ \mathrm{O} & 0.848975 & 2.445706 & 1.386958 \\ \mathrm{Si} & 0.589416 & 1.869297 & -3.132979 \\ \mathrm{H} & 1.233746 & 3.294048 & 1.082107 \\ \mathrm{H} & -0.435413 & 2.552404 & -3.993654 \\ \mathrm{H} & 1.721314 & 1.554265 & -4.068984\end{array}$

C'

\begin{tabular}{lccc} 
Atom & \multicolumn{1}{c}{$\mathrm{X}$} & $\mathrm{Y}$ & \multicolumn{1}{c}{$\mathrm{Z}$} \\
$\mathrm{Sm}$ & -0.388415 & -0.570604 & 0.254281 \\
$\mathrm{~N}$ & 1.853730 & -0.810612 & 0.206161 \\
$\mathrm{~N}$ & 0.236570 & 1.594799 & 0.559534 \\
$\mathrm{C}$ & 2.883213 & -1.681552 & -0.245938 \\
$\mathrm{C}$ & -0.187497 & 2.941612 & 0.458495 \\
$\mathrm{C}$ & 1.268827 & 1.223375 & 1.467055 \\
$\mathrm{C}$ & -1.534882 & 3.279550 & 0.788158 \\
$\mathrm{C}$ & 1.386596 & 2.015637 & 2.765876 \\
$\mathrm{H}$ & 1.348959 & 1.376232 & 3.656918 \\
$\mathrm{H}$ & 0.573348 & 2.741260 & 2.842647 \\
$\mathrm{H}$ & 2.327188 & 2.578404 & 2.823762
\end{tabular}




\begin{tabular}{|c|c|c|c|}
\hline & 2.893305 & -3.052335 & 0.137778 \\
\hline & 2.057900 & 0.087608 & 1.285965 \\
\hline & 3.880862 & -1.204841 & 1140208 \\
\hline & 1.886868 & -3.636806 & 1.125176 \\
\hline & 1.283488 & -2.805267 & 1506678 \\
\hline & 0.681982 & 3.967282 & -0.011519 \\
\hline & 3.053112 & -0.311187 & 2.362737 \\
\hline & 3.511571 & 0.558421 & 2.840977 \\
\hline & 3.855315 & 0.912197 & 1.926037 \\
\hline & 2.597794 & -0.919920 & 3.159526 \\
\hline & 0.929518 & -4.638550 & 0.450177 \\
\hline & 0.364432 & -4.173523 & -0.36596 \\
\hline & 0.205410 & -5.036026 & 1.172242 \\
\hline & 1.479613 & -5.487930 & 0.028317 \\
\hline & -0.857566 & -1.454029 & 285000 \\
\hline & -0.077531 & -0.705901 & 3.99619 \\
\hline & -0.477825 & -2.438474 & 4.14241 \\
\hline & -1.730117 & -1.192698 & 4.45793 \\
\hline & -1.977801 & 4.596002 & 0.613499 \\
\hline & -3.006204 & 4.848251 & 0.856089 \\
\hline & -2.512393 & 2.235684 & 1.318812 \\
\hline & -1.909606 & 1.426140 & 1.753365 \\
\hline & 0.192284 & 5.270973 & -0.149899 \\
\hline & 0.858702 & 6.048424 & -0.515531 \\
\hline & 3.925215 & 0.252568 & -1.585118 \\
\hline & 3.008478 & 0.727764 & -1.224450 \\
\hline & 2.125140 & 3.690517 & -0.424901 \\
\hline & 2.340219 & 2.640134 & -0.218599 \\
\hline & 3.871239 & -3.904035 & -0.392523 \\
\hline & 3.872183 & -4.953348 & -0.107892 \\
\hline & -1.127096 & 5.594 & 0.149758 \\
\hline $\mathrm{H}$ & -1.488375 & 6.611497 & 0.024610 \\
\hline$C$ & 4.847837 & -2.092422 & -1.625442 \\
\hline $\mathrm{H}$ & 5.612165 & -1.725396 & -2.30588 \\
\hline $\mathrm{C}$ & 3.135413 & 4.535546 & 0.376742 \\
\hline 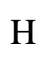 & 3.020107 & 5.606649 & 0.171738 \\
\hline & 4.163051 & 4.257936 & 0.112553 \\
\hline & 3.012161 & 4.390871 & 1.455283 \\
\hline & 2.321307 & 3.916333 & -1.937777 \\
\hline & 1.638735 & 3.292145 & -2.525266 \\
\hline
\end{tabular}




\begin{tabular}{|c|c|c|c|}
\hline $\mathrm{H}$ & 3.347971 & 3.671851 & -2.236697 \\
\hline$U$ & 2.134963 & 4.960754 & -2.215680 \\
\hline$C$ & 2.575240 & -4.281613 & 2.344863 \\
\hline & 3.173633 & -5.154075 & 2.058789 \\
\hline & 1.826427 & -4.623053 & 3.070850 \\
\hline & 3.241649 & -3.572202 & 2.847466 \\
\hline & 5.119250 & 0.994203 & -0.951318 \\
\hline & 5.078825 & 0.949481 & 0.142298 \\
\hline $\mathrm{H}$ & 5.124215 & 2.050476 & -1.247533 \\
\hline & 6.072700 & 0.554104 & -1.268729 \\
\hline $\mathrm{C}$ & 4.847365 & -3.436582 & -1.265429 \\
\hline $\mathrm{H}$ & 5.600911 & -4.112635 & -1.660976 \\
\hline $\mathrm{C}$ & 3.951141 & 0.393643 & -3.119866 \\
\hline $\mathrm{H}$ & 4.871933 & -0.014173 & -3.553182 \\
\hline & 3.894290 & 1.450112 & -3.408385 \\
\hline & 3.108521 & -0.131789 & -3.584289 \\
\hline & -3.418275 & 2.749490 & 2.453009 \\
\hline & -2.829759 & 3.203930 & 3.257560 \\
\hline$\Pi$ & -3.996480 & 1.919548 & 2.876819 \\
\hline $\mathrm{H}$ & -4.139665 & 3.495915 & 2.101792 \\
\hline $\mathrm{C}$ & -3.368569 & 1.639479 & 0.182928 \\
\hline $\mathrm{H}$ & -3.997576 & 2.414710 & -0.271178 \\
\hline $\mathrm{H}$ & -4.025901 & 0.837962 & 0.538802 \\
\hline $\mathrm{H}$ & -2.750733 & 1.230935 & -0.628320 \\
\hline$C$ & -4.517190 & -2.127582 & -0.792843 \\
\hline 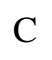 & -5.538509 & -1.720061 & 0.086760 \\
\hline $\mathrm{C}$ & -4.804997 & -2.073810 & -2.171141 \\
\hline$c$ & -6.778681 & -1.278123 & -0.381383 \\
\hline 11 & -5.368741 & -1.762939 & 1.161263 \\
\hline $\mathrm{C}$ & -6.043227 & -1.639158 & -2.647880 \\
\hline $\mathrm{H}$ & -4.051824 & -2.393436 & -2.890862 \\
\hline $\mathrm{C}$ & -7.036655 & -1.235359 & -1.752720 \\
\hline $\mathrm{H}$ & -7.547749 & -0.976190 & 0.326341 \\
\hline $\mathrm{H}$ & -6.234765 & -1.619199 & -3.718540 \\
\hline$H$ & -8.001964 & -0.896201 & -2.120037 \\
\hline$C$ & -1.049165 & 1.621452 & -3.123240 \\
\hline & -2.078469 & 1.286546 & -3.287595 \\
\hline & -1.042744 & 2.381351 & -2.332632 \\
\hline 11 & -0.700468 & 2.118846 & -4.038384 \\
\hline & -0.135493 & 0.479406 & -2.786888 \\
\hline
\end{tabular}




$\begin{array}{llll}\mathrm{H} & 0.901040 & 0.739476 & -2.576043 \\ \mathrm{C} & -0.491436 & -0.818223 & -2.767419 \\ \mathrm{H} & 0.242734 & -1.606075 & -2.600252 \\ \mathrm{H} & -1.503106 & -1.137538 & -3.004464 \\ \mathrm{Si} & -2.777112 & -2.635377 & -0.181597 \\ \mathrm{H} & -2.555461 & -3.990629 & -0.807339 \\ \mathrm{H} & -3.093536 & -3.077946 & 1.263379 \\ \mathrm{O} & -1.195816 & -1.435467 & 2.454656 \\ \mathrm{H} & -1.913492 & -2.073977 & 2.249883\end{array}$

\section{TS4}

Imaginary frequency: $-\mathbf{2 7 5 . 6 9 9 9} \mathrm{cm}^{-1}$

\begin{tabular}{lccc} 
Atom & \multicolumn{1}{c}{$\mathrm{X}$} & \multicolumn{1}{c}{$\mathrm{Y}$} & $\mathrm{Z}$ \\
$\mathrm{Sm}$ & -0.417792 & -0.149538 & 0.214203 \\
$\mathrm{~N}$ & 1.507356 & -1.330220 & 0.285725 \\
$\mathrm{~N}$ & 1.086722 & 1.544635 & 0.398460 \\
$\mathrm{C}$ & 2.049673 & -2.583495 & -0.111779 \\
$\mathrm{C}$ & 1.221127 & 2.931643 & 0.143327 \\
$\mathrm{C}$ & 1.965546 & 0.872564 & 1.289753 \\
$\mathrm{C}$ & 0.195530 & 3.833250 & 0.555281 \\
$\mathrm{C}$ & 2.563138 & 1.680086 & 2.439117 \\
$\mathrm{H}$ & 2.321740 & 1.263210 & 3.425509 \\
$\mathrm{H}$ & 2.187773 & 2.705807 & 2.415250 \\
$\mathrm{H}$ & 3.657543 & 1.737638 & 2.381571 \\
$\mathrm{C}$ & 1.559421 & -3.797555 & 0.446573 \\
$\mathrm{C}$ & 2.181617 & -0.501108 & 1.223754 \\
$\mathrm{C}$ & 3.046684 & -2.643807 & -1.123621 \\
$\mathrm{C}$ & 0.494930 & -3.822480 & 1.539820 \\
$\mathrm{H}$ & 0.301132 & -2.783174 & 1.826632 \\
$\mathrm{C}$ & 2.341257 & 3.450799 & -0.567090 \\
$\mathrm{C}$ & 3.044512 & -1.187293 & 2.267801 \\
$\mathrm{H}$ & 3.778961 & -0.511032 & 2.710509 \\
$\mathrm{H}$ & 3.591533 & -2.018862 & 1.813030 \\
$\mathrm{H}$ & 2.454373 & -1.618056 & 3.092725 \\
$\mathrm{C}$ & -0.830037 & -4.423640 & 1.031219 \\
$\mathrm{H}$ & -1.209267 & -3.880135 & 0.157300 \\
$\mathrm{H}$ & -1.601953 & -4.392271 & 1.810496 \\
$\mathrm{H}$ & -0.700119 & -5.470052 & 0.730989 \\
$\mathrm{C}$ & -0.689531 & -0.381279 & 3.822383
\end{tabular}




\begin{tabular}{|c|c|c|c|}
\hline $\mathrm{H}$ & 0.377275 & -0.348836 & 3.600731 \\
\hline 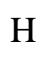 & -0.925721 & -1.301699 & 4.365548 \\
\hline & -0.965353 & 0.493637 & 4.419535 \\
\hline $\mathrm{C}$ & 0.292703 & 5.190684 & 0.223565 \\
\hline $\mathrm{H}$ & -0.498547 & 5.870944 & 0.529888 \\
\hline $\mathrm{C}$ & -1.012214 & 3.377611 & 1.368841 \\
\hline $\mathrm{H}$ & -0.828414 & 2.337786 & 1.664427 \\
\hline C & 2.399951 & 4.818635 & -0.854404 \\
\hline $\mathrm{H}$ & 3.255154 & 5.206081 & -1.403082 \\
\hline $\mathrm{C}$ & 3.590011 & -1.383042 & -1.784384 \\
\hline $\mathrm{H}$ & 3.033630 & -0.538381 & -1.371554 \\
\hline $\mathrm{C}$ & 3.468093 & 2.555098 & -1.071799 \\
\hline & 3.255741 & 1.535707 & -0.744665 \\
\hline $\mathrm{C}$ & 2.063097 & -5.019932 & -0.016327 \\
\hline $\mathrm{H}$ & 1.681761 & -5.946254 & 0.407151 \\
\hline $\mathrm{C}$ & 1.384983 & 5.691973 & -0.474970 \\
\hline $\mathrm{H}$ & 1.445661 & 6.749168 & -0.719810 \\
\hline C & 3.529243 & -3.889537 & -1.540973 \\
\hline $\mathrm{H}$ & 4.292874 & -3.929701 & -2.314223 \\
\hline $\mathrm{C}$ & 4.835914 & 2.954867 & -0.483841 \\
\hline $\mathrm{H}$ & 5.139510 & 3.957473 & -0.809201 \\
\hline $\mathrm{H}$ & 5.613323 & 2.252524 & -0.808479 \\
\hline $\mathrm{H}$ & 4.815650 & 2.954912 & 0.611382 \\
\hline $\mathrm{C}$ & 3.523521 & 2.546948 & -2.612534 \\
\hline $\mathrm{H}$ & 2.569686 & 2.219621 & -3.041123 \\
\hline $\mathrm{H}$ & 4.307720 & 1.867287 & -2.967742 \\
\hline $\mathrm{H}$ & 3.743105 & 3.544110 & -3.012921 \\
\hline $\mathrm{C}$ & 0.971057 & -4.559150 & 2.807143 \\
\hline $\mathrm{H}$ & 1.158104 & -5.621409 & 2.611782 \\
\hline $\mathrm{H}$ & 0.210059 & -4.500327 & 3.595979 \\
\hline $\mathrm{H}$ & 1.899462 & -4.125242 & 3.193958 \\
\hline $\mathrm{C}$ & 5.082259 & -1.170959 & -1.462843 \\
\hline $\mathrm{H}$ & 5.251395 & -1.114251 & -0.381813 \\
\hline $\mathrm{H}$ & 5.447448 & -0.239137 & -1.912033 \\
\hline $\mathrm{H}$ & 5.697037 & -1.990971 & -1.854306 \\
\hline $\mathrm{C}$ & 3.045878 & -5.076370 & -0.998800 \\
\hline $\mathrm{H}$ & 3.428424 & -6.034755 & -1.340450 \\
\hline $\mathrm{C}$ & 3.352775 & -1.391971 & -3.307455 \\
\hline $\mathrm{H}$ & 3.893190 & -2.209801 & -3.799309 \\
\hline $\mathrm{H}$ & 3.695530 & -0.451860 & -3.755608 \\
\hline
\end{tabular}




$\begin{array}{llll}\mathrm{H} & 2.288358 & -1.510212 & -3.541014 \\ \mathrm{C} & -1.181956 & 4.177007 & 2.675870 \\ \mathrm{H} & -0.268505 & 4.143978 & 3.279807 \\ \mathrm{H} & -2.004835 & 3.765775 & 3.274637 \\ \mathrm{H} & -1.414065 & 5.230509 & 2.482635 \\ \mathrm{C} & -2.312372 & 3.414343 & 0.541008 \\ \mathrm{H} & -2.546566 & 4.436966 & 0.222583 \\ \mathrm{H} & -3.164091 & 3.040461 & 1.123915 \\ \mathrm{H} & -2.229336 & 2.807873 & -0.370057 \\ \mathrm{C} & -5.356822 & -0.602450 & -0.492738 \\ \mathrm{C} & -6.063971 & 0.612797 & -0.417904 \\ \mathrm{C} & -5.964354 & -1.670086 & -1.179843 \\ \mathrm{C} & -7.323114 & 0.756598 & -1.001932 \\ \mathrm{H} & -5.629039 & 1.459904 & 0.109479 \\ \mathrm{C} & -7.224037 & -1.531395 & -1.766551 \\ \mathrm{H} & -5.455838 & -2.629684 & -1.248704 \\ \mathrm{C} & -7.906418 & -0.316735 & -1.679384 \\ \mathrm{H} & -7.850122 & 1.704512 & -0.926710 \\ \mathrm{H} & -7.673778 & -2.372828 & -2.287698 \\ \mathrm{H} & -8.887576 & -0.207399 & -2.134026 \\ \mathrm{C} & -0.769925 & 0.876920 & -2.940663 \\ \mathrm{H} & -1.187785 & 1.781620 & -2.475032 \\ \mathrm{H} & 0.319459 & 0.997170 & -2.947343 \\ \mathrm{H} & -1.101588 & 0.890349 & -3.992027 \\ \mathrm{C} & -1.200460 & -0.391034 & -2.213304 \\ \mathrm{H} & -0.667083 & -1.301189 & -2.503817 \\ \mathrm{C} & -2.571086 & -0.565726 & -1.856289 \\ \mathrm{H} & -3.011872 & -1.554027 & -2.015068 \\ \mathrm{H} & -3.262040 & 0.227650 & -2.143830 \\ \mathrm{H} & -3.617357 & -0.748661 & 0.221688 \\ & -3.450045 & -2.167070 & 0.689114 \\ \mathrm{H} & -1.365636 & -0.356863 & 2.551296 \\ \mathrm{H} & -2.335038 & -0.345225 & 2.669151\end{array}$

\section{D}

$\begin{array}{lccc}\text { Atom } & \mathrm{X} & \mathrm{Y} & \mathrm{Z} \\ \mathrm{Sm} & -0.221165 & -0.148788 & 0.244304 \\ \mathrm{~N} & 1.717704 & -1.301945 & 0.339142 \\ \mathrm{~N} & 1.286095 & 1.535544 & 0.480741\end{array}$




\begin{tabular}{|c|c|c|c|}
\hline & 2.230846 & -2.562401 & -0.06952 \\
\hline & 1.402571 & 2.916746 & 0.195695 \\
\hline & 2.197501 & 0.877717 & 1.354546 \\
\hline & 0.356986 & 3.804520 & .590425 \\
\hline & 2.805438 & 1.693530 & 2.490955 \\
\hline & 2.495564 & 1.336838 & 3.482923 \\
\hline & 2.501404 & 2.739860 & 2.409177 \\
\hline & 3.902285 & 1.676238 & 2.477795 \\
\hline & 1.694428 & -3.764756 & 0.472447 \\
\hline & 2.414290 & -0.490436 & 1.277676 \\
\hline & 3.238378 & -2.645693 & -1.068512 \\
\hline & 0.606186 & -3.751202 & 1.542100 \\
\hline & 0.521016 & -2.717445 & 1807426 \\
\hline & 2.513979 & 3.446223 & -0.519352 \\
\hline & 3.280814 & -1.197537 & 2.302189 \\
\hline & 3.980292 & -0.518981 & 2.794779 \\
\hline & 3.866372 & -1.988864 & 1.822965 \\
\hline & 2.685271 & -1.685261 & 3.090467 \\
\hline & -0.761004 & -4.161854 & 0.95820 \\
\hline & -1.050433 & -3.521513 & 0.114565 \\
\hline & -1.550886 & -4.107168 & $1.71942 \&$ \\
\hline & -0.734538 & -5.190609 & 0.580021 \\
\hline & -1.385785 & -0.444551 & 3.73853 \\
\hline & -0.337835 & -0.173766 & $3.87241 \varepsilon$ \\
\hline & -1.564835 & -1.433101 & \\
\hline & -2.020559 & 0.304058 & 4.222229 \\
\hline & 0.423214 & 5.156482 & 0.232469 \\
\hline & -0.382933 & 5.825926 & 0.522119 \\
\hline & -0.840895 & 3.319251 & 1.401077 \\
\hline & -0.561651 & 2.345025 & 1.823783 \\
\hline & 2.542795 & 4.809683 & -0.832360 \\
\hline 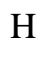 & 3.390521 & 5.205477 & -1.386663 \\
\hline & 3.821851 & -1.396939 & -1.71797 \\
\hline & 3.277670 & -0.540188 & -1.313435 \\
\hline & 3.659214 & 2.564276 & -1.006727 \\
\hline & 3.475248 & 1.549532 & -0.649024 \\
\hline & 2.163760 & -4.999336 & 0.007295 \\
\hline & 1.746033 & -5.916825 & 0.414935 \\
\hline & 1.507025 & 5.667083 & -0.473760 \\
\hline & 1.544629 & 6.720120 & -0.740246 \\
\hline
\end{tabular}




\begin{tabular}{|c|c|c|c|}
\hline C & 3.688731 & -3.903474 & -1.486436 \\
\hline & 4.461387 & -3.962843 & -2.249367 \\
\hline$C$ & 5.020751 & 3.013347 & -0.440092 \\
\hline & 5.297174 & 4.013934 & -0.794382 \\
\hline & 5.812930 & 2.321974 & -0.752626 \\
\hline & 5.008112 & 3.041297 & 0.654997 \\
\hline & 3.702337 & 2.514933 & -2.547097 \\
\hline $\mathrm{H}$ & 2.754540 & 2.150237 & -2.958148 \\
\hline $\mathrm{H}$ & 4.502124 & 1.847084 & -2.889960 \\
\hline 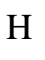 & 3.892335 & 3.506642 & -2.975491 \\
\hline & 0.955979 & -4.618269 & 2.766022 \\
\hline & 1.017123 & -5.682207 & 2.510157 \\
\hline & 0.187681 & -4.514377 & 3.543060 \\
\hline & 1.919230 & -4.323387 & 3.196140 \\
\hline & 5.312697 & -1.216605 & -1.371879 \\
\hline & 5.465630 & -1.161599 & -0.288367 \\
\hline 11 & 5.704532 & -0.293090 & -1.815979 \\
\hline $\mathrm{H}$ & 5.916177 & -2.049914 & -1.752877 \\
\hline C & 3.160115 & -5.078248 & -0.960452 \\
\hline $\mathrm{H}$ & 3.517143 & -6.045553 & -1.304580 \\
\hline $\mathrm{C}$ & 3.609225 & -1.400129 & -3.244801 \\
\hline $\mathrm{H}$ & 4.141990 & -2.227651 & -3.728818 \\
\hline$H$ & 3.976784 & -0.466343 & -3.686217 \\
\hline & 2.546689 & -1.497511 & -3.495834 \\
\hline $\mathrm{C}$ & -1.183056 & 4.231671 & 2.593966 \\
\hline $\mathrm{H}$ & -0.305987 & 4.396563 & 3.228850 \\
\hline 11 & -1.970992 & 3.777738 & 3.208506 \\
\hline $\mathrm{H}$ & -1.550852 & 5.212133 & 2.271050 \\
\hline $\mathrm{C}$ & -2.084550 & 3.113041 & 0.511665 \\
\hline $\mathrm{H}$ & -2.405543 & 4.062317 & 0.066634 \\
\hline $\mathrm{H}$ & -2.928397 & 2.711399 & 1.089404 \\
\hline$H$ & -1.882364 & 2.431142 & -0.325481 \\
\hline $\mathrm{C}$ & -5.927585 & -0.602105 & -0.816850 \\
\hline 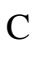 & -6.329738 & 0.746370 & -0.769809 \\
\hline & -6.933172 & -1.585191 & -0.855800 \\
\hline & -7.679622 & 1.098853 & -0.757078 \\
\hline & -5.580802 & 1.535900 & -0.748572 \\
\hline & -8.286002 & -1.238319 & -0.843469 \\
\hline & -6.657920 & -2.636663 & -0.901233 \\
\hline & -8.661064 & 0.105157 & -0.793215 \\
\hline
\end{tabular}




$\begin{array}{llll}\mathrm{H} & -7.966714 & 2.146761 & -0.722771 \\ \mathrm{H} & -9.045317 & -2.015470 & -0.876498 \\ \mathrm{H} & -9.713178 & 0.378048 & -0.785811 \\ \mathrm{C} & -0.747655 & 0.340092 & -3.017931 \\ \mathrm{H} & -0.787519 & 1.429775 & -2.863961 \\ \mathrm{H} & 0.316962 & 0.062495 & -3.048870 \\ \mathrm{H} & -1.146994 & 0.158869 & -4.032604 \\ \mathrm{C} & -1.517457 & -0.421271 & -1.919985 \\ \mathrm{H} & -1.410293 & -1.500464 & -2.151408 \\ \mathrm{C} & -3.024151 & -0.085464 & -2.006284 \\ \mathrm{H} & -3.431688 & -0.232245 & -3.027168 \\ \mathrm{H} & -3.196883 & 0.982841 & -1.792685 \\ \mathrm{Si} & -4.107318 & -1.081270 & -0.833223 \\ \mathrm{H} & -4.020375 & -2.548347 & -1.081144 \\ \mathrm{H} & -3.566093 & -0.903148 & 0.572640 \\ \mathrm{O} & -1.624860 & -0.464629 & 2.317709 \\ \mathrm{H} & -2.550575 & -0.702396 & 2.123796\end{array}$

$\begin{array}{lccc}\text { D' } & & & \\ \text { Atom } & \mathrm{X} & \mathrm{Y} & \mathrm{Z} \\ \mathrm{Sm} & 0.564441 & -1.044280 & 0.283136 \\ \mathrm{~N} & 2.444752 & 0.202082 & 0.114904 \\ \mathrm{~N} & -0.189147 & 0.965349 & 0.994774 \\ \mathrm{C} & 3.709967 & 0.142067 & -0.523968 \\ \mathrm{C} & -1.412070 & 1.677969 & 1.132816 \\ \mathrm{C} & 0.980134 & 1.369007 & 1.698617 \\ \mathrm{C} & -2.382456 & 1.282309 & 2.096923 \\ \mathrm{C} & 0.823682 & 2.153964 & 2.989247 \\ \mathrm{H} & 0.796809 & 1.512843 & 3.883475 \\ \mathrm{H} & -0.107648 & 2.726497 & 2.977386 \\ \mathrm{H} & 1.642786 & 2.864926 & 3.131272 \\ \mathrm{C} & 4.541624 & -1.001886 & -0.362919 \\ \mathrm{C} & 2.250501 & 0.982355 & 1.283977 \\ \mathrm{C} & 4.136209 & 1.193618 & -1.379425 \\ \mathrm{C} & 4.183763 & -2.141538 & 0.588751 \\ \mathrm{H} & 3.285365 & -1.832982 & 1.141658 \\ \mathrm{C} & -1.702215 & 2.761402 & 0.259457 \\ \mathrm{C} & 3.457993 & 1.321157 & 2.148501 \\ \mathrm{H} & 3.670045 & 2.399121 & 2.151946 \\ \mathrm{H} & 4.353588 & 0.822967 & 1.770921\end{array}$




\begin{tabular}{|c|c|c|c|}
\hline & 3.324614 & 1.021577 & 3.195192 \\
\hline & 3.851138 & -3.445835 & -0.162515 \\
\hline & 3.026305 & -3.305798 & -0.87164 \\
\hline & 3.567329 & -4.240702 & 0.538678 \\
\hline & 4.715307 & -3.798755 & -0.7374 \\
\hline & -0.495157 & -4.317509 & $2.05 / 8$ \\
\hline & -0.165705 & -5.321420 & 1.7691 \\
\hline & -1.588612 & 62867 & 0309 \\
\hline & -0.147187 & -4.104762 & 3.0703 \\
\hline & -3.611236 & 1.953970 & 2.146809 \\
\hline & -4.354996 & 1.649457 & 2.879235 \\
\hline & -2.126893 & 0.156028 & 3.096091 \\
\hline & -1.088460 & -0.168326 & 2.96008 \\
\hline & -2.941093 & 3.406157 & 0.354484 \\
\hline & -3.161263 & 4.233571 & -0.31565 \\
\hline & 3.287514 & 2.443240 & -1.587887 \\
\hline & 2.402555 & 2.347444 & -0.954594 \\
\hline & -0.690023 & 3.240500 & -0.77421 \\
\hline & 0.156654 & 2.550586 & -0.735441 \\
\hline & 5.741566 & -1.084887 & -1.08160 \\
\hline & 6.371719 & -1.963591 & -0.96566 \\
\hline & -3.896772 & 3.010392 & 1.286382 \\
\hline & -4.852087 & 3.525787 & 1.347937 \\
\hline & 5.348479 & 1.068906 & -2.066461 \\
\hline & 5.667974 & 1.870516 & -2.72827 \\
\hline & -0.162763 & 4.648344 & -0.4335 \\
\hline & -0.967192 & 5.394252 & -0.45249 \\
\hline & 0.598621 & 4.961726 & 150 \\
\hline & 0.289926 & 4.670424 & 0.563866 \\
\hline & -1.257781 & 3.198327 & -2.2057 \\
\hline & -1.616878 & 194838 & -2.4618 \\
\hline$\Pi$ & -0.484979 & 3.477229 & -2.9322 \\
\hline$\Pi$ & -2.096927 & 3.892878 & -2.3338 \\
\hline $\mathrm{C}$ & 5.280276 & -2.392013 & 1.64269 \\
\hline 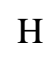 & 6.211617 & -2.739407 & 1.18096 \\
\hline & 4.959042 & -3.161352 & 2.355893 \\
\hline & 5.507070 & -1.479395 & 2.203964 \\
\hline & 4.031278 & 3.722580 & -1.157245 \\
\hline & 4.356366 & 3.661079 & -0.112675 \\
\hline & 3.378986 & 4.598784 & -1.25793 \\
\hline
\end{tabular}




\begin{tabular}{|c|c|c|c|}
\hline$U$ & 4.921738 & 3.900080 & -1.772699 \\
\hline $\mathrm{C}$ & 6.149246 & -0.062149 & -1.931522 \\
\hline 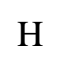 & 7.083430 & -0.143831 & -2.481103 \\
\hline & 2.803681 & 2.557961 & -3.046838 \\
\hline & 3.644427 & 2.667199 & -3.742711 \\
\hline & 2.154055 & 3.433303 & -3.171238 \\
\hline & 2.237116 & 1.669241 & -3.347182 \\
\hline & -2.267774 & 0.626226 & 4.557505 \\
\hline & -1.615872 & 1.480061 & 4.767551 \\
\hline & -2.002242 & -0.184278 & 5.247783 \\
\hline & -3.296772 & 0.927372 & 4.785968 \\
\hline & -3.035430 & -1.061902 & 2.838454 \\
\hline & -4.093868 & -0.800675 & 2.953909 \\
\hline & -2.814462 & -1.869665 & 3.548376 \\
\hline & -2.908703 & -1.455872 & 1.823656 \\
\hline & -4.829930 & -1.419519 & -1.608546 \\
\hline & -4.964269 & -0.186604 & -0.941116 \\
\hline & -5.896757 & -1.846249 & -2.420820 \\
\hline & -6.117524 & 0.586494 & -1.079250 \\
\hline & -4.165938 & 0.185324 & -0.302728 \\
\hline & -7.053647 & -1.076457 & -2.560205 \\
\hline & -5.824067 & -2.791143 & -2.955091 \\
\hline & -7.165365 & 0.142499 & -1.889226 \\
\hline & -6.191378 & 1.535304 & -0.554663 \\
\hline & -7.864685 & -1.426799 & -3.193811 \\
\hline & -8.063788 & 0.744788 & -1.998635 \\
\hline & 0.723909 & -1.936223 & -2.583727 \\
\hline & 1.183072 & -0.959509 & -2.307207 \\
\hline & 1.548468 & -2.659930 & -2.609336 \\
\hline & 0.387269 & -1.781469 & -3.622921 \\
\hline & -0.404601 & -2.383271 & -1.635657 \\
\hline & -0.624179 & -3.436047 & -1.864553 \\
\hline & -1.677732 & -1.529640 & -1.849386 \\
\hline & -1.769055 & -1.174714 & -2.893039 \\
\hline & -1.682681 & -0.581559 & -1.262167 \\
\hline & -3.274845 & -2.464359 & -1.447231 \\
\hline & -3.403244 & -3.627920 & -2.371420 \\
\hline & -3.158127 & -3.027739 & -0.064332 \\
\hline & 0.086902 & -3.333001 & 1.195314 \\
\hline & -0.220048 & -3.439017 & 0.249546 \\
\hline
\end{tabular}


TS5

Imaginary frequency: $-\mathbf{3 6 1 . 2 7 1 5} \mathrm{cm}^{-1}$

\begin{tabular}{lccc} 
Atom & \multicolumn{1}{c}{$\mathrm{X}$} & $\mathrm{Y}$ & $\mathrm{Z}$ \\
$\mathrm{Sm}$ & 0.582860 & -1.028753 & 0.345121 \\
$\mathrm{~N}$ & 2.421865 & 0.247287 & 0.071444 \\
$\mathrm{~N}$ & -0.216225 & 0.981673 & 0.991432 \\
$\mathrm{C}$ & 3.677220 & 0.226952 & -0.593628 \\
$\mathrm{C}$ & -1.456363 & 1.652333 & 1.180240 \\
$\mathrm{C}$ & 0.957214 & 1.392754 & 1.679265 \\
$\mathrm{C}$ & -2.386543 & 1.209836 & 2.162987 \\
$\mathrm{C}$ & 0.817620 & 2.167346 & 2.978927 \\
$\mathrm{H}$ & 0.907587 & 1.530490 & 3.871323 \\
$\mathrm{H}$ & -0.157646 & 2.657310 & 3.028515 \\
$\mathrm{H}$ & 1.581244 & 2.946520 & 3.067023 \\
$\mathrm{C}$ & 4.585698 & -0.850476 & -0.397419 \\
$\mathrm{C}$ & 2.229399 & 1.026049 & 1.242454 \\
$\mathrm{C}$ & 4.012757 & 1.261427 & -1.507933 \\
$\mathrm{C}$ & 4.319246 & -1.975218 & 0.600614 \\
$\mathrm{H}$ & 3.393412 & -1.725427 & 1.136214 \\
$\mathrm{C}$ & -1.802063 & 2.741864 & 0.335775 \\
$\mathrm{C}$ & 3.439319 & 1.392566 & 2.091175 \\
$\mathrm{H}$ & 3.586145 & 2.479661 & 2.142065 \\
$\mathrm{H}$ & 4.350564 & 0.967103 & 1.666563 \\
$\mathrm{H}$ & 3.353659 & 1.037044 & 3.125906 \\
$\mathrm{C}$ & 4.105213 & -3.332231 & -0.097918 \\
$\mathrm{H}$ & 3.280878 & -3.288722 & -0.818909 \\
$\mathrm{H}$ & 3.876371 & -4.116669 & 0.634041 \\
$\mathrm{H}$ & 5.003041 & -3.638220 & -0.647612 \\
$\mathrm{C}$ & -0.136537 & -4.528091 & 1.709443 \\
$\mathrm{H}$ & 0.407878 & -5.355950 & 1.237316 \\
$\mathrm{H}$ & -1.214469 & -4.720662 & 1.638113 \\
$\mathrm{H}$ & 0.142014 & -4.485606 & 2.766669 \\
$\mathrm{C}$ & -3.633710 & 1.842157 & 2.256795 \\
$\mathrm{H}$ & -4.347444 & 1.502481 & 3.003294 \\
$\mathrm{H}$ & -2.071590 & 0.078188 & 3.139668 \\
$\mathrm{H}$ & -3.3200086 & 4.177960 & -0.173985
\end{tabular}




\begin{tabular}{|c|c|c|c|}
\hline C & 3.077640 & 2.440309 & -1.752662 \\
\hline $\mathrm{H}$ & 2.183699 & 2.275331 & -1.146579 \\
\hline $\mathrm{C}$ & -0.832440 & 3.270900 & -0.713976 \\
\hline $\mathrm{H}$ & 0.044799 & 2.619750 & -0.694786 \\
\hline $\mathrm{C}$ & 5.779180 & -0.880564 & -1.131189 \\
\hline $\mathrm{H}$ & 6.471122 & -1.707020 & -0.987130 \\
\hline $\mathrm{C}$ & -3.975168 & 2.903241 & 1.423050 \\
\hline $\mathrm{H}$ & -4.944169 & 3.386752 & 1.519032 \\
\hline $\mathrm{C}$ & 5.221398 & 1.190928 & -2.208936 \\
\hline $\mathrm{H}$ & 5.473657 & 1.980977 & -2.912354 \\
\hline $\mathrm{C}$ & -0.362229 & 4.699593 & -0.377112 \\
\hline $\mathrm{H}$ & -1.198357 & 5.409928 & -0.379137 \\
\hline $\mathrm{H}$ & 0.371941 & 5.048330 & -1.113965 \\
\hline $\mathrm{H}$ & 0.106523 & 4.738730 & 0.612359 \\
\hline C & -1.426467 & 3.207922 & -2.134038 \\
\hline $\mathrm{H}$ & -1.745723 & 2.190306 & -2.386968 \\
\hline $\mathrm{H}$ & -0.682458 & 3.524141 & -2.875256 \\
\hline $\mathrm{H}$ & -2.298643 & 3.864259 & -2.240919 \\
\hline C & 5.427305 & -2.089155 & 1.666565 \\
\hline $\mathrm{H}$ & 6.387163 & -2.372963 & 1.219789 \\
\hline $\mathrm{H}$ & 5.168678 & -2.855754 & 2.407433 \\
\hline $\mathrm{H}$ & 5.572929 & -1.140701 & 2.194289 \\
\hline $\mathrm{C}$ & 3.709872 & 3.771731 & -1.302484 \\
\hline $\mathrm{H}$ & 3.997682 & 3.737624 & -0.245910 \\
\hline $\mathrm{H}$ & 3.001882 & 4.599342 & -1.433560 \\
\hline $\mathrm{H}$ & 4.609312 & 4.008078 & -1.884533 \\
\hline $\mathrm{C}$ & 6.103021 & 0.128097 & -2.032540 \\
\hline $\mathrm{H}$ & 7.034532 & 0.087155 & -2.591134 \\
\hline $\mathrm{C}$ & 2.629559 & 2.517220 & -3.225152 \\
\hline $\mathrm{H}$ & 3.476846 & 2.693407 & -3.898830 \\
\hline $\mathrm{H}$ & 1.916868 & 3.338831 & -3.368581 \\
\hline $\mathrm{H}$ & 2.142764 & 1.587183 & -3.540394 \\
\hline $\mathrm{C}$ & -2.172708 & 0.531346 & 4.610139 \\
\hline $\mathrm{H}$ & -1.528877 & 1.393867 & 4.809501 \\
\hline $\mathrm{H}$ & -1.872119 & -0.282045 & 5.282085 \\
\hline $\mathrm{H}$ & -3.198932 & 0.812682 & 4.873292 \\
\hline $\mathrm{C}$ & -2.961028 & -1.157543 & 2.901789 \\
\hline П & -4.019990 & -0.919644 & 3.056477 \\
\hline $\mathrm{H}$ & -2.696749 & -1.966010 & 3.594996 \\
\hline $\mathrm{H}$ & -2.859985 & -1.542370 & 1.881035 \\
\hline
\end{tabular}




$\begin{array}{llll}\mathrm{C} & -4.798063 & -1.476063 & -1.611492 \\ \mathrm{C} & -4.932631 & -0.248140 & -0.934718 \\ \mathrm{C} & -5.820121 & -1.849942 & -2.503495 \\ \mathrm{C} & -6.042367 & 0.571770 & -1.141977 \\ \mathrm{H} & -4.169809 & 0.082287 & -0.232937 \\ \mathrm{C} & -6.933837 & -1.033431 & -2.711705 \\ \mathrm{H} & -5.746618 & -2.790448 & -3.045278 \\ \mathrm{C} & -7.045970 & 0.180076 & -2.031104 \\ \mathrm{H} & -6.117230 & 1.515400 & -0.608400 \\ \mathrm{H} & -7.711081 & -1.343634 & -3.405668 \\ \mathrm{H} & -7.910772 & 0.818473 & -2.193621 \\ \mathrm{C} & 0.747410 & -2.182739 & -2.424976 \\ \mathrm{H} & 1.184902 & -1.183430 & -2.201503 \\ \mathrm{H} & 1.579916 & -2.896067 & -2.383282 \\ \mathrm{H} & 0.436179 & -2.098212 & -3.478544 \\ \mathrm{C} & -0.409506 & -2.592892 & -1.489957 \\ \mathrm{H} & -0.661614 & -3.631078 & -1.750380 \\ \mathrm{C} & -1.656828 & -1.703750 & -1.729054 \\ \mathrm{H} & -1.712924 & -1.357355 & -2.776558 \\ \mathrm{H} & -1.653218 & -0.751340 & -1.149512 \\ \mathrm{Si} & -3.298526 & -2.581661 & -1.358802 \\ \mathrm{H} & -3.425257 & -3.752361 & -2.273786 \\ \mathrm{H} & -3.256242 & -3.113834 & 0.036142 \\ \mathrm{O} & 0.200140 & -3.289715 & 1.101434 \\ \mathrm{H} & -0.115783 & -3.212066 & 0.043203\end{array}$

6

$\begin{array}{llll}\text { Atom } & \mathrm{X} & \mathrm{Y} & \mathrm{Z}\end{array}$

$\begin{array}{llll}\text { Sm } & -0.077850 & 1.060635 & 0.040223\end{array}$

$\begin{array}{llll}\mathrm{N} & -1.211499 & -0.823942 & 0.518628\end{array}$

N $\quad 1.634835 \quad-0.306045 \quad 0.481896$

$\begin{array}{llll}\text { C } & -2.513097 & -1.367149 & 0.332889\end{array}$

$\begin{array}{llll}\text { C } & 3.015413 & -0.489324 & 0.208085\end{array}$

$\begin{array}{llll}\text { C } & 1.008667 & -1.014401 & 1.548310\end{array}$

$\begin{array}{llll}\text { C } & 3.947501 & 0.547889 & 0.493007\end{array}$

$\begin{array}{llll}\text { C } & 1.851028 & -1.381404 & 2.762962\end{array}$

$\begin{array}{llll}\mathrm{H} & 1.461844 & -0.947940 & 3.693539\end{array}$

$\begin{array}{llll}\mathrm{H} & 2.877714 & -1.031322 & 2.635896\end{array}$

$\begin{array}{llll}\mathrm{H} & 1.899818 & -2.467409 & 2.917319\end{array}$

$\begin{array}{llll}\text { C } & -3.646822 & -0.740607 & 0.921673\end{array}$ 


\begin{tabular}{|c|c|c|c|}
\hline $\mathrm{C}$ & -0.357533 & -1.277554 & 1.556794 \\
\hline $\mathrm{C}$ & -2.699982 & -2.502981 & -0.499471 \\
\hline $\mathrm{C}$ & -3.522908 & 0.467104 & 1.848323 \\
\hline $\mathrm{H}$ & -2.450872 & 0.653757 & 1.999751 \\
\hline $\mathrm{C}$ & 3.479878 & -1.691569 & -0.392470 \\
\hline $\mathrm{C}$ & -0.974580 & -1.953657 & 2.768923 \\
\hline $\mathrm{H}$ & -0.332239 & -2.749803 & 3.156852 \\
\hline $\mathrm{H}$ & -1.935628 & -2.402930 & 2.506820 \\
\hline $\mathrm{H}$ & -1.157856 & -1.256087 & 3.599877 \\
\hline C & -4.139184 & 1.735522 & 1.225938 \\
\hline $\mathrm{H}$ & -3.689021 & 1.973266 & 0.255065 \\
\hline $\mathrm{H}$ & -4.003393 & 2.601929 & 1.885841 \\
\hline $\mathrm{H}$ & -5.215819 & 1.609149 & 1.062377 \\
\hline $\mathrm{C}$ & 5.299511 & 0.361496 & 0.175495 \\
\hline $\mathrm{H}$ & 6.011683 & 1.153100 & 0.396758 \\
\hline C & 3.543946 & 1.861528 & 1.158459 \\
\hline $\mathrm{H}$ & 2.456154 & 1.827049 & 1.316626 \\
\hline $\mathrm{C}$ & 4.840658 & -1.830388 & -0.684745 \\
\hline $\mathrm{H}$ & 5.191145 & -2.748915 & -1.149379 \\
\hline C & -1.521658 & -3.219019 & -1.148510 \\
\hline $\mathrm{H}$ & -0.624864 & -2.641951 & -0.911460 \\
\hline $\mathrm{C}$ & 2.526710 & -2.822875 & -0.762311 \\
\hline $\mathrm{H}$ & 1.525659 & -2.529293 & -0.440465 \\
\hline $\mathrm{C}$ & -4.924978 & -1.246816 & 0.653996 \\
\hline $\mathrm{H}$ & -5.792743 & -0.765334 & 1.098665 \\
\hline $\mathrm{C}$ & 5.753342 & -0.816676 & -0.406769 \\
\hline $\mathrm{H}$ & 6.806337 & -0.942466 & -0.644713 \\
\hline $\mathrm{C}$ & -3.997236 & -2.976532 & -0.728618 \\
\hline $\mathrm{H}$ & -4.139174 & -3.845502 & -1.366654 \\
\hline $\mathrm{C}$ & 2.880106 & -4.137045 & -0.039215 \\
\hline $\mathrm{H}$ & 3.864040 & -4.515257 & -0.342697 \\
\hline $\mathrm{H}$ & 2.141338 & -4.913739 & -0.271483 \\
\hline $\mathrm{H}$ & 2.897495 & -4.001045 & 1.047811 \\
\hline $\mathrm{C}$ & 2.477369 & -3.033282 & -2.288639 \\
\hline $\mathrm{H}$ & 2.176360 & -2.114287 & -2.804167 \\
\hline $\mathrm{H}$ & 1.756698 & -3.819121 & -2.546396 \\
\hline $\mathrm{H}$ & 3.453974 & -3.334547 & -2.686691 \\
\hline $\mathrm{C}$ & -4.129240 & 0.202459 & 3.240527 \\
\hline $\mathrm{H}$ & -5.212119 & 0.042814 & 3.181581 \\
\hline $\mathrm{H}$ & -3.958261 & 1.059482 & 3.903830 \\
\hline
\end{tabular}




$\begin{array}{llll}\mathrm{H} & -3.687089 & -0.684052 & 3.706325 \\ \mathrm{C} & -1.336663 & -4.635587 & -0.570118 \\ \mathrm{H} & -1.200589 & -4.605471 & 0.516582 \\ \mathrm{H} & -0.455788 & -5.120681 & -1.008871 \\ \mathrm{H} & -2.205714 & -5.271303 & -0.780482 \\ \mathrm{C} & -5.108083 & -2.357571 & -0.163545 \\ \mathrm{H} & -6.107777 & -2.737592 & -0.357553 \\ \mathrm{C} & -1.648025 & -3.264038 & -2.683494 \\ \mathrm{H} & -2.520244 & -3.846021 & -3.005201 \\ \mathrm{H} & -0.759442 & -3.728998 & -3.127308 \\ \mathrm{H} & -1.746526 & -2.256129 & -3.101683 \\ \mathrm{C} & 4.186567 & 2.029975 & 2.549467 \\ \mathrm{H} & 3.928582 & 1.195341 & 3.209872 \\ \mathrm{H} & 3.847947 & 2.959578 & 3.024100 \\ \mathrm{H} & 5.279537 & 2.072199 & 2.477885 \\ \mathrm{C} & 3.851526 & 3.081310 & 0.268490 \\ \mathrm{H} & 4.930574 & 3.200403 & 0.118188 \\ \mathrm{H} & 3.480088 & 4.006041 & 0.728444 \\ \mathrm{H} & 3.397879 & 2.980235 & -0.724385 \\ \mathrm{C} & -0.004999 & 1.693877 & -2.458318 \\ \mathrm{H} & 1.076683 & 1.805848 & -2.606419 \\ \mathrm{C} & -0.672394 & 0.853261 & -3.534196 \\ \mathrm{H} & -1.665717 & 0.490930 & -3.235064 \\ \mathrm{H} & -0.068607 & -0.035482 & -3.746901 \\ \mathrm{H} & -0.806528 & 1.391681 & -4.487314 \\ \mathrm{C} & -0.634199 & 2.900317 & -1.965468 \\ \mathrm{C} & 0.075898 & 3.771092 & -1.074823 \\ \mathrm{C} & -2.027086 & 3.181385 & -2.113068 \\ \mathrm{C} & -0.551540 & 4.831880 & -0.408543 \\ \mathrm{H} & 1.160053 & 3.668057 & -0.997370 \\ \mathrm{H} & -2.636861 & 4.237150 & -1.444461 \\ & -2.617643 & 2.569334 & -2.788133 \\ \mathrm{H} & -1.915538 & 5.069931 & -0.574397 \\ \mathrm{H} & -3.6966511 & 4.420873 & -1.606294 \\ \mathrm{H} & 5.891307 & -0.060043\end{array}$

\section{TS6}

Imaginary frequency: $-104.73 \mathrm{~cm}^{-1}$ 


\begin{tabular}{|c|c|c|c|}
\hline Atom & $\mathrm{X}$ & $\mathrm{Y}$ & $\mathrm{Z}$ \\
\hline $\mathrm{Sm}$ & -0.339287 & 0.076452 & 0.412906 \\
\hline $\mathrm{N}$ & 1.128695 & 1.483546 & -0.532930 \\
\hline $\mathrm{N}$ & 0.912161 & -1.408784 & -0.726769 \\
\hline $\mathrm{C}$ & 1.661969 & 2.799363 & -0.388114 \\
\hline $\mathrm{C}$ & 1.287522 & -2.779369 & -0.797302 \\
\hline $\mathrm{C}$ & 1.002348 & -0.577744 & -1.874299 \\
\hline $\mathrm{C}$ & 0.298934 & -3.803269 & -0.801261 \\
\hline $\mathrm{C}$ & 0.838375 & -1.216795 & -3.247700 \\
\hline $\mathrm{H}$ & 0.631365 & -2.284226 & -3.148831 \\
\hline $\mathrm{H}$ & 1.748477 & -1.119627 & -3.854007 \\
\hline $\mathrm{H}$ & 0.021398 & -0.767739 & -3.825771 \\
\hline $\mathrm{C}$ & 0.839433 & 3.952844 & -0.530556 \\
\hline $\mathrm{C}$ & 1.129844 & 0.810817 & -1.782792 \\
\hline $\mathrm{C}$ & 3.027104 & 2.962890 & -0.028092 \\
\hline $\mathrm{C}$ & -0.635120 & 3.881567 & -0.920196 \\
\hline $\mathrm{H}$ & -0.887101 & 2.828909 & -1.087670 \\
\hline $\mathrm{C}$ & 2.661319 & -3.145630 & -0.818356 \\
\hline $\mathrm{C}$ & 1.139821 & 1.635920 & -3.057888 \\
\hline $\mathrm{H}$ & 1.585081 & 2.615711 & -2.869804 \\
\hline $\mathrm{H}$ & 0.132927 & 1.807703 & -3.465164 \\
\hline $\mathrm{H}$ & 1.726153 & 1.152486 & -3.844721 \\
\hline $\mathrm{C}$ & -1.550054 & 4.400649 & 0.206611 \\
\hline $\mathrm{H}$ & -1.370810 & 3.870413 & 1.148734 \\
\hline $\mathrm{H}$ & -2.605587 & 4.270959 & -0.061867 \\
\hline $\mathrm{H}$ & -1.382109 & 5.467446 & 0.395464 \\
\hline $\mathrm{C}$ & 0.703147 & -5.145271 & -0.803493 \\
\hline $\mathrm{H}$ & -0.053898 & -5.925879 & -0.801544 \\
\hline $\mathrm{C}$ & -1.198310 & -3.510133 & -0.821821 \\
\hline $\mathrm{H}$ & -1.322243 & -2.422723 & -0.895799 \\
\hline $\mathrm{C}$ & 3.011934 & -4.499750 & -0.832646 \\
\hline $\mathrm{H}$ & 4.064008 & -4.774865 & -0.846018 \\
\hline $\mathrm{C}$ & 3.957193 & 1.769591 & 0.152054 \\
\hline $\mathrm{H}$ & 3.370194 & 0.869656 & -0.042186 \\
\hline $\mathrm{C}$ & 3.771185 & -2.101215 & -0.802667 \\
\hline $\mathrm{H}$ & 3.295893 & -1.119233 & -0.764914 \\
\hline $\mathrm{C}$ & 1.396277 & 5.219043 & -0.306235 \\
\hline $\mathrm{H}$ & 0.767281 & 6.099311 & -0.413421 \\
\hline $\mathrm{C}$ & 2.046473 & -5.501948 & -0.820380 \\
\hline $\mathrm{H}$ & 2.338329 & -6.548978 & -0.825359 \\
\hline
\end{tabular}




\begin{tabular}{|c|c|c|c|}
\hline $\mathrm{C}$ & 3.534718 & 4.250346 & 0.181619 \\
\hline $\mathrm{H}$ & 4.579070 & 4.369557 & 0.459985 \\
\hline C & 4.628939 & -2.157179 & -2.081947 \\
\hline $\mathrm{H}$ & 5.160580 & -3.112648 & -2.168540 \\
\hline $\mathrm{H}$ & 5.379474 & -1.357498 & -2.079128 \\
\hline $\mathrm{H}$ & 4.011542 & -2.039078 & -2.979159 \\
\hline $\mathrm{C}$ & 4.654432 & -2.236033 & 0.453388 \\
\hline $\mathrm{H}$ & 4.055034 & -2.157591 & 1.367805 \\
\hline $\mathrm{H}$ & 5.416720 & -1.447577 & 0.476218 \\
\hline $\mathrm{H}$ & 5.175921 & -3.200331 & 0.480467 \\
\hline $\mathrm{C}$ & -0.931556 & 4.636258 & -2.231861 \\
\hline $\mathrm{H}$ & -0.745015 & 5.711469 & -2.126792 \\
\hline $\mathrm{H}$ & -1.983720 & 4.510164 & -2.515287 \\
\hline $\mathrm{H}$ & -0.310668 & 4.270562 & -3.055697 \\
\hline $\mathrm{C}$ & 5.122551 & 1.797633 & -0.856121 \\
\hline $\mathrm{H}$ & 4.752448 & 1.816228 & -1.887113 \\
\hline $\mathrm{H}$ & 5.756660 & 0.910271 & -0.739103 \\
\hline $\mathrm{H}$ & 5.758171 & 2.679991 & -0.713698 \\
\hline $\mathrm{C}$ & 2.731991 & 5.378036 & 0.045852 \\
\hline $\mathrm{H}$ & 3.142234 & 6.370428 & 0.213958 \\
\hline $\mathrm{C}$ & 4.485408 & 1.676203 & 1.597256 \\
\hline $\mathrm{H}$ & 5.101990 & 2.544061 & 1.860359 \\
\hline $\mathrm{H}$ & 5.102008 & 0.778537 & 1.726246 \\
\hline $\mathrm{H}$ & 3.661096 & 1.629181 & 2.319484 \\
\hline $\mathrm{C}$ & -1.897872 & -4.116192 & -2.054567 \\
\hline $\mathrm{H}$ & -1.435967 & -3.769355 & -2.985395 \\
\hline $\mathrm{H}$ & -2.956444 & -3.830195 & -2.072280 \\
\hline $\mathrm{H}$ & -1.849706 & -5.211353 & -2.047750 \\
\hline $\mathrm{C}$ & -1.889544 & -3.971067 & 0.475560 \\
\hline $\mathrm{H}$ & -1.811890 & -5.058036 & 0.597222 \\
\hline $\mathrm{H}$ & -2.954468 & -3.709579 & 0.463403 \\
\hline $\mathrm{H}$ & -1.435265 & -3.508482 & 1.359784 \\
\hline $\mathrm{C}$ & -4.700810 & 0.262039 & -0.443465 \\
\hline $\mathrm{C}$ & -4.421990 & 0.597904 & -1.779175 \\
\hline $\mathrm{C}$ & -6.017271 & -0.120284 & -0.132419 \\
\hline $\mathrm{C}$ & -5.403531 & 0.532726 & -2.769891 \\
\hline $\mathrm{H}$ & -3.418583 & 0.925770 & -2.051532 \\
\hline $\mathrm{C}$ & -7.008488 & -0.187922 & -1.114151 \\
\hline $\mathrm{H}$ & -6.273712 & -0.366913 & 0.897043 \\
\hline $\mathrm{C}$ & -6.701510 & 0.137135 & -2.437172 \\
\hline
\end{tabular}




$\begin{array}{llll}\mathrm{H} & -5.161221 & 0.797388 & -3.796420 \\ \mathrm{H} & -8.019253 & -0.487981 & -0.847999 \\ \mathrm{H} & -7.471191 & 0.090976 & -3.203555 \\ \mathrm{H} & -2.413475 & 1.154535 & 0.037235 \\ \mathrm{C} & -1.852348 & 1.013523 & 2.643536 \\ \mathrm{H} & -1.256265 & 1.861629 & 2.259988 \\ \mathrm{Si} & -3.358641 & 0.265649 & 0.923742 \\ \mathrm{H} & -4.293981 & 0.197206 & 2.093230 \\ \mathrm{H} & -2.644492 & -1.060968 & 0.777394 \\ \mathrm{C} & -0.942969 & 0.003016 & 3.234220 \\ \mathrm{C} & -1.430192 & -1.178248 & 3.853038 \\ \mathrm{C} & 0.469906 & 0.113245 & 3.105631 \\ \mathrm{C} & -0.566895 & -2.168750 & 4.302923 \\ \mathrm{H} & -2.501635 & -1.305535 & 3.976391 \\ \mathrm{C} & 1.337284 & -0.901085 & 3.552647 \\ \mathrm{H} & 0.901900 & 1.058880 & 2.767004 \\ \mathrm{C} & 0.824184 & -2.046070 & 4.147961 \\ \mathrm{H} & -0.977622 & -3.056757 & 4.776695 \\ \mathrm{H} & 2.410155 & -0.768071 & 3.443910 \\ \mathrm{H} & 1.487057 & -2.831483 & 4.497359 \\ \mathrm{C} & -2.878014 & 1.593217 & 3.626213 \\ \mathrm{H} & -2.381316 & 2.074206 & 4.480338 \\ \mathrm{H} & -3.512006 & 2.343681 & 3.139828 \\ \mathrm{H} & -3.547647 & 0.822846 & 4.022668\end{array}$

TS7

Imaginary frequency: $-\mathbf{2 1 9 . 3 1} \mathrm{cm}^{-1}$

$\begin{array}{lccc}\text { Atom } & \mathrm{X} & \mathrm{Y} & \mathrm{Z} \\ \mathrm{Sm} & 0.470101 & -0.328246 & 0.180840 \\ \mathrm{~N} & -1.463304 & -1.282021 & -0.485312 \\ \mathrm{~N} & -0.612446 & 1.509479 & -0.632539 \\ \mathrm{C} & -2.370607 & -2.367074 & -0.328850 \\ \mathrm{C} & -0.628615 & 2.934139 & -0.579007 \\ \mathrm{C} & -1.085973 & 0.809641 & -1.763754 \\ \mathrm{C} & 0.502876 & 3.701441 & -0.976063 \\ \mathrm{C} & -1.070273 & 1.499669 & -3.119253 \\ \mathrm{H} & -1.104507 & 2.583960 & -2.990207 \\ \mathrm{H} & -1.931499 & 1.210689 & -3.728814 \\ \mathrm{H} & -0.168671 & 1.270204 & -3.705979\end{array}$




\begin{tabular}{|c|c|c|c|}
\hline & -1.896633 & -3.700434 & -0.153250 \\
\hline $\mathrm{C}$ & -1.460857 & -0.537307 & -1.701294 \\
\hline $\mathrm{C}$ & -3.777861 & -2.141202 & -0.314651 \\
\hline $\mathrm{C}$ & -0.416821 & -4.070631 & -0.150309 \\
\hline $\mathrm{H}$ & 0.157707 & -3.138882 & -0.170211 \\
\hline $\mathrm{C}$ & -1.770340 & 3.610245 & -0.069260 \\
\hline $\mathrm{C}$ & -1.771659 & -1.282469 & -2.994919 \\
\hline $\mathrm{H}$ & -2.724937 & -0.963475 & -3.436599 \\
\hline $\mathrm{H}$ & -1.851043 & -2.355419 & -2.805610 \\
\hline $\mathrm{H}$ & -1.002332 & -1.131597 & -3.762010 \\
\hline $\mathrm{C}$ & -0.000606 & -4.830836 & 1.123617 \\
\hline $\mathrm{H}$ & -0.287449 & -4.282939 & 2.028077 \\
\hline $\mathrm{H}$ & 1.085469 & -4.986291 & 1.144986 \\
\hline $\mathrm{H}$ & -0.472443 & -5.818544 & 1.176765 \\
\hline $\mathrm{C}$ & 0.470259 & 5.095924 & -0.843406 \\
\hline $\mathrm{H}$ & 1.337130 & 5.677871 & -1.146974 \\
\hline $\mathrm{C}$ & 1.768991 & 3.075397 & -1.553021 \\
\hline $\mathrm{H}$ & 1.604028 & 1.992420 & -1.613464 \\
\hline $\mathrm{C}$ & -1.754981 & 5.005680 & 0.039378 \\
\hline $\mathrm{H}$ & -2.632009 & 5.514952 & 0.431210 \\
\hline $\mathrm{C}$ & -4.385622 & -0.743370 & -0.377909 \\
\hline $\mathrm{H}$ & -3.577085 & -0.028811 & -0.536640 \\
\hline $\mathrm{C}$ & -3.026045 & 2.860391 & 0.356030 \\
\hline $\mathrm{H}$ & -2.808317 & 1.793062 & 0.274567 \\
\hline $\mathrm{C}$ & -2.815498 & -4.748104 & -0.009203 \\
\hline $\mathrm{H}$ & -2.443599 & -5.762826 & 0.109157 \\
\hline $\mathrm{C}$ & -0.646020 & 5.753967 & -0.340274 \\
\hline $\mathrm{H}$ & -0.651998 & 6.836944 & -0.247378 \\
\hline $\mathrm{C}$ & -4.651173 & -3.225139 & -0.174320 \\
\hline $\mathrm{H}$ & -5.722356 & -3.040025 & -0.166526 \\
\hline $\mathrm{C}$ & -4.204249 & 3.181387 & -0.585275 \\
\hline $\mathrm{H}$ & -4.460600 & 4.247616 & -0.555085 \\
\hline $\mathrm{H}$ & -5.099003 & 2.617150 & -0.296631 \\
\hline $\mathrm{H}$ & -3.962808 & 2.926983 & -1.623411 \\
\hline $\mathrm{C}$ & -3.400486 & 3.144812 & 1.823343 \\
\hline $\mathrm{H}$ & -2.571848 & 2.896514 & 2.495411 \\
\hline $\mathrm{H}$ & -4.269882 & 2.545847 & 2.119483 \\
\hline $\mathrm{H}$ & -3.657170 & 4.199350 & 1.981968 \\
\hline $\mathrm{C}$ & -0.029380 & -4.869553 & -1.410521 \\
\hline $\mathrm{H}$ & -0.582007 & -5.814874 & -1.460610 \\
\hline
\end{tabular}




\begin{tabular}{|c|c|c|c|}
\hline $\mathrm{H}$ & 1.041517 & -5.109952 & -1.411395 \\
\hline $\mathrm{H}$ & -0.254333 & -4.307101 & -2.323479 \\
\hline $\mathrm{C}$ & -5.381066 & -0.584425 & -1.544115 \\
\hline $\mathrm{H}$ & -4.918651 & -0.842619 & -2.503041 \\
\hline $\mathrm{H}$ & -5.735734 & 0.451148 & -1.606757 \\
\hline $\mathrm{H}$ & -6.261133 & -1.226459 & -1.418186 \\
\hline $\mathrm{C}$ & -4.186817 & -4.527437 & -0.027454 \\
\hline $\mathrm{H}$ & -4.882287 & -5.355488 & 0.080990 \\
\hline $\mathrm{C}$ & -5.060453 & -0.391435 & 0.964029 \\
\hline $\mathrm{H}$ & -5.882981 & -1.080461 & 1.190385 \\
\hline $\mathrm{H}$ & -5.474664 & 0.623425 & 0.937344 \\
\hline $\mathrm{H}$ & -4.343816 & -0.445002 & 1.791128 \\
\hline $\mathrm{C}$ & 2.070666 & 3.567104 & -2.983066 \\
\hline $\mathrm{H}$ & 1.225403 & 3.388258 & -3.654778 \\
\hline $\mathrm{H}$ & 2.947461 & 3.048828 & -3.390911 \\
\hline $\mathrm{H}$ & 2.285914 & 4.641837 & -2.998339 \\
\hline $\mathrm{C}$ & 2.988101 & 3.315922 & -0.641839 \\
\hline $\mathrm{H}$ & 3.202950 & 4.386028 & -0.542683 \\
\hline $\mathrm{H}$ & 3.883807 & 2.835551 & -1.053464 \\
\hline $\mathrm{H}$ & 2.816181 & 2.924962 & 0.368203 \\
\hline $\mathrm{C}$ & 4.562513 & -0.993900 & -0.522529 \\
\hline $\mathrm{C}$ & 5.289122 & 0.137088 & -0.094688 \\
\hline $\mathrm{C}$ & 5.297866 & -2.162304 & -0.803863 \\
\hline $\mathrm{C}$ & 6.675523 & 0.108157 & 0.032534 \\
\hline $\mathrm{H}$ & 4.753007 & 1.055546 & 0.132290 \\
\hline $\mathrm{C}$ & 6.687495 & -2.192207 & -0.674891 \\
\hline $\mathrm{H}$ & 4.784716 & -3.060035 & -1.135360 \\
\hline $\mathrm{C}$ & 7.386839 & -1.059338 & -0.257895 \\
\hline $\mathrm{H}$ & 7.203089 & 1.001829 & 0.356447 \\
\hline $\mathrm{H}$ & 7.224726 & -3.108830 & -0.905569 \\
\hline $\mathrm{H}$ & 8.468641 & -1.083696 & -0.161498 \\
\hline $\mathrm{C}$ & 1.613178 & -1.439461 & 2.272590 \\
\hline $\mathrm{H}$ & 1.100121 & -2.387164 & 2.426775 \\
\hline $\mathrm{C}$ & 2.824802 & -1.438662 & 1.541184 \\
\hline $\mathrm{H}$ & 3.263618 & -2.408489 & 1.323853 \\
\hline $\mathrm{H}$ & 3.572105 & -0.683283 & 1.769270 \\
\hline $\mathrm{C}$ & 3.082192 & -0.949664 & -0.597929 \\
\hline $\mathrm{H}$ & 2.797655 & 0.082380 & -0.875853 \\
\hline $\mathrm{C}$ & 2.456942 & -1.978328 & -1.544439 \\
\hline $\mathrm{H}$ & 2.497754 & -2.991460 & -1.128624 \\
\hline
\end{tabular}




$\begin{array}{llll}\mathrm{H} & 1.397157 & -1.793224 & -1.790560 \\ \mathrm{H} & 2.968147 & -2.005664 & -2.517932 \\ \mathrm{C} & 1.048239 & -0.276171 & 2.935847 \\ \mathrm{C} & 1.552140 & 1.041387 & 2.737076 \\ \mathrm{C} & -0.128838 & -0.393230 & 3.727920 \\ \mathrm{C} & 0.949704 & 2.150639 & 3.343161 \\ \mathrm{H} & 2.471225 & 1.188768 & 2.172723 \\ \mathrm{C} & -0.716031 & 0.715241 & 4.323434 \\ \mathrm{H} & -0.551427 & -1.382059 & 3.891769 \\ \mathrm{C} & -0.181587 & 1.999804 & 4.141241 \\ \mathrm{H} & 1.376320 & 3.137174 & 3.182796 \\ \mathrm{H} & -1.602211 & 0.580379 & 4.938277 \\ \mathrm{H} & -0.646827 & 2.861932 & 4.608766\end{array}$




\section{References}

[S1] Gans-Eichler, T.; Gudat, D.; Nättinen, K.; Nieger, M. Chem. Eur. J. 2006, 12, 1162.

[S2] Hitchcock, P. B.; Khvostov, A. V.; Lappert, M. F.; Prochenko, A. V. J. Organomet. Chem. 2002, 647, 198.

[S3] Fu, P.-F.; Brard, L.; Li, Y.; Marks, T. J. J. Am. Chem. Soc. 1995, 117, 7157.

[S4] Konkol, M.; Kondracka, M.; Voth, P.; Spaniol, T. P.; Okuda, J. Organometallics 2008, 27, 3774.

[S5] Shirakawa, E.; Ikeda, D.; Masui, S.; Yoshida, M.; Hayashi, T. J. Am. Chem. Soc. 2011, 134, 272.

[S6] Molander, G. A.; Julius, M. J. Org. Chem. 1992, 57, 6347.

[S7] Sheldrick, G. M. Acta Crystallogr.2008, A64, 112.

[S8] Farrugia, L. J. J. Appl. Crystallogr.1997, 30, 837.

[S9] (a) Becke, A. D. J. Chem. Phys. 1993, 98, 5648; (b) Lee, C.; Yang, W.; Parr, R. G. Phys. Rev. B 1988, 37, 785; (c) Becke, A. D. J. Chem. Phys. 1993, 98, 1372.

[S10] (a) Weigand, A.; Cao, X.; Yang, J.; Dolg, M. Theor. Chem. Acc.2010, 126, 117; (b) Georgieva, L.; Trendafilova, N.; Aquino, A. J. A.; Lischka, H. Inorg. Chem. 2007, 46, 10926. (c) Yang, J.; Dolg, M. Theor. Chem. Acc. 2005, 113, 212. (d) Wang, D.; Zhao, C.; Phillips, D. L. Organometallics 2004, 23, 1953. (e) Labouille, S.; Nief, F.; Le Goff, X.-F.; Maron, L.; Kindra, D. R.; Houghton, H. L.; Ziller, J. W.; Evans, W. J. Organometallics 2012, 31, 5196.

[S11] Fukui, K. Acc. Chem. Res. 1981, 14, 363.

[S12] Zhao, Y.; Truhlar, D. G. J .Chem. Phys. 2006, 125, 194101.

[S13] Marenich, A. V.; Cramer, C. J.; Truhlar, D. G. J. Phys. Chem. B 2009, 113, 6378.

[S14] Frisch, M. J.; et al. Gaussian 09, revision E.01; Gaussian, Inc.: Wallingford, CT, 2010. 

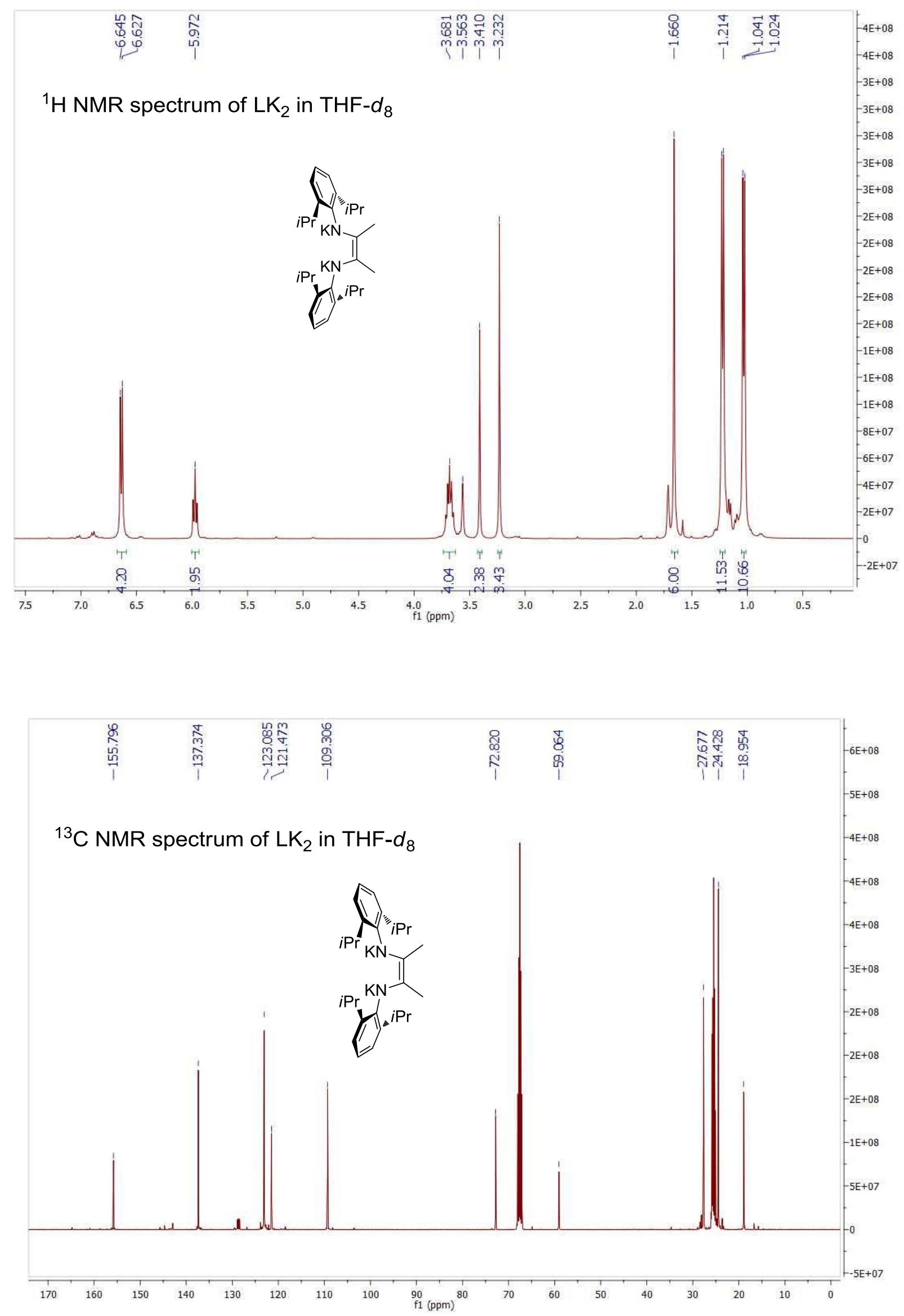

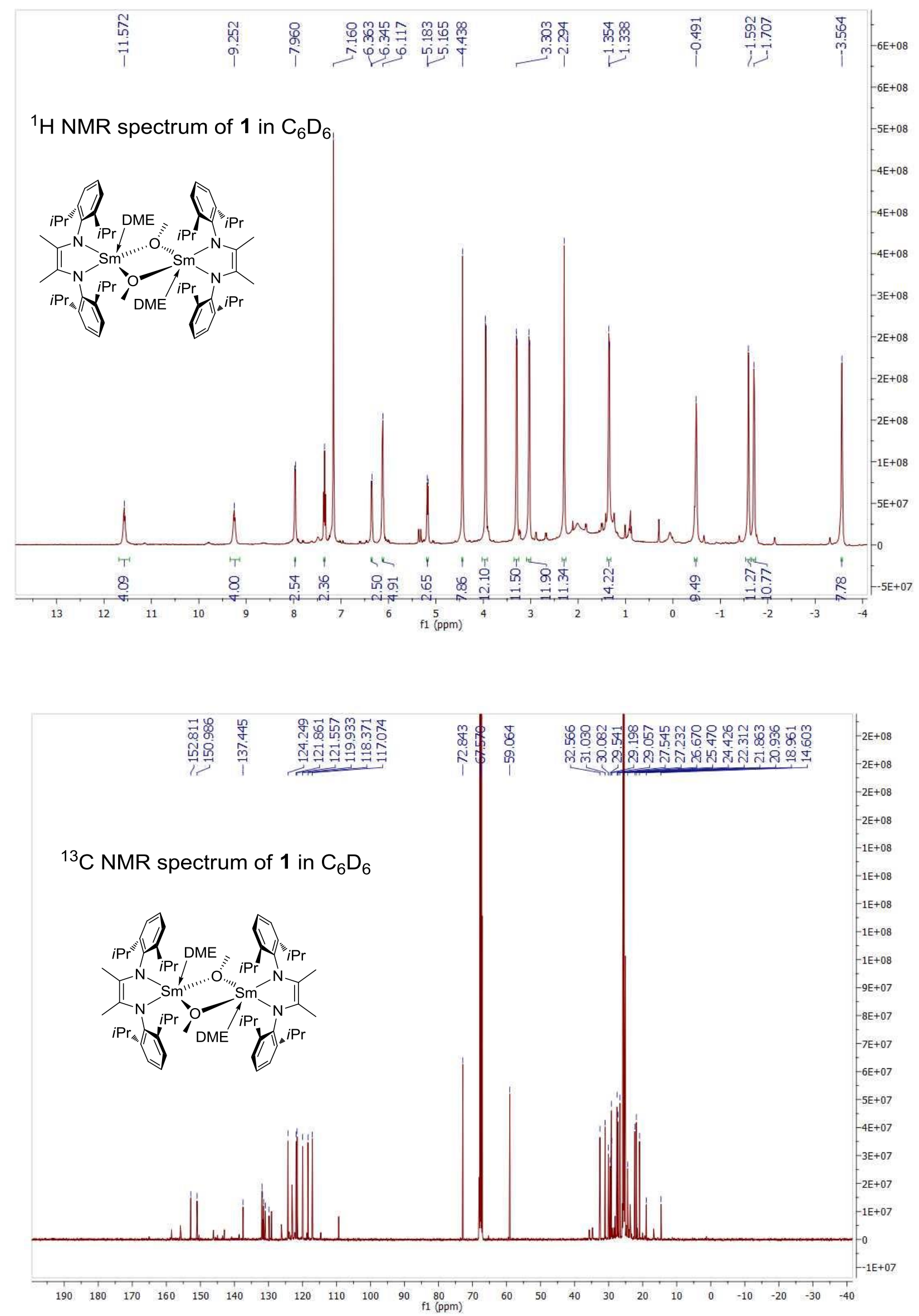

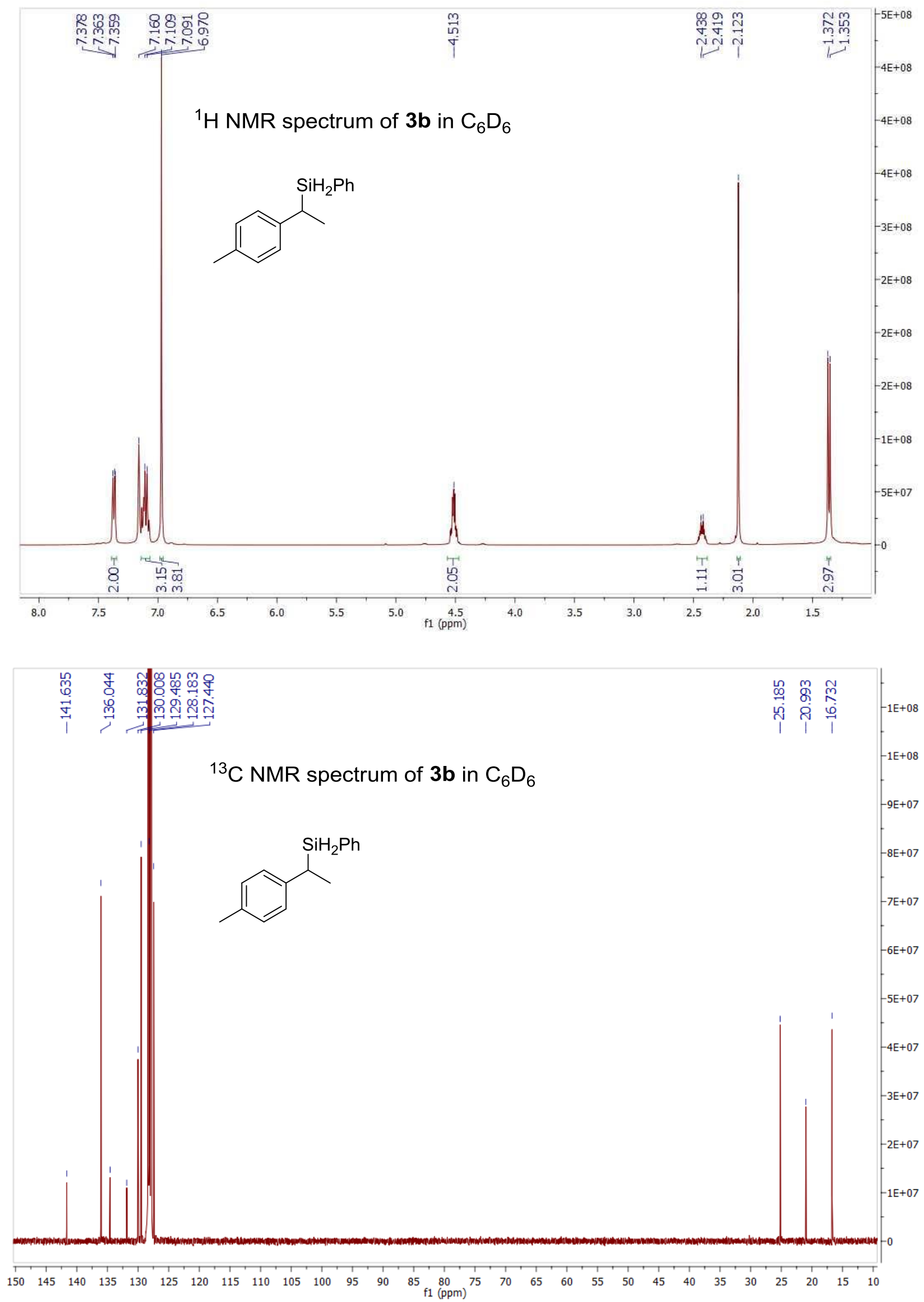


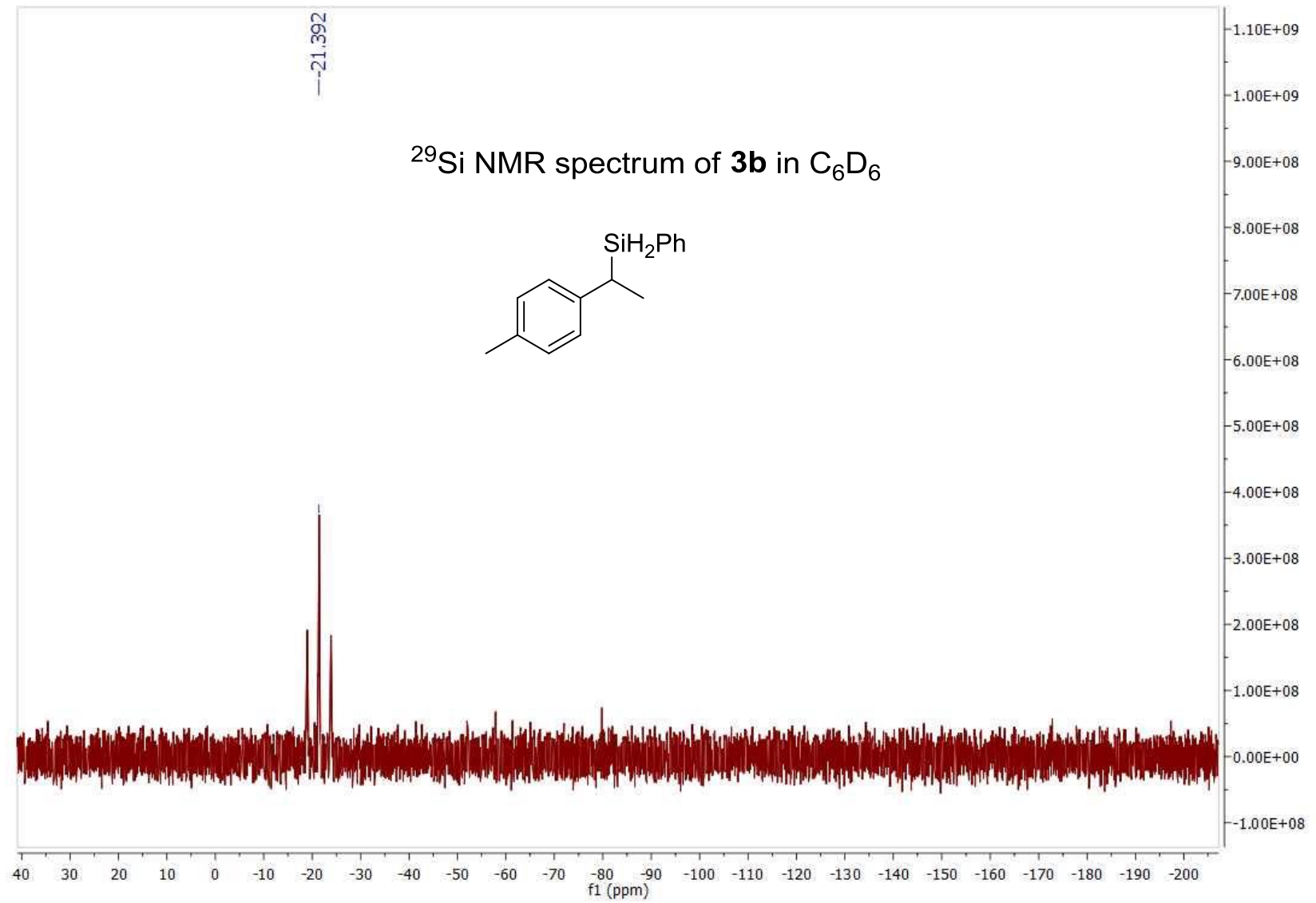



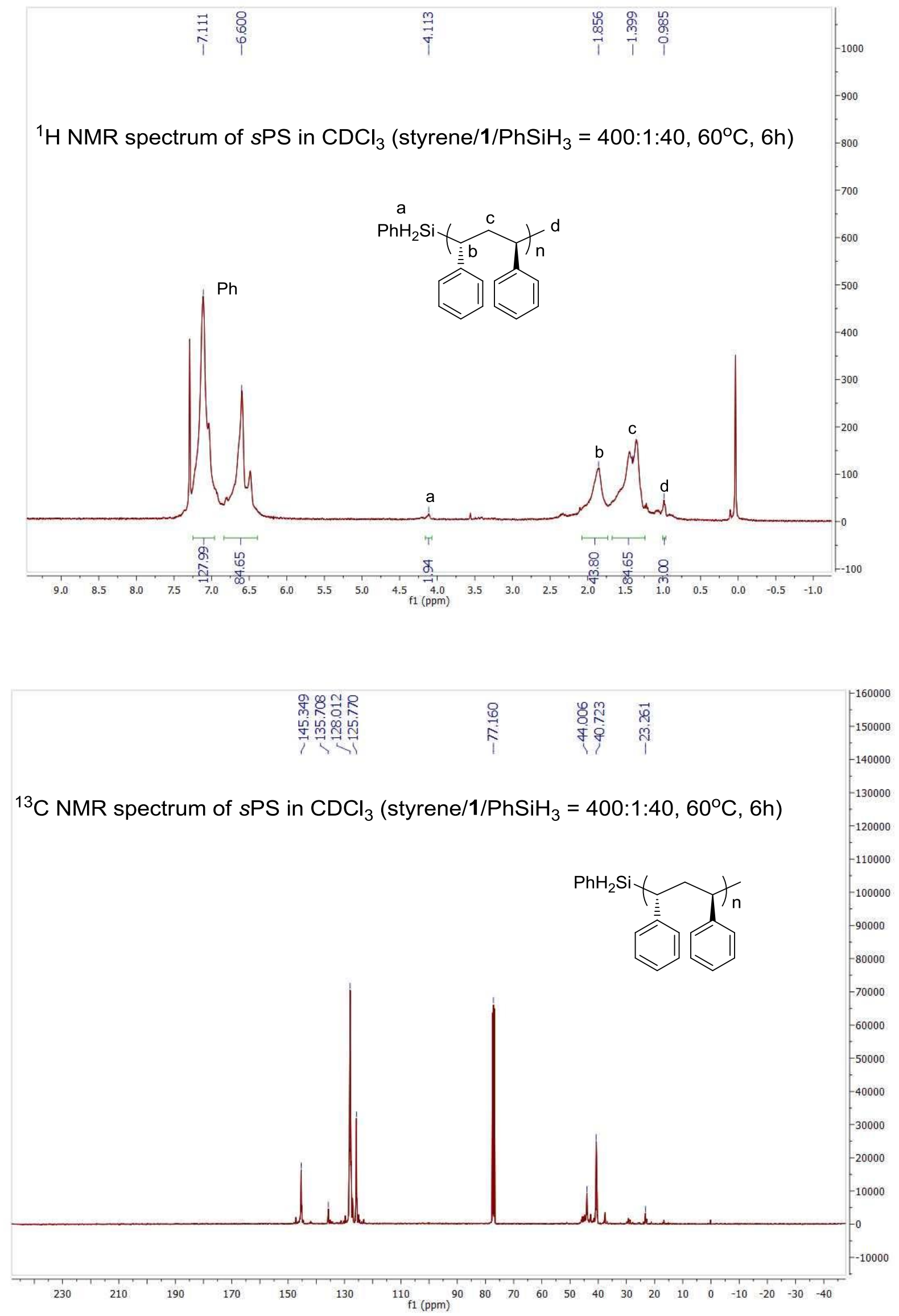

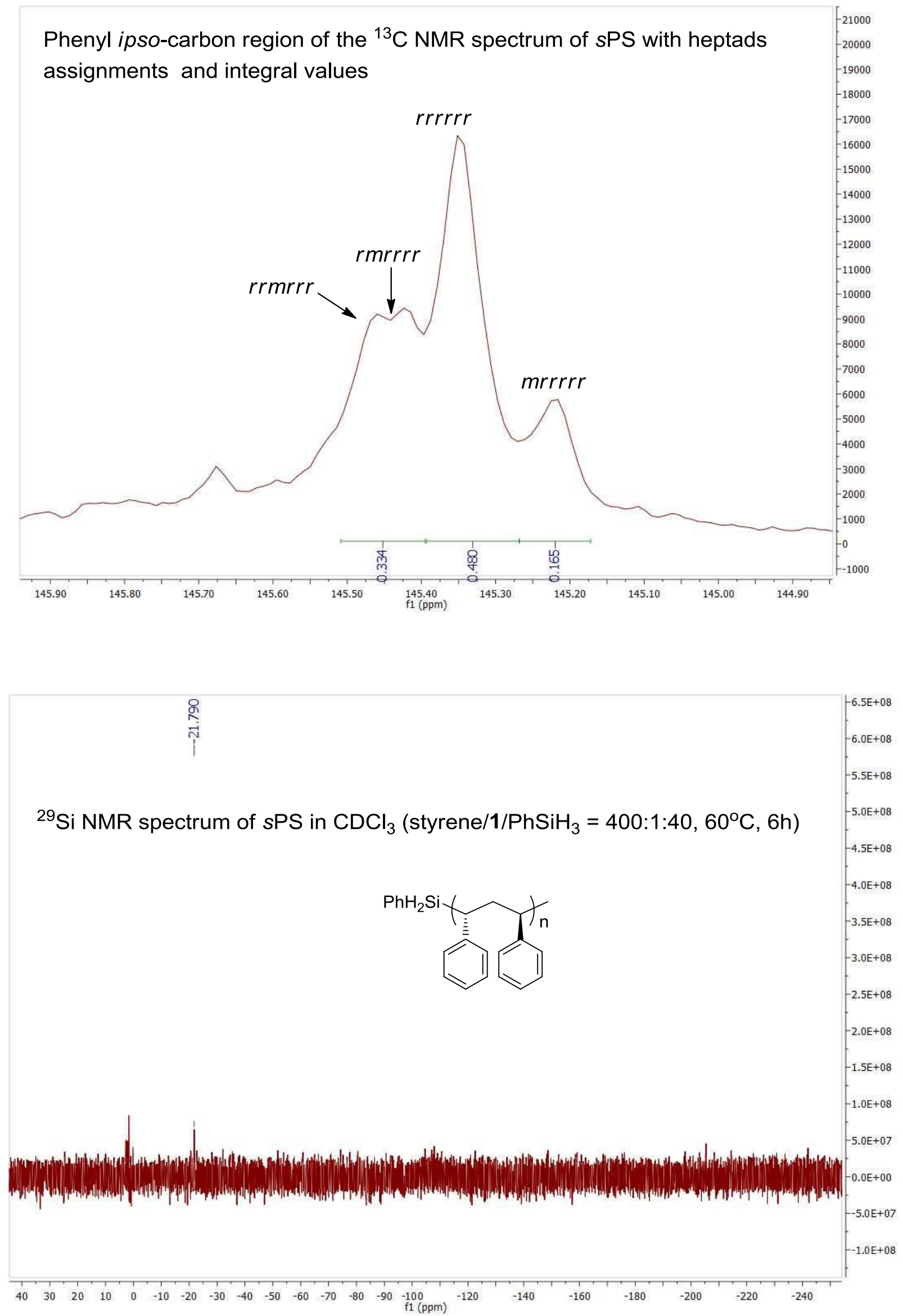

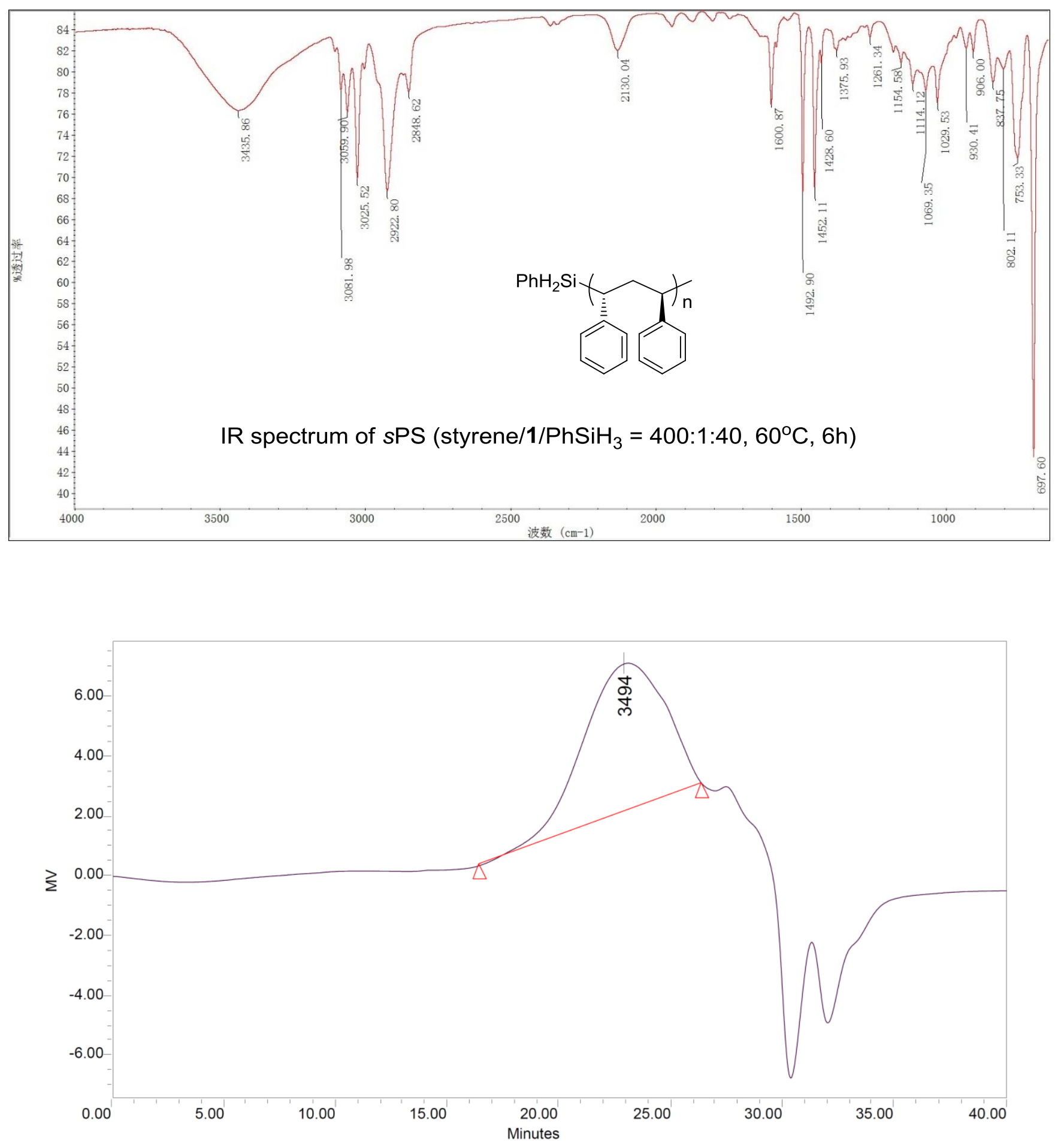

GPC Results

\begin{tabular}{|l|l|c|c|c|c|c|c|c|c|c|}
\hline Dist Name & $\begin{array}{c}\text { Elution } \\
\text { Volume } \\
(\mathrm{ml})\end{array}$ & $\begin{array}{c}\text { Retention } \\
\text { Time } \\
(\mathrm{min})\end{array}$ & $\begin{array}{c}\text { Adjusted } \\
\mathrm{RT} \\
(\mathrm{min})\end{array}$ & $\mathrm{Mn}$ & $\mathrm{Mw}$ & $\mathrm{MP}$ & $\mathrm{Mz}$ & $\mathrm{Mz}+1$ & $\mathrm{Mz} / \mathrm{Mw}$ \\
\hline 1 & & 22.933 & 22.933 & 22.933 & 5140 & 6884 & 3494 & 11651 & 21663 & 1.692635 \\
\hline
\end{tabular}



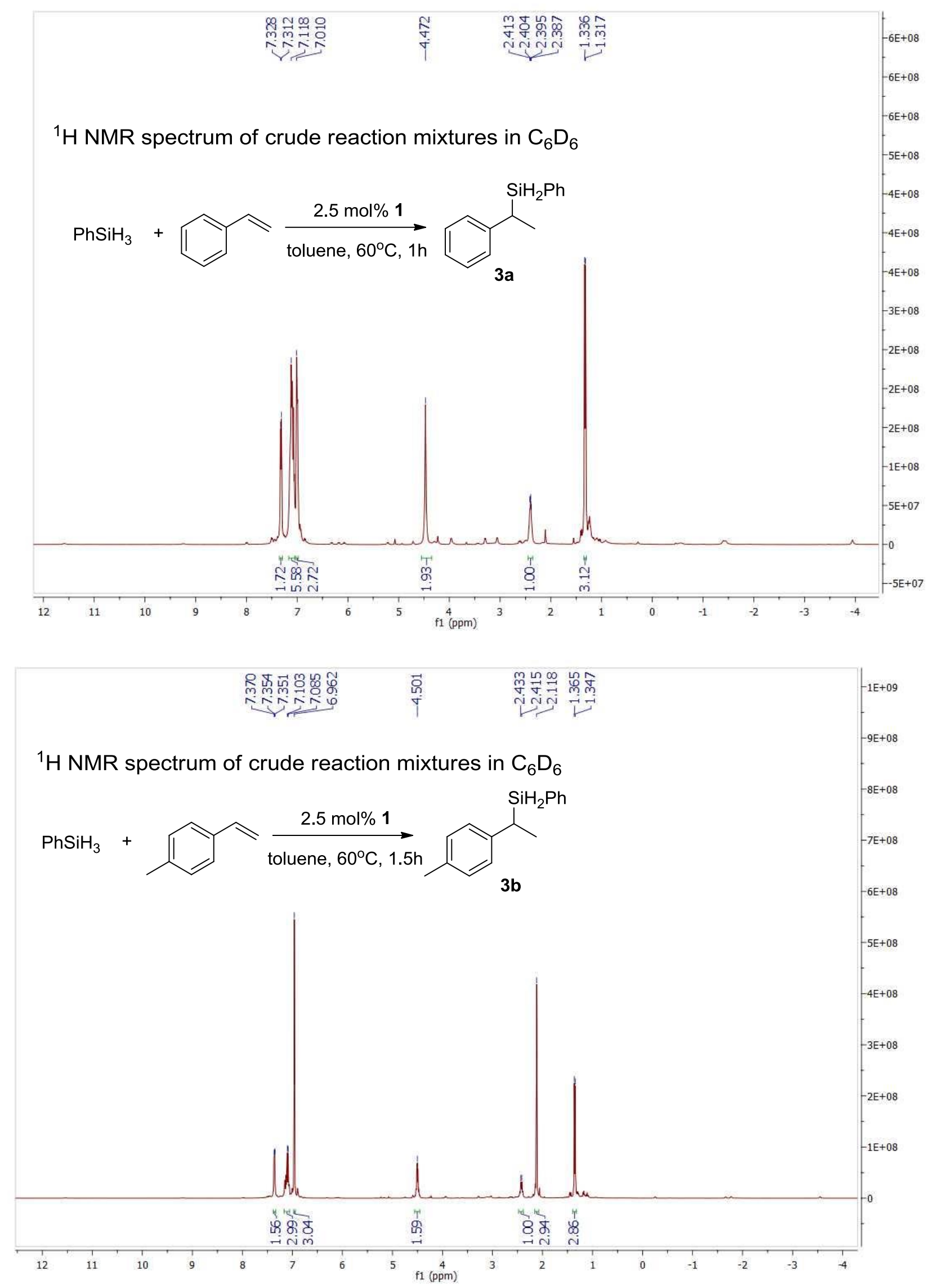


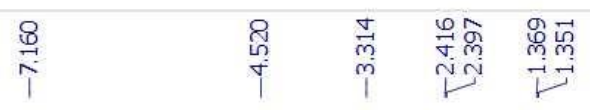

${ }^{1} \mathrm{H}$ NMR spectrum of crude reaction mixtures in $\mathrm{C}_{6} \mathrm{D}_{6}$
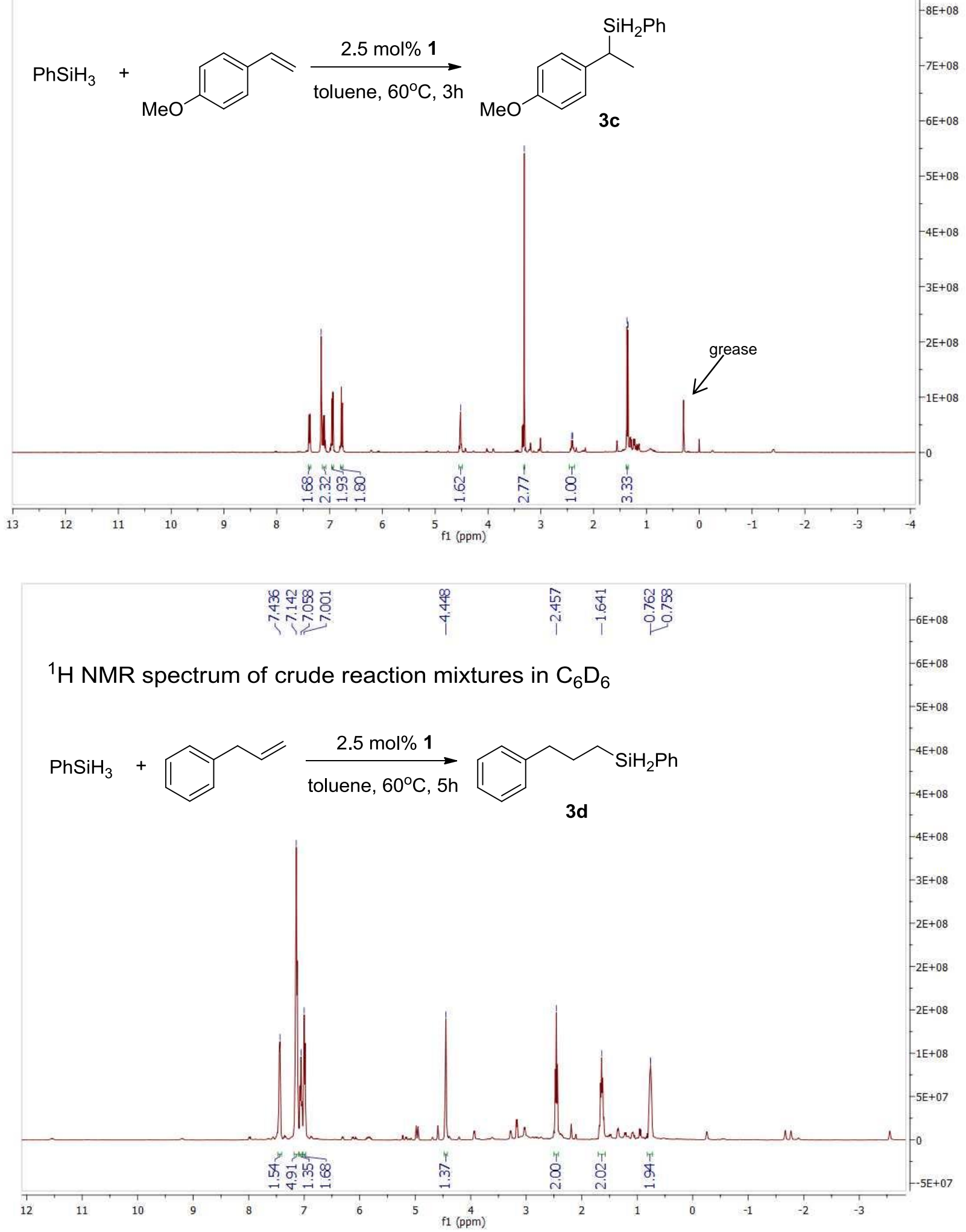


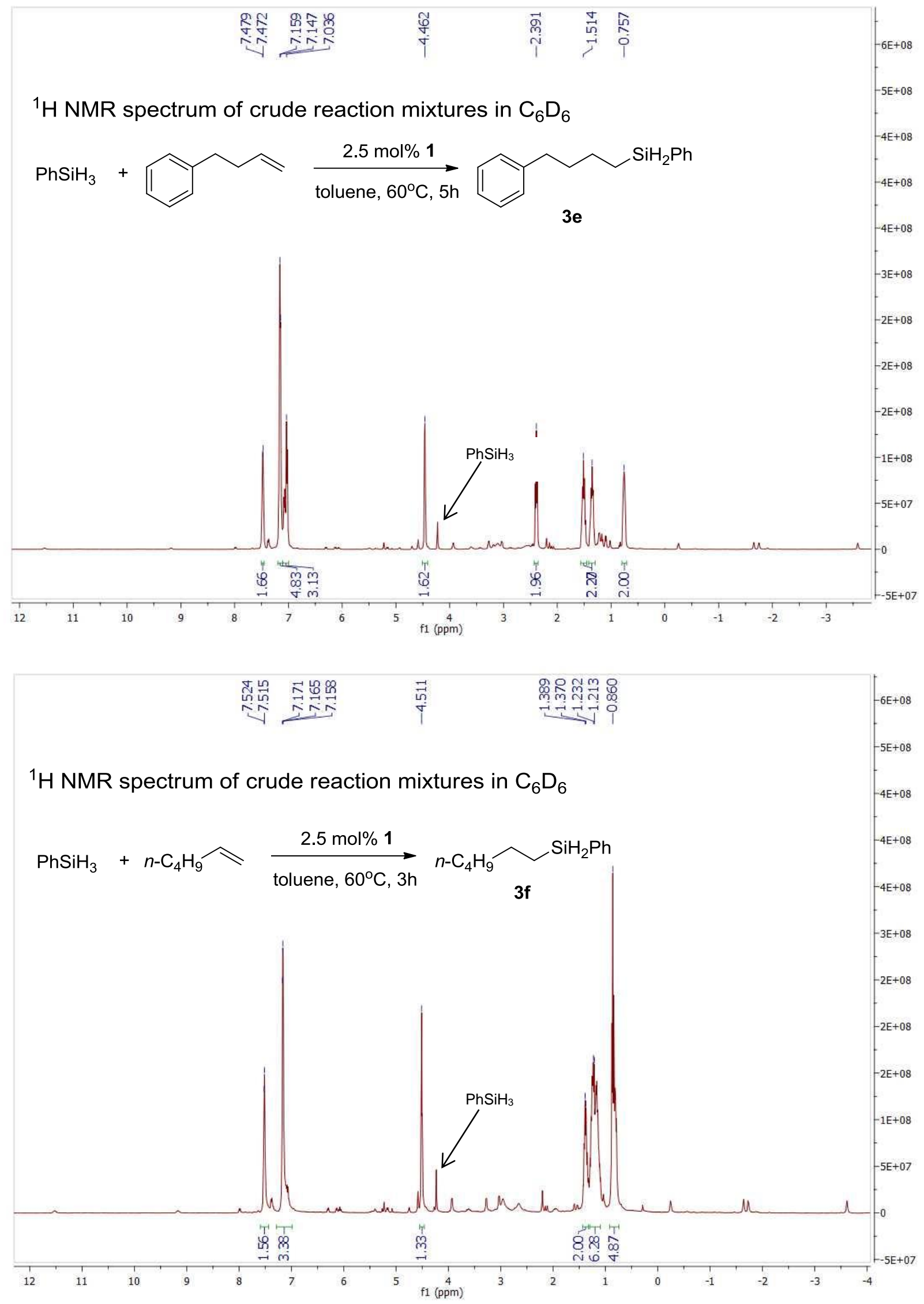




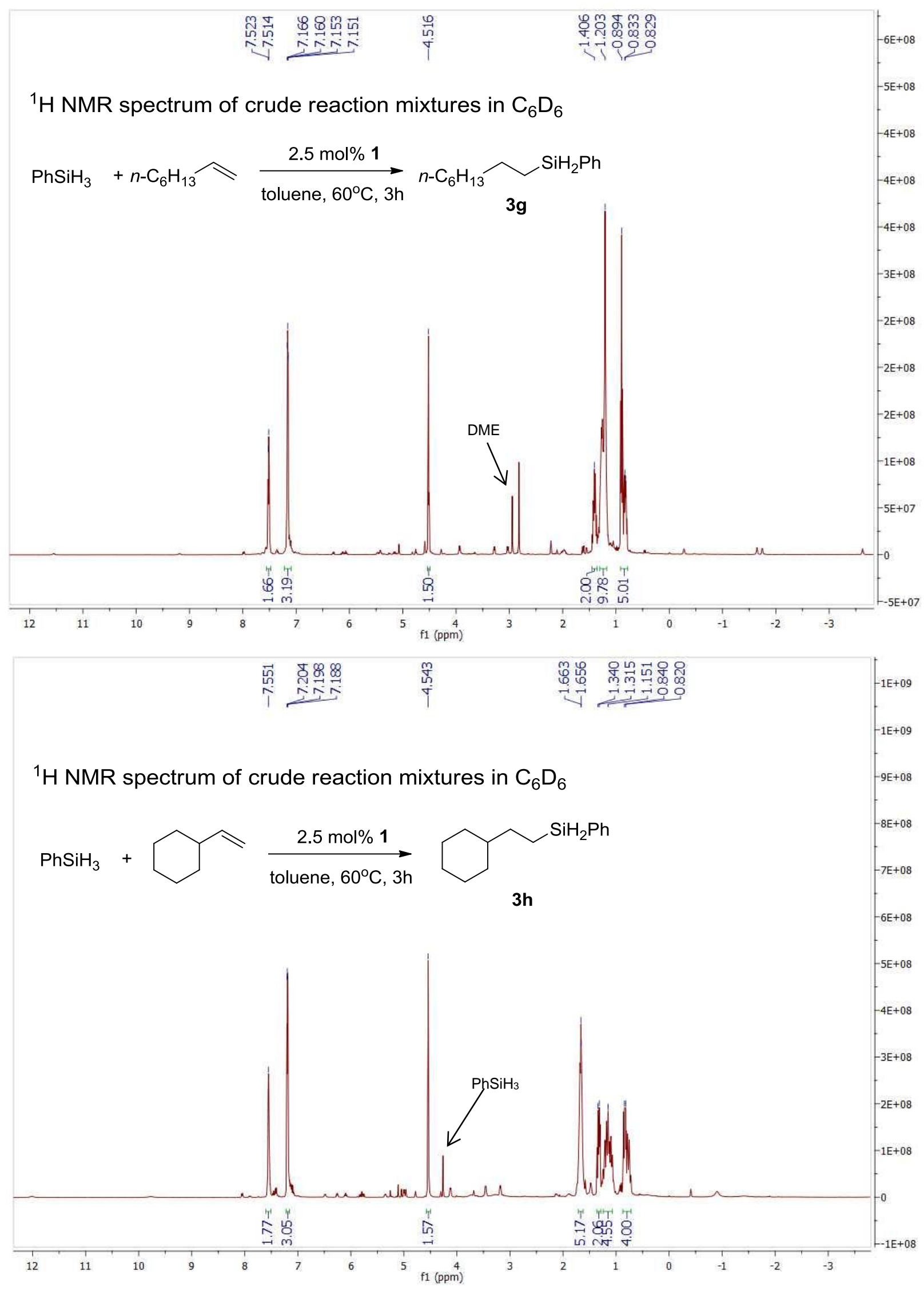




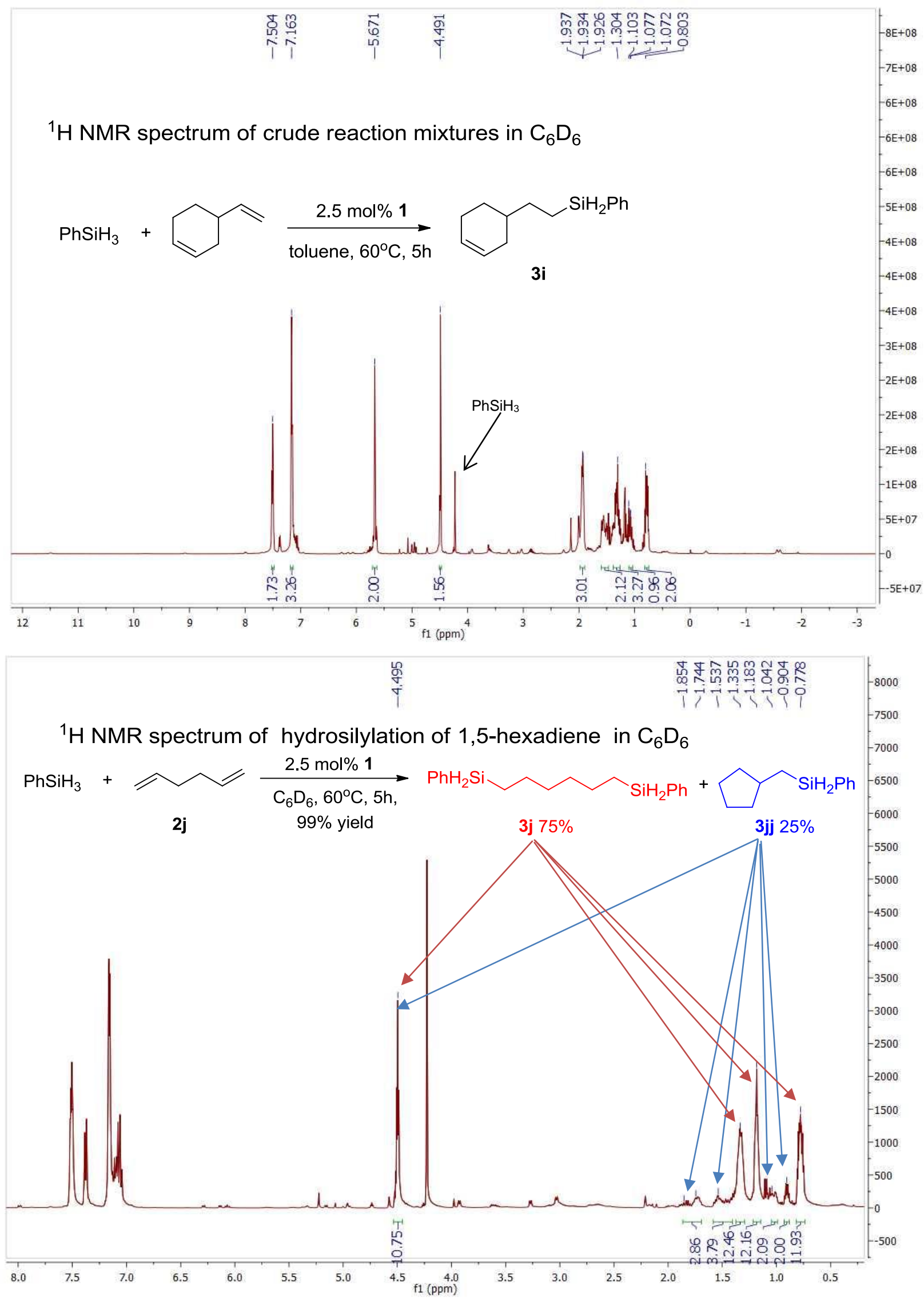


GC-MS spectrum of crude reaction mixtures in $n$-hexane

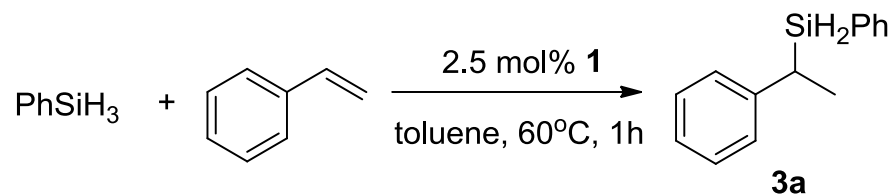

C:IDATEVlijianfenglprintlhaolvff-Ph

$11 / 25 / 14$ 15:55:37

RT: $3.51-14.25$

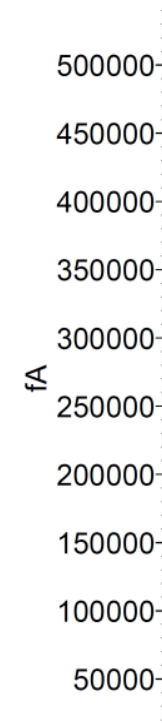

RT: 9.83

$\mathrm{NL}:$

MA: 1035880

41E5

FID

Analog

lif-Ph

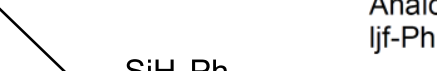


GC-MS spectrum of crude reaction mixtures in $n$-hexane

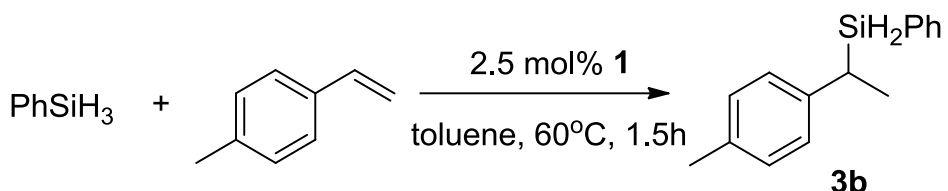

RT: 3.12 - 14.62

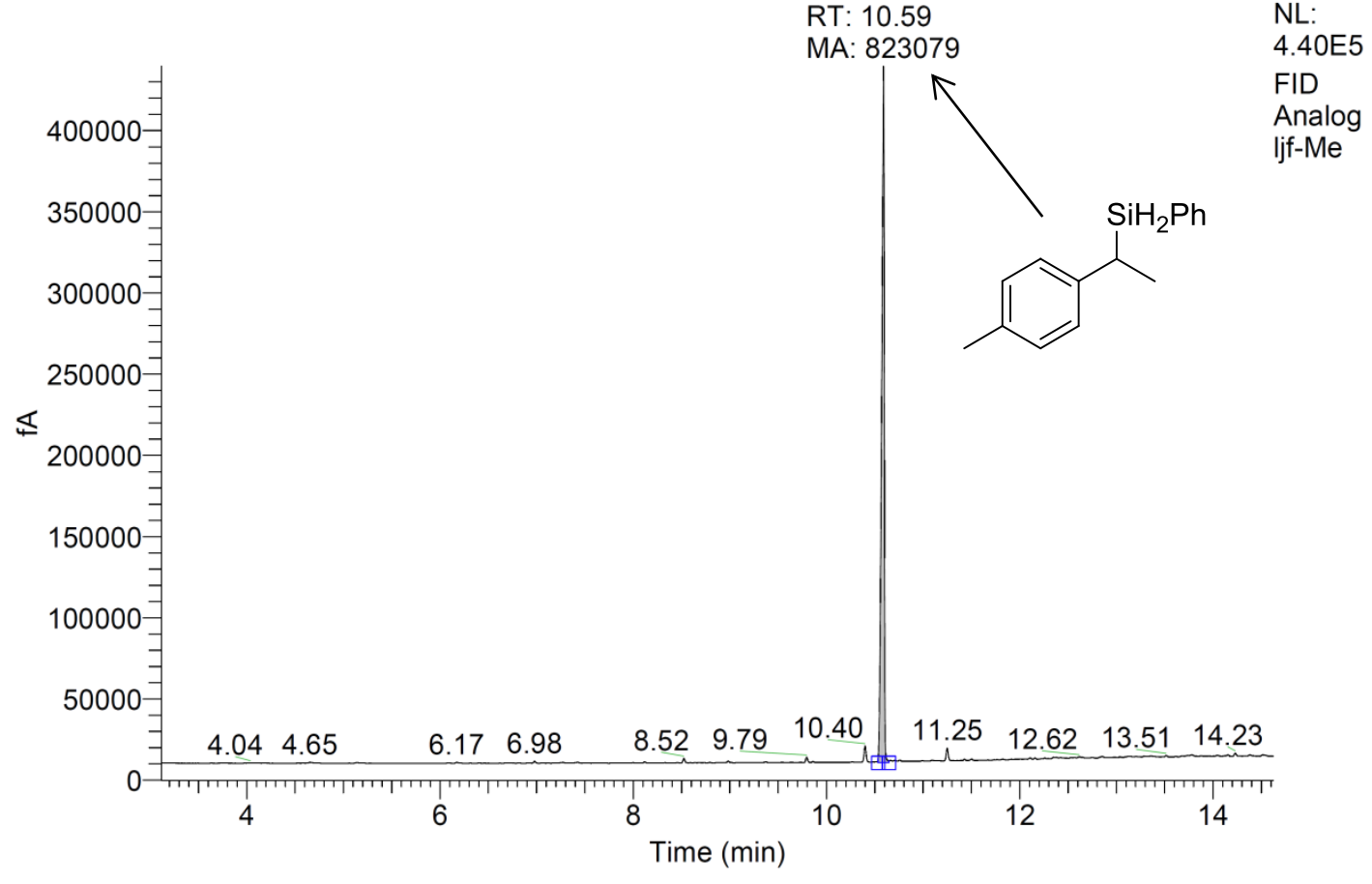


GC-MS spectrum of crude reaction mixtures in $n$-hexane<smiles>COc1ccc(C=CC=CC(C)C(C)c2ccccc2)cc1</smiles>

C:IDATEVlijianfeng Iprintlhaolljf-MeO

12/09/14 14:06:09

RT: $3.37-14.40$

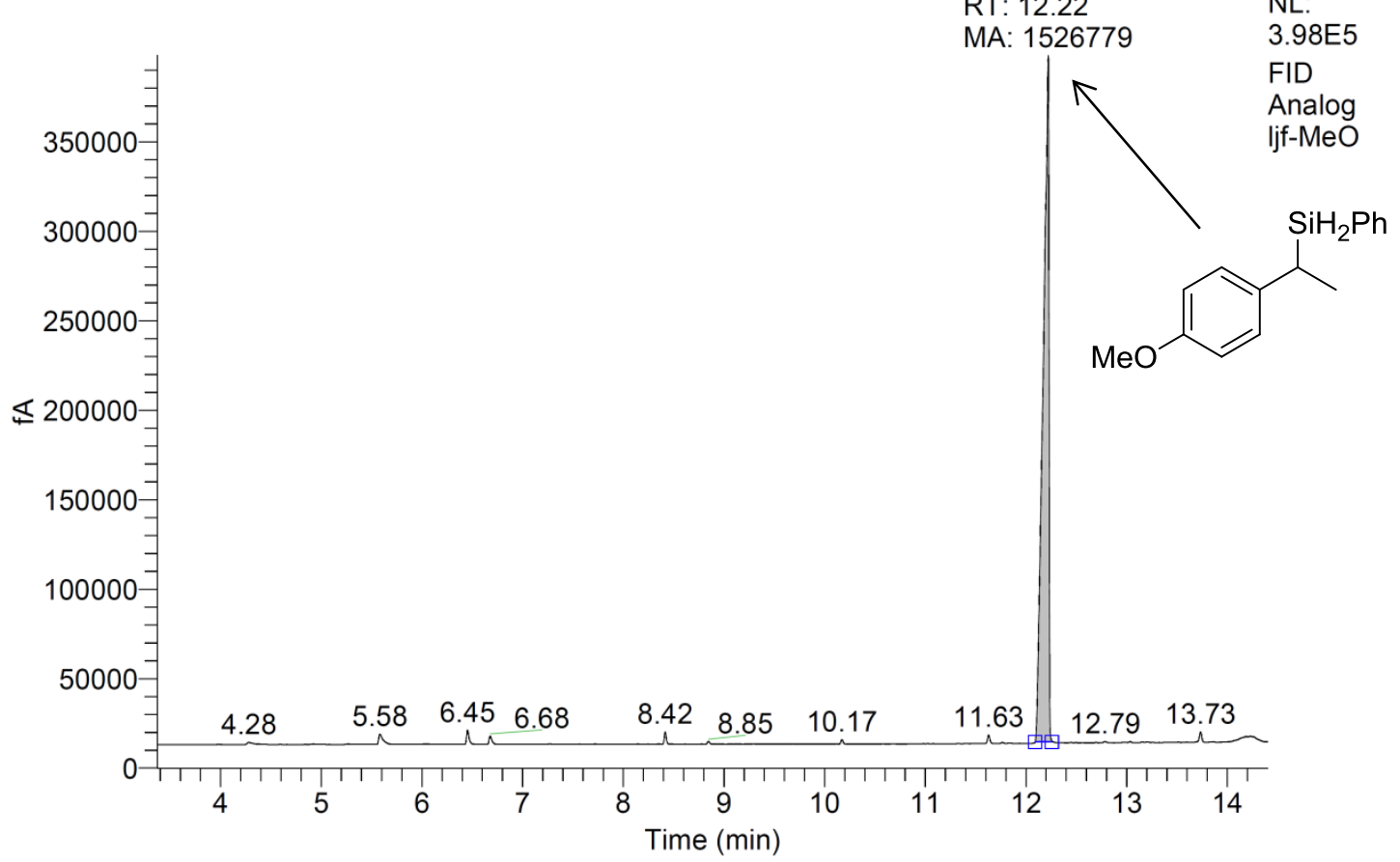


GC-MS spectrum of crude reaction mixtures in $n$-hexane<smiles>C=CCc1ccccc1</smiles>

C:IDATEVlijianfeng Iprintthaolvif-3

11/27/14 21:14:18

RT: 3.41 - 14.22

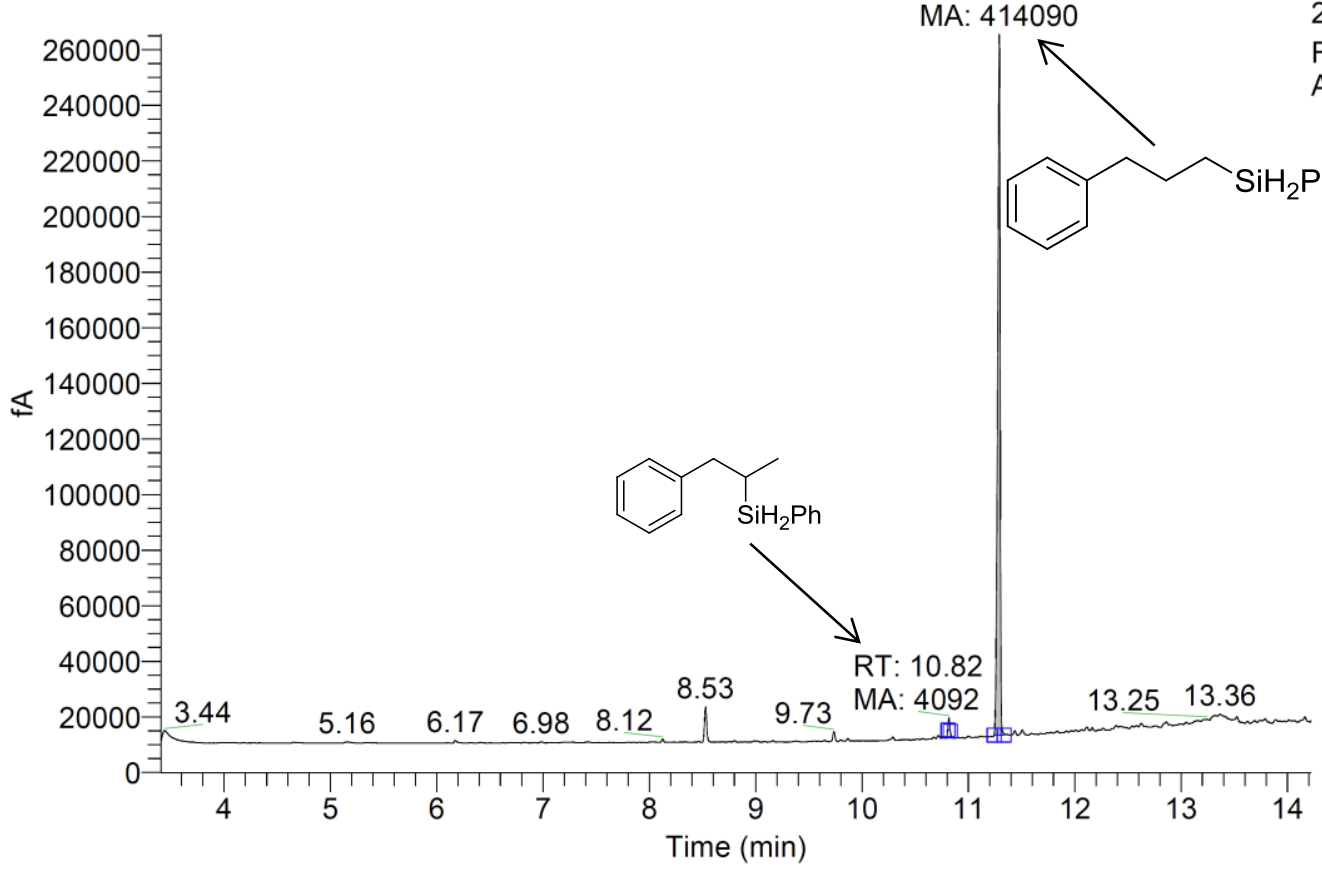

$\mathrm{NL}:$

2.65E5

FID

Analog ljf-3 
GC-MS spectrum of crude reaction mixtures in $n$-hexane<smiles>C=CCCc1ccccc1</smiles>

C:IDATEVlijianfenglprint|haolvif-4

RT: 4.80 - 14.53

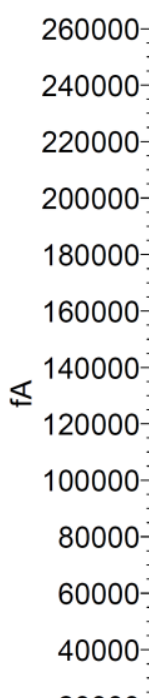

$11 / 27 / 1411: 45: 46$

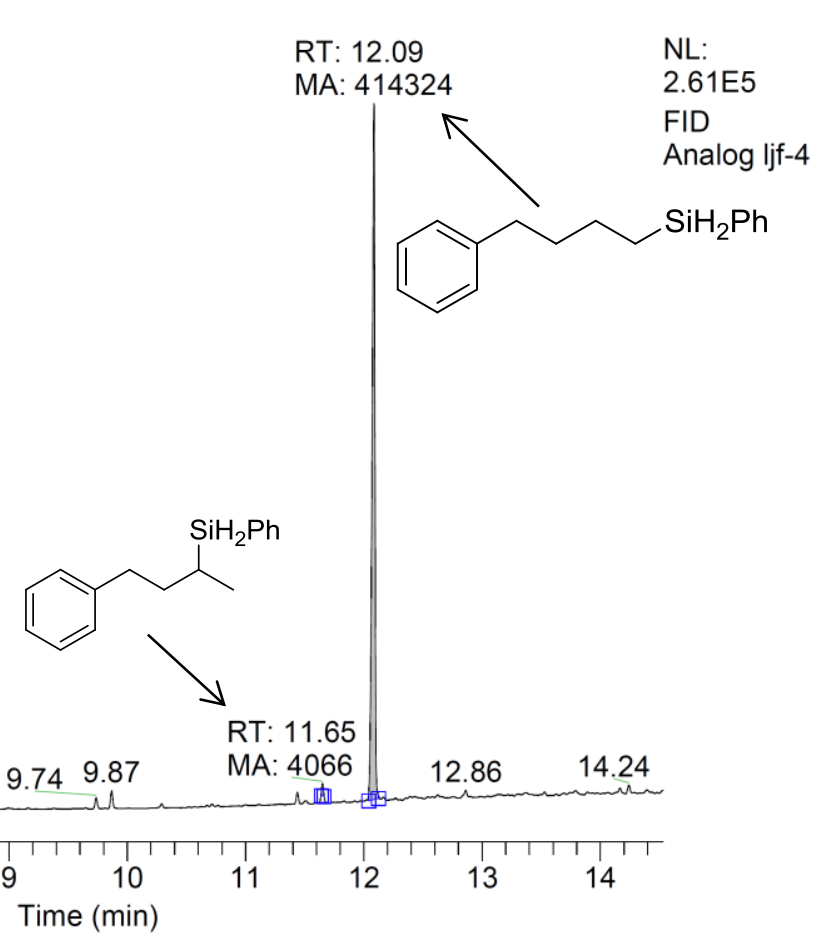


GC-MS spectrum of crude reaction mixtures in $n$-hexane

$$
\mathrm{PhSiH}_{3}+n-\mathrm{C}_{4} \mathrm{H}_{9} \curvearrowright \underset{\text { toluene, } 60^{\circ} \mathrm{C}, 3 \mathrm{~h}}{\stackrel{2.5 \mathrm{~mol} \% 1}{\longrightarrow}} n-\mathrm{C}_{4} \mathrm{H}_{9} \overbrace{\mathbf{3 f}}^{\sim} \mathrm{SiH}_{2} \mathrm{Ph}
$$

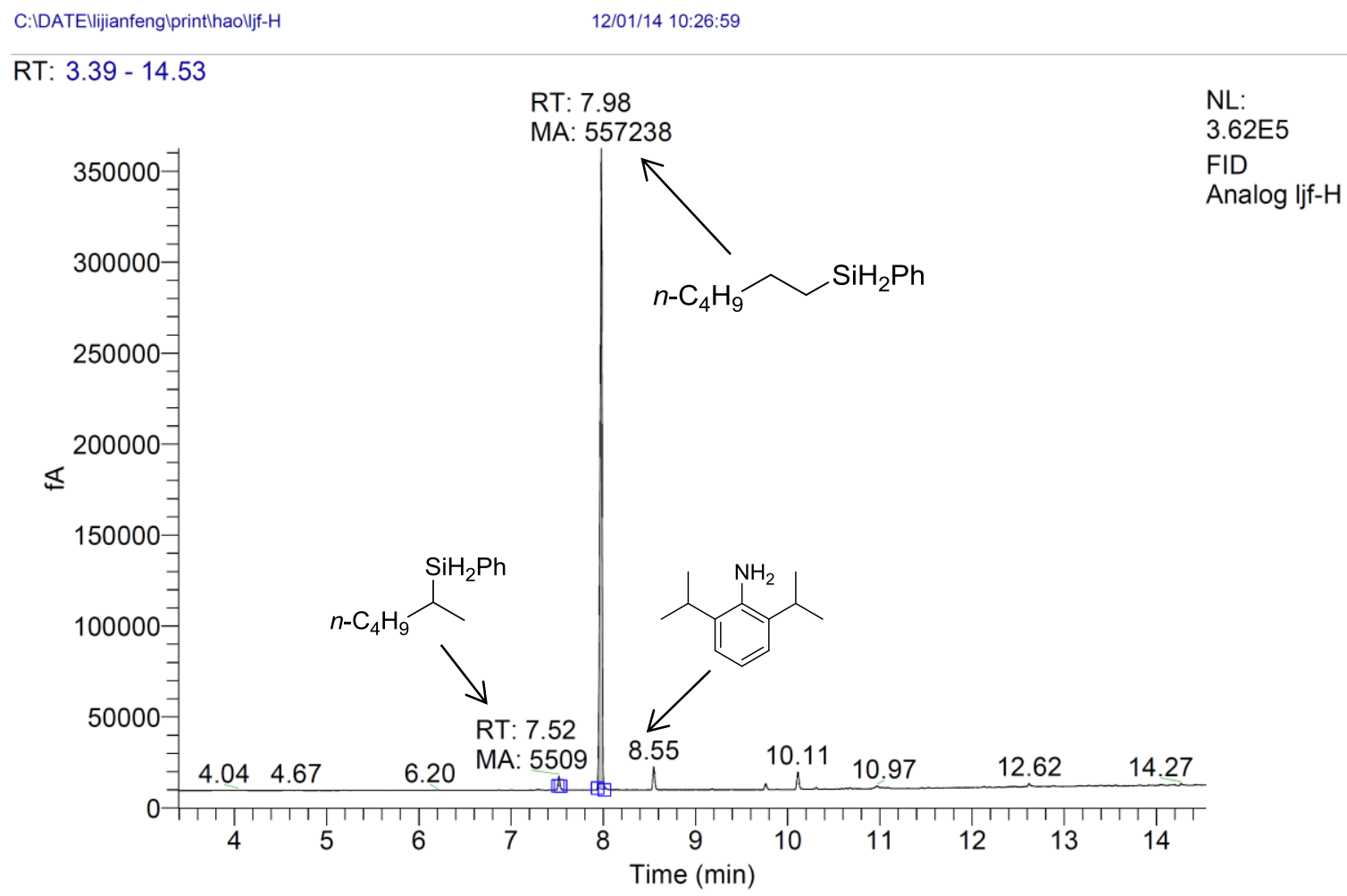


GC-MS spectrum of crude reaction mixtures in $n$-hexane

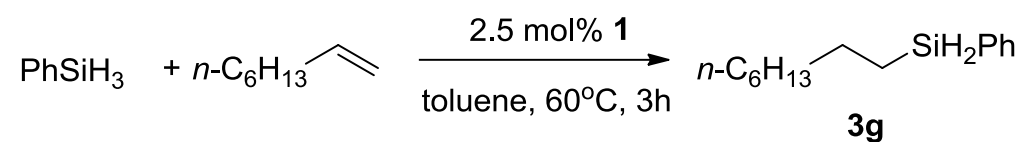

C:IDATEVlijianfeng Iprintthaolijf-O

$12 / 01 / 1410: 50: 32$

RT: 3.57 - 14.55

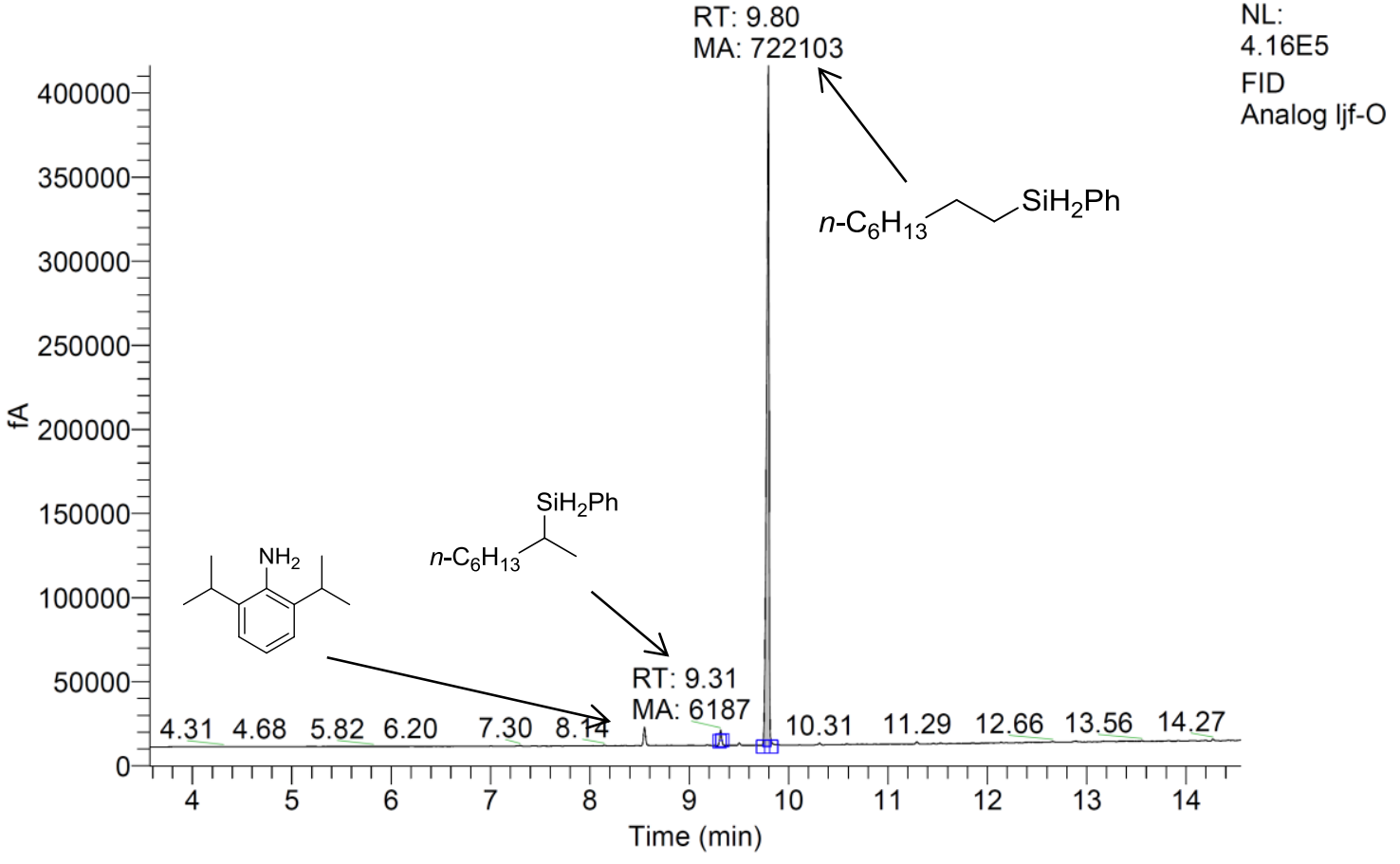


GC-MS spectrum of crude reaction mixtures in $n$-hexane

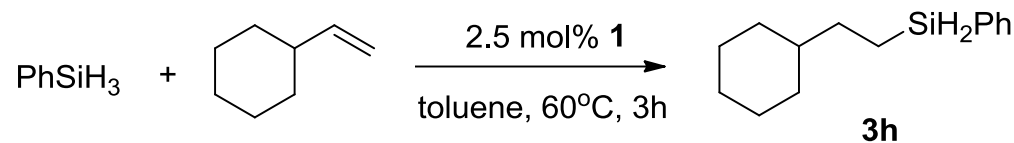

$3 h$

C:IDATEVlijianfenglprint/haolljf-Ha

11/25/14 18:39:29

RT: 3.54 - 14.37

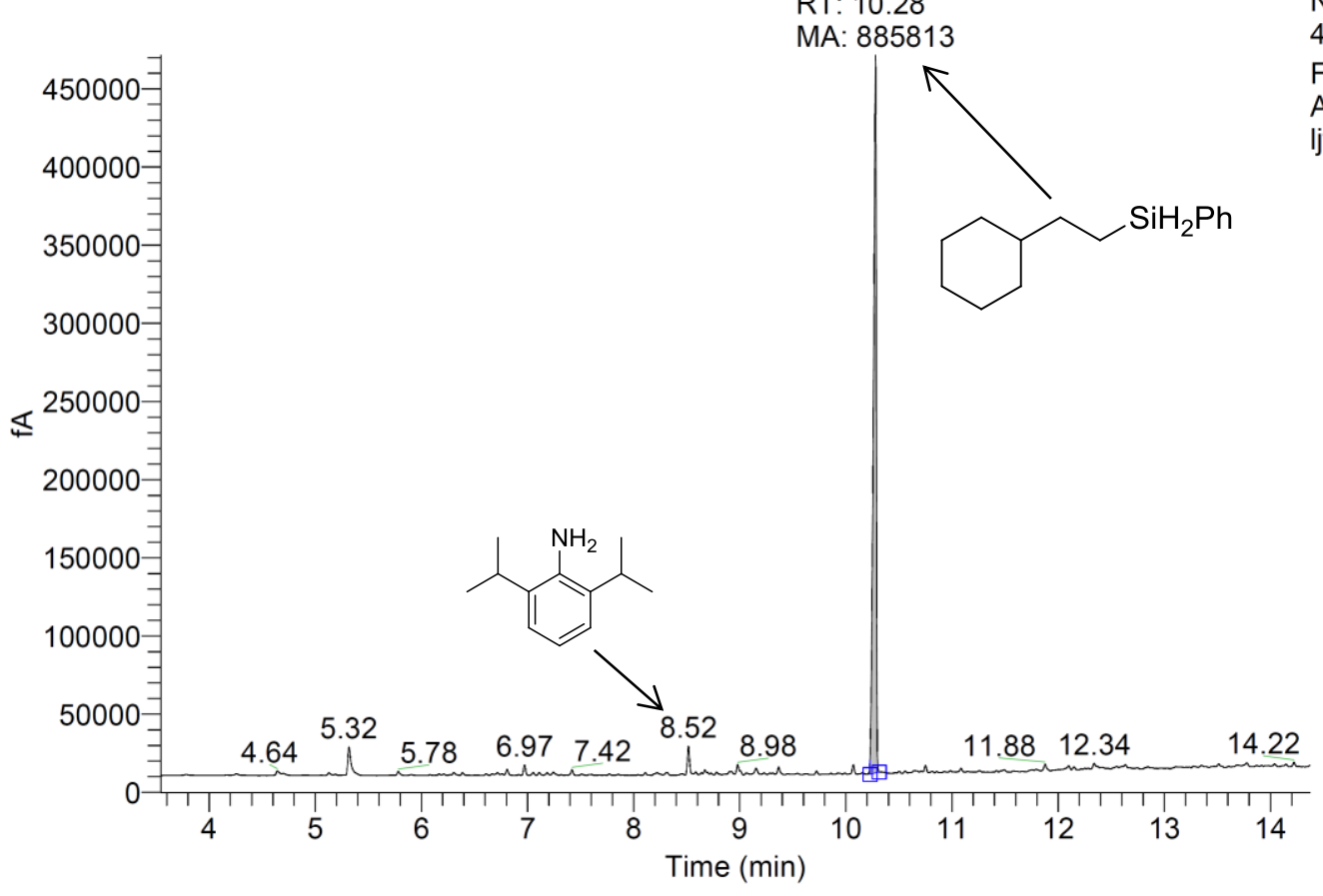

FID

Analog

ljf-Ha 
GC-MS spectrum of crude reaction mixtures in $n$-hexane<smiles>[Pb]=CC=CC=CC1CC=CCC1</smiles>

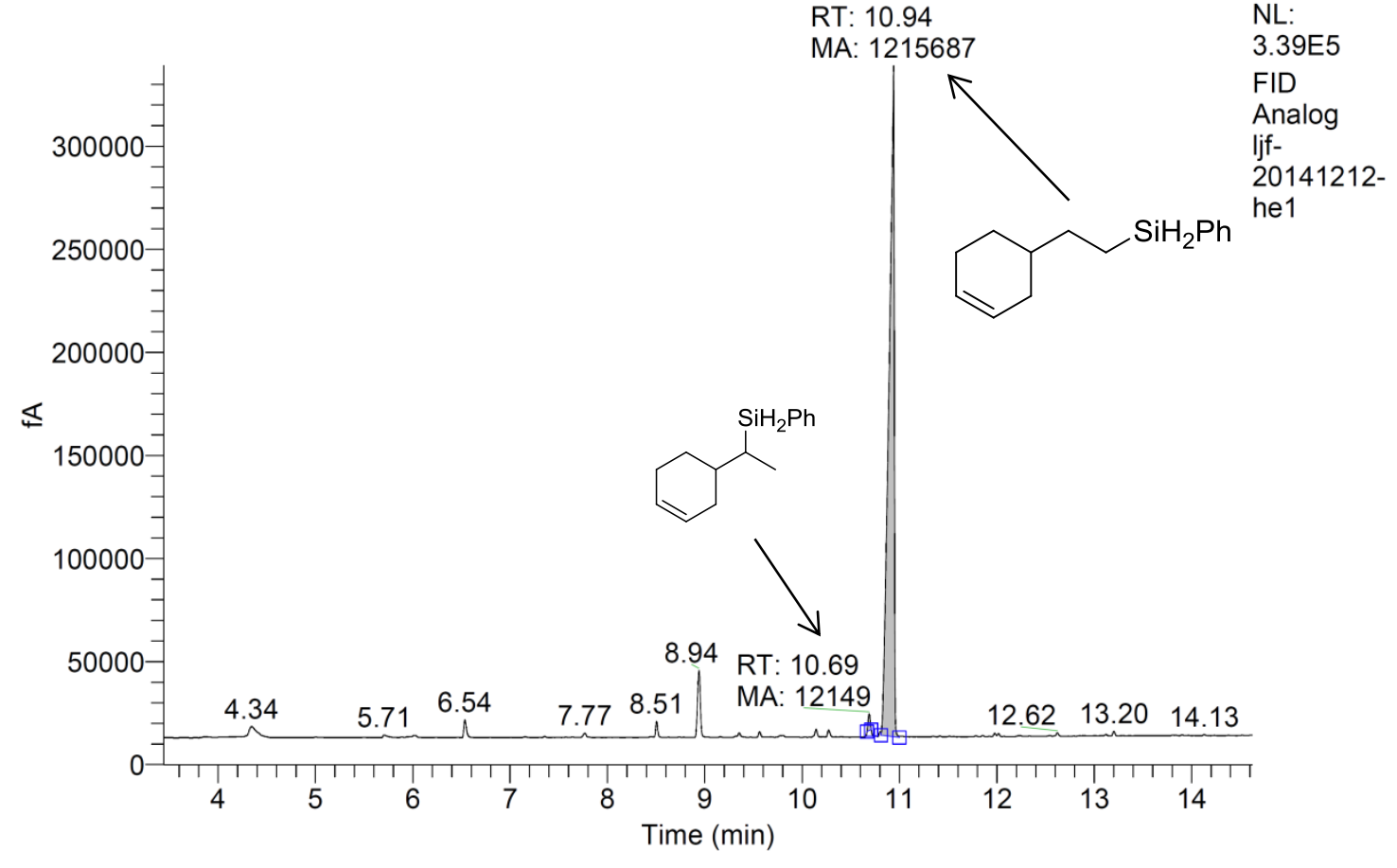

